\title{
THE COMBINATORICS OF QUIVER REPRESENTATIONS
}

\author{
HARM DERKSEN AND JERZY WEYMAN
}

\section{CONTENTs}

1. Introduction

1.1. Main results

1.2. Horn's conjecture and related problems

1.3. The quiver method

1.4. Organization

2. Preliminaries

2.1. Basic notions for quivers

2.2. Semi-invariants for quiver representations

2.3. Representations in general position

2.4. The canonical decomposition

2.5. The combinatorics of dimension vectors

2.6. Perpendicular categories

2.7. Exceptional Sequences

2.8. Stability and GIT-quotients

3. Stability

3.1. Harder-Narasimhan and Jordan-Hölder filtrations

3.2. The $\sigma$-stable decomposition

4. Schur sequences

5. The faces of the cone $\mathbb{R}_{+} \Sigma(Q, \alpha)$

6 . More on the $\sigma$-stable decompositions

$\frac{1}{1}$

6.1. The set of $\sigma$-stable dimension vectors

6.2. $\sigma$-stable decomposition for quivers with oriented cycles

7. Littlewood-Richardson coefficients

7.1. The Klyachko cone

7.3. Faces of the Klyachko cone of arbitrary codimension

7.4. A multiplicative formula for Littlewood-Richardson coefficients 46

Appendix: Belkale's proof of Fulton's conjecture 48

References

\section{INTRODUCTION}

1.1. Main results. Let $\alpha, \beta$ be dimension vectors for a quiver $Q$ without oriented cycles. If $\langle\alpha, \beta\rangle_{Q}=$ 0 , where $\langle\cdot, \cdot\rangle_{Q}$ is the Euler form (or Ringel form), then we will define a number $\alpha \circ \beta$. This number

The first author was supported by NSF, grant DMS 0349019 and the second author was supported by NSF, grant DMS 0300064. 
can be defined as the dimension of a space $\operatorname{SI}(Q, \beta)_{\langle\alpha, \cdot\rangle}$ of semi-invariants (see Definition 2.5). It was shown in [16] that $\alpha \circ \beta$ can also be defined in terms of Schubert calculus. In the Schubert calculus approach, $\alpha \circ \beta$ counts the number of $\alpha$-dimensional subrepresentations of a general $(\alpha+\beta)$ dimensional representation. In the special case of the triple flag quiver, the number $\alpha \circ \beta$ turns out to a be Littlewood-Richardson coefficient $c_{\lambda, \mu}^{\nu}$ where the partitions $\lambda(\alpha, \beta), \mu=\mu(\alpha, \beta), \nu=\nu(\alpha, \beta)$ depend on $\alpha$ and $\beta$. This allows us to prove many new results about LR-coefficients, and to extend results about Littlewood-Richardson coefficients to the more general setting of quiver representations.

Knutson and Tao (see [28]) proved the saturation conjecture for LR-coefficients: if $c_{N \lambda, N \mu}^{N \nu}>0$ for some positive integer $N$, then $c_{\lambda, \mu}^{\nu}>0$ (see Theorem 1.4). It was shown in 13] that the saturation theorem for LR-coefficients generalizes to quivers: if $N \alpha \circ M \beta>0$ for some positive integers $M, N$, then $\alpha \circ \beta>0$. Fulton conjectured that $c_{\lambda, \mu}^{\nu}=1$ implies that $c_{N \lambda, N \mu}^{N \nu}=1$ for all positive integers $N$. Knutson, Tao and Woodward proved this conjecture in [29] (see Theorem 1.6). Belkale gave a geometric proof of this result (see [4]). Belkale's proof generalizes to the quiver setting; we show that if $\alpha \circ \beta=1$, then $N \alpha \circ M \beta=1$ for all positive integers $N, M$. A proof, following Belkale, is in the Appendix. The description of Knutson, Tao and Woodward of the walls of the Klyachko cone in [29] (see Theorem 1.5) generalizes to the quiver setting as well. In this paper, we extend this result by giving a combinatorial description of the faces of the cone of arbitrary dimension (Theorem 5.1 and Corollary 7.10). We also prove that, if a triple of partitions $(\lambda, \mu, \nu)$ lies on a wall of the Klyachko cone, then the LR-coefficient $c_{\lambda, \mu}^{\nu}$ is a product of smaller LR-coefficients (Theorem 7.14).

The main technical tool we introduce in this paper is the notion of Schur sequences. A Schur sequence is a sequence of Schur roots $\alpha_{1}, \ldots, \alpha_{s}$ such that $\alpha_{i} \circ \alpha_{j}=1$ for all $i<j$. Schur sequences are a natural generalizations of exceptional sequences, allowing imaginary Schur roots to appear instead of only real Schur roots. Schur sequences occur naturally as the dimension vectors appearing in the canonical decomposition of a dimension vector. In this paper we also study the dimension vectors of the factors in a Jordan-Hölder filtration of a $\sigma$-semi-stable representation. This leads to the notion of the $\sigma$-stable decomposition of a dimension vector. Again, the dimensions in the $\sigma$-stable decomposition form a Schur sequence. A crucial result is that every Schur sequence can be refined to an exceptional sequence.

Before proving our main results, we review various notions such as perpendicular categories, exceptional sequences and stability for quivers.

The main results of the paper have interesting applications. We use exceptional sequences to "embed" the category of representation of a quiver $Q$ into the category of representations of another quiver $Q^{\prime}$. This is sometimes possible even when $Q$ is not a subquiver $Q^{\prime}$. Using this approach we construct a triple of partitions $(\lambda, \mu, \nu)$ lying on an extremal ray of the Klyachko cone, such that $c_{\lambda, \mu}^{\nu}>1$. In [8], Calin Chindris and the authors constructed a counterexample to Okounkov's conjecture stating that LR-coefficients are log-concave functions of the partitions, using this embedding method. The Embedding Theorem was also used by the first author to give examples of small Galois groups in Schubert type problems (see 42] [2.10,5.13]).

The current paper can be viewed as a culmination of the series of papers [13, [14, 15, [16]. It provides the tools to systematically use the ideas of these papers.

1.2. Horn's conjecture and related problems. A classical topic going back to Herman Weyl 43] is to compare the eigenvalues of two Hermitian $n \times n$ matrices $A, B$ with the eigenvalues of their sum $C:=A+B$. For a Hermitian matrix with eigenvalues $\lambda_{1} \geq \lambda_{2} \geq \cdots \geq \lambda_{n}$ define $s(A)=\left(\lambda_{1}, \ldots, \lambda_{n}\right) \in$ $\mathbb{R}^{n}$. One would like to understand the set

$$
\mathcal{K}_{n}:=\left\{(s(A), s(B), s(C)) \in \mathbb{R}^{3 n} \mid A, B, C \text { Hermitian, } C=A+B\right\} .
$$

It turns out that $\mathcal{K}_{n}$ is given by the trace equation

$$
\lambda_{1}+\cdots+\lambda_{n}+\mu_{1}+\cdots+\mu_{n}=\nu_{1}+\cdots+\nu_{n}
$$


the weakly decreasing conditions

$$
\lambda_{1} \geq \cdots \geq \lambda_{n} \quad, \mu_{1} \geq \cdots \geq \mu_{n}, \quad \nu_{1} \geq \cdots \geq \nu_{n} .
$$

and finitely many inequalities of the form

$$
\sum_{i \in I} \lambda_{i}+\sum_{j \in J} \mu_{j} \geq \sum_{k \in K} \nu_{k}
$$

where $I, J, K$ are subsets of $\{1,2, \ldots, n\}$ of the same cardinality. We will denote the inequality (3) by $\left(\star_{I, J, K}\right)$. Horn made in 1962 a precise conjecture about triples $(I, J, K)$ for which the corresponding inequalities $\left(\star_{I, J, K}\right)$ define $\mathcal{K}_{n}$ (see [21]). Horn's conjecture provides a recursive procedure to determine all those triples $(I, J, K)$. This conjecture has been proved as a result of work by Klyachko, Totaro, Knutson and Tao and others. We will state here a closely related statement about the recursive nature of the inequalities defining $\mathcal{K}_{n}$. For

$$
I=\left\{i_{1}, i_{2}, \ldots, i_{r}\right\} \subseteq\{1,2, \ldots, n\}
$$

with $i_{1}<i_{2}, \ldots, i_{r}$ we define

$$
\lambda(I)=\left(i_{r}-r+1, i_{r-1}-r+2, \ldots, i_{2}-1, i_{1}\right) .
$$

Theorem 1.1. The set $\mathcal{K}_{n} \subseteq \mathbb{R}^{3 n}$ is given by the trace equation (1), the weakly decreasing conditions (2) and all inequalities $\left(\star_{I, J, K}\right)$ (see (3)) with $0<r:=|I|=|J|=|K|<n$, such that

$$
(\lambda(I), \lambda(J), \lambda(K)) \in \mathcal{K}_{r}
$$

The theorem reflects the recursive nature of the cones $\mathcal{K}_{n}$. Once we have determined the cones $\mathcal{K}_{1}, \ldots, \mathcal{K}_{n-1}$, we can determine a system of inequalities for the cone $\mathcal{K}_{n}$.

A crucial part of the solution of Horn's conjecture is its connection to the representation theory of $\mathrm{GL}_{n}(\mathbb{C})$. Irreducible representations $V_{\lambda}$ of $\mathrm{GL}_{n}(\mathbb{C})$ are parameterized by non-increasing integer sequences $\lambda=\left(\lambda_{1}, \ldots, \lambda_{n}\right)$. The Littlewood-Richardson coefficient $c_{\lambda, \mu}^{\nu}$ is defined as the multiplicity of $V_{\nu}$ inside the tensor product $V_{\lambda} \otimes V_{\mu}$, i.e.,

$$
c_{\lambda, \mu}^{\nu}:=\operatorname{dim}\left(V_{\lambda} \otimes V_{\mu} \otimes V_{\nu}^{\star}\right)^{\mathrm{GL}_{n}(\mathbb{C})} .
$$

Here $V_{\nu}^{\star}$ denotes the dual space of $V_{\nu}$ and $\left(V_{\lambda} \otimes V_{\mu} \otimes V_{\nu}^{\star}\right)^{\mathrm{GL}_{n}(\mathbb{C})}$ denotes the $\mathrm{GL}_{n}(\mathbb{C})$-invariant tensors in $V_{\lambda} \otimes V_{\mu} \otimes V_{\nu}^{\star}$. We define $c_{\lambda, \mu}^{\nu}=0$ if $\lambda, \mu, \nu$ are not weakly decreasing. Let us define

$$
\mathcal{L} R_{n}=\left\{(\lambda, \mu, \nu) \in\left(\mathbb{Z}^{n}\right)^{3} \mid c_{\lambda, \mu}^{\nu} \neq 0\right\} .
$$

The following results follow from Klyachko's paper [27].

Theorem 1.2. Let $\mathbb{R}_{+}$be the set of nonnegative real numbers. The cone $\mathbb{R}_{+} \mathcal{L} R_{n} \subseteq \mathbb{R}^{3 n}$ is equal to $\mathcal{K}_{n}$.

Theorem 1.3. The set $\mathcal{K}_{n} \subseteq \mathbb{R}^{3 n}$ is given by the trace equation (1), the weakly decreasing conditions (2) and all inequalities $\left(\star_{I, J, K}\right)$ with $0<r:=|I|=|J|=|K|<n$, such that

$$
(\lambda(I), \lambda(J), \lambda(K)) \in \mathcal{L} R_{r}
$$

Finally, the missing link for Theorem 1.1 is proved by Knutson and Tao in [28].

Theorem 1.4 (Saturation Theorem). The set $\mathcal{L} R_{n} \subseteq \mathbb{Z}^{3 n}$ is saturated, i.e.,

$$
\mathcal{L} R_{n}=\mathbb{R}_{+} \mathcal{L} R_{n} \cap \mathbb{Z}^{3 n} .
$$


The Saturation Theorem can also be formulated as follows: if $\lambda, \mu, \nu \in \mathbb{Z}^{n}$ such that $c_{N \lambda, N \mu}^{N \nu} \neq 0$ for some positive integer $N$, then $c_{\lambda, \mu}^{\nu} \neq 0$. By Theorems 1.2 and 1.4 we have $\mathcal{L} R_{n}=\mathcal{K}_{n} \cap \mathbb{Z}^{3 n}$. Combining this with Theorem 1.3 implies Theorem[1.1. In [28, Knutson and Tao use their Honeycomb model to prove Theorem [1.4. See also [5] for another version of the proof. A geometric proof of Theorem 1.4 was given by the authors in [13] and by Belkale in [3].

By Theorem 1.3 the set $\mathcal{K}_{n}$ is defined by (11), (2) and all inequalities $\left(\star_{I, J, K}\right)$ for which $c_{\lambda(I), \lambda(J)}^{\lambda(K)} \neq 0$ are nonzero. C. Woodward was first to note that some of these inequalities are redundant: they follow from the other inequalities. P. Belkale proved that all inequalities $\left(\star_{I, J, K}\right)$ for which $c_{\lambda(I), \lambda(J)}^{\lambda(K)}>1$ are redundant. This class includes the examples found by Woodward. As the following theorem by Knutson, Tao and Woodward ([29]) shows, none of the remaining inequalities can be omitted.

Theorem 1.5. For $n \geq 3, \mathcal{K}_{n}$ is defined by the equation (11), the inequalities (2) and all inequalities $\left(\star_{I, J, K}\right)$ for which $c_{\lambda(I), \lambda(J)}^{\lambda(K)}=1$. None of the inequalities the inequalities can be omitted.

For $n=2$, some of the inequalities (2) can be omitted. The cone $\mathcal{K}_{n}$ has dimension $3 n-1$. The inequality

$$
\left(\star_{I, J, K}\right): \sum_{i \in I} \lambda_{i}+\sum_{j \in J} \mu_{j} \geq \sum_{k \in K} \nu_{k}
$$

is necessary if and only if the hyperplane section

$$
\left\{(\lambda, \mu, \nu) \in\left(\mathbb{R}^{n}\right)^{3} \mid \sum_{i \in I} \lambda_{i}+\sum_{j \in J} \mu_{j}=\sum_{k \in K} \nu_{k}\right\} \cap \mathcal{K}_{n}
$$

defines a facet (or wall) of the cone $\mathcal{K}_{n}$.

Along the way, Knutson, Tao and Woodward also proved (see 29]) the following Theorem, which was conjectured by W. Fulton.

Theorem 1.6. If $c_{\lambda, \mu}^{\nu}=1$ for some $\lambda, \mu, \nu$, then $c_{N \lambda, N \mu}^{N \nu}=1$ for all nonnegative integers $N$.

A geometric proof of Theorem [1.6 using Schubert calculus was given by P. Belkale in [4].

1.3. The quiver method. A quiver is just a directed graph. If we attach vector spaces to the vertices and linear maps to the arrows, we get a representation of that graph.

Let $Q$ be a quiver without oriented cycles and $K$ be an algebraically closed field. Suppose that $\alpha \in \mathbb{N}^{Q_{0}}$, where $\mathbb{N}=\{0,1,2, \ldots\}, Q_{0}$ is the set of vertices of the quiver and $\mathbb{N}^{Q_{0}}$ is the set of dimension vectors. In [13] the authors studied the set $\Sigma(Q, \alpha) \subseteq \mathbb{Z}^{Q_{0}}$ of weights occurring in the ring of semi-invariants on the space of $\alpha$-dimensional representations $\operatorname{Rep}(Q, \alpha)$ over the field $K$. We showed that this set is given by one linear homogeneous equation and a finite set of homogeneous linear inequalities. Thus the positive real span $\mathbb{R}_{+} \Sigma(Q, \alpha) \subseteq \mathbb{R}^{Q_{0}}$ forms a rational polyhedral cone in $\mathbb{R}^{Q_{0}}$. In the particular case of the triple flag quiver $T_{n, n, n}$ and a special dimension vector $\beta$ the cone $\mathbb{R}_{+} \Sigma\left(T_{n, n, n}, \beta\right)$ turns out to be equal to the cone $\mathbb{R}_{+} \mathcal{L} R_{n}$ defined above. Thus it is interesting to study the properties of the general cones $\mathbb{R}_{+} \Sigma(Q, \alpha)$, in particular whether the statements listed above concerning the cones $\mathbb{R}_{+} \mathcal{L} R_{n}$ generalize to arbitrary quivers. If this is true, one could expect that such technique would allow to prove stronger results about the cones $\mathbb{R}_{+} \mathcal{L} R_{n}$.

This program is carried out in the present paper for arbitrary quiver $Q$ without oriented cycles. The approach is based on studying the notions of semi-stable filtrations from Geometric Invariant Theory in terms of quiver representations. As a result of this study we generalize Theorems 1.4. 1.5 and 1.6 above to arbitrary cones $\mathbb{R}_{+} \Sigma(Q, \alpha)$. We also obtain combinatorial description of faces of all codimensions in the cones $\mathbb{R}_{+} \Sigma(Q, \alpha)$. For the case of faces of codimension one this is equivalent to Theorem 1.5. In the special case of extremal rays the results imply that the semi-invariants 
with weights lying on an extremal ray of $\mathbb{R}_{+} \Sigma(Q, \alpha)$ form a subring isomorphic to the ring of semiinvariants for quivers with two vertices and multiple arrows. This indicates that one needs to study all quivers, not just the special class of triple flag quivers, so the quiver technique is the right tool for studying similar questions. The cones $\mathbb{R}_{+} \mathcal{L} R_{n}$ are also related to a problem of existence of short exact sequences of abelian $p$-groups (see [19, 26]). Recent results of C. Chindris ([6, 7]) show that quivers can be successfully applied to get similar results for longer exact sequences.

We use the developed techniques to prove some new results on the faces of cones $\mathbb{R}_{+} \mathcal{L} R_{n}$, in particular a product formula for Littlewood-Richardson coefficients. The general approach explains why one could expect such formula.

1.4. Organization. The paper is organized as follows. In Section 2 we give the basic notation and review the needed results on quivers and their semi-invariants. In particular we review the content of papers [9], [13, 38] and [39], in particular the Saturation Theorem for the cones $\mathbb{R}_{+} \Sigma(Q, \alpha)$, the exceptional sequences and orthogonal categories.

There are some reformulations and extensions, notably the Embedding Theorem 2.39, Schofield's technique of orthogonal categories allows to embed the category of representations of a smaller quiver (not necessarily a subquiver) into the category of representations of the original quiver. Theorem 2.39 shows that this embedding respects semi-invariants. This gives us a method for proving results about the cones $\mathbb{R}_{+} \Sigma(Q, \alpha)$ by induction. This technique requires one to work with arbitrary quivers, not just triple flag quivers.

We also formulate the Generalized Fulton's Conjecture (Theorem 2.23) whose proof (essentially due to P. Belkale, see [4) is given in the Appendix.

In Section 3 we study the notions of semi-stability and stability of quiver representations. We relate the geometric notion of $(\sigma: \tau)$-stability and the algebraic notion of $\sigma$-stability, using the approach of 34. We introduce the notions of Harder-Narasimhan, Jordan-Hölder filtrations, and their combination - the HNJH filtrations. The key statement is Lemma 3.8 which shows that the subsets of the representation space $\operatorname{Rep}(Q, \alpha)$ where the dimensions of the factors of Harder-Narasimhan and HNJH filtrations are constant are locally closed. Then, filtrations are used to define the $\sigma$-stable decompositions and $(\sigma: \tau)$-stable decompositions of dimension vectors, and to prove their basic properties. In particular, Theorem 3.20 relates the semi-invariants in weights $m \sigma$ for a dimension vector $\alpha$ to those for the dimension vectors that are factors in $\sigma$-stable decomposition of $\alpha$.

In Section 4 we introduce the key notions of Schur sequences and Schur quiver sequences, inspired by the notion of exceptional sequences. These notions provide the tools for the descriptions of the faces of the cones $\mathbb{R}_{+} \Sigma(Q, \alpha)$. Then we prove the Refinement Theorem (Theorem 4.11) which says that every Schur sequence can be refined to an exceptional sequence. This theorem makes it easy to understand Schur sequences in terms of exceptional sequences.

In Section 5 we prove the basic Theorem 5.1 giving a bijection between the faces of dimension $n-r$ in $\Sigma(Q, \alpha)$, and Schur quiver sequences of $r$ dimension vectors summing to $\alpha$. This is the main result of the paper. Then we draw consequences for faces of codimension 1 and for extremal rays.

In Section 6 we study the dual problem of how the $\sigma$-stable decomposition of $\alpha$ varies when $\alpha$ varies and $\sigma$ is fixed. Theorem 6.4 gives a general combinatorial criterion of when $\alpha$ is $\sigma$-stable. We also extend the notion of $\sigma$-stable decomposition to quivers with oriented cycles.

In Section 7 we apply our results to triple flag quivers and the cones $\mathbb{R}_{+} \mathcal{L} R_{n}$. We recover Theorem 1.5 of Knutson, Tao and Woodward on faces of codimension one.

We also investigate the faces of $\mathbb{R}_{+} \mathcal{L} R_{n}$ of arbitrary codimension. In particular we show that for $n \leq 7$ all weight multiplicities along extremal rays of the cones $\mathbb{R}_{+} \mathcal{L} R_{n}$ are equal to 1 , and for $n=8$ we give an example of an extremal ray with weight multiplicities bigger than 1 .

Finally we prove the product formula for Littlewood-Richardson coefficients (Theorem 7.14 ). It shows that if a weight $\sigma$ corresponding to a triple of partitions $(\lambda, \mu, \nu)$ lies on a face of $\Sigma\left(T_{n, n, n}, \beta\right)$ 
of positive codimension, then the Littlewood-Richardson coefficient $c_{\lambda, \mu}^{\nu}$ decomposes to a product of smaller Littlewood-Richardson coefficients.

Acknowledgment. Both authors would like to thank Prakash Belkale for the permission to include his proof of the Fulton Conjecture, Calin Chindris for helpful discussions, and Jiarui Fei for proofreading.

\section{Preliminaries}

2.1. Basic notions for quivers. A quiver $Q$ is a quadruple $Q=\left(Q_{0}, Q_{1}, h, t\right)$ where $Q_{0}$ is a finite set of vertices, $Q_{1}$ is a finite set of arrows and $h, t: Q_{1} \rightarrow Q_{0}$ are maps. For each arrow $a \in Q_{1}$, its head is $h a:=h(a) \in Q_{0}$ and its tail is $t a:=t(a) \in Q_{1}$.

We fix an algebraically closed field $K$. A representation $V$ of $Q$ is a family of finite dimensional $K$-vector spaces

$$
\left\{V(x) \mid x \in Q_{0}\right\}
$$

together with a family of $K$-linear maps

$$
\left\{V(a): V(t a) \rightarrow V(h a) \mid a \in Q_{1}\right\} .
$$

The dimension vector of a representation $V$ is the function $\underline{\operatorname{dim}} V: Q_{0} \rightarrow \mathbb{N}$ defined by

$$
(\underline{\operatorname{dim}} V)(x):=\operatorname{dim} V(x), \quad x \in Q_{0} .
$$

The dimension vectors $\mathbb{N}^{Q_{0}}$ are contained in the set $\Gamma:=\mathbb{Z}^{Q_{0}}$ of integer-valued functions on $Q_{0}$. A morphism $\phi: V \rightarrow W$ between two representations is the collection of linear maps

$$
\left\{\phi(x): V(x) \rightarrow W(x) \mid x \in Q_{0}\right\}
$$

such that for each $a \in Q_{1}$ we have

$$
W(a) \phi(t a)=\phi(h a) V(a) .
$$

We denote the (finite dimensional) linear space of morphisms from $V$ to $W$ by $\operatorname{Hom}_{Q}(V, W)$.

A nontrivial path $p$ in the quiver $Q$ of length $n \geq 1$ is a sequence $p=a_{n} a_{n-1} \cdots a_{1}$ of arrows, such that $t a_{i+1}=h a_{i}$ for $i=1,2, \ldots, n-1$. We define the head and the tail of the path $p$ as $h p:=h a_{n}$ and $t p:=t a_{1}$ respectively. Besides the nontrivial paths one usually also defines a trivial path $\epsilon_{x}$ of length 0 for every vertex $x \in Q_{0}$ whose head and tail are equal to $h \epsilon_{x}=t \epsilon_{x}=x$. An oriented cycle is a nontrivial path $p$ satisfying $h p=t p$. Throughout this paper, we will assume that $Q$ has no oriented cycles, unless stated otherwise.

The category $\operatorname{Rep}(Q)=\operatorname{Rep}_{K}(Q)$ of representations of $Q$ over $K$ is hereditary, i.e., the subobject of a projective object is projective. This means that every representation has projective dimension $\leq 1$, i.e., $\operatorname{Ext}_{Q}^{i}(V, W)=0$ for all representations $V, W$ and all $i>1$.

Lemma 2.1 (See [35]). The spaces $\operatorname{Hom}_{Q}(V, W)$ and $\operatorname{Ext}_{Q}(V, W):=\operatorname{Ext}_{Q}^{1}(V, W)$ are the kernel and cokernel of the following linear map

$$
d_{W}^{V}: \bigoplus_{x \in Q_{0}} \operatorname{Hom}(V(x), W(x)) \longrightarrow \bigoplus_{a \in Q_{1}} \operatorname{Hom}(V(t a), W(h a))
$$

where $d_{W}^{V}$ is given by

$$
\left\{\phi(x) \mid x \in Q_{0}\right\} \mapsto\left\{W(a) \phi(t a)-\phi(h a) V(a) \mid a \in Q_{1}\right\} .
$$

Let $\alpha, \beta$ be two elements of $\Gamma$. We define the Euler inner product (or Ringel form) by

$$
\langle\alpha, \beta\rangle_{Q}=\sum_{x \in Q_{0}} \alpha(x) \beta(x)-\sum_{a \in Q_{1}} \alpha(t a) \beta(h a) .
$$


It follows from Lemma 2.1 and (5) that

$$
\langle\underline{\operatorname{dim}} V, \underline{\operatorname{dim}} W\rangle_{Q}=\operatorname{dim} \operatorname{Hom}_{Q}(V, W)-\operatorname{dim} \operatorname{Ext}_{Q}(V, W) .
$$

We will omit the subscript $Q$ and just write $\langle\cdot, \cdot\rangle$ instead of $\langle\cdot, \cdot\rangle_{Q}$ if there is no chance of confusion.

2.2. Semi-invariants for quiver representations. Suppose that $V$ is a $\beta$-dimensional representation. Choose a basis of each of the vector spaces $V(x), x \in Q_{0}$. The matrix of $V(a)$ with respect to the bases in $V(t a)$ and $V(h a)$ is an element of $\operatorname{Mat}_{\beta(h a), \beta(t a)}(K)$, where $\operatorname{Mat}_{p, q}(K)$ denotes the $p \times q$ matrices with entries in $K$. This way we can associate to $V$ an element of the representation space

$$
\operatorname{Rep}(Q, \beta):=\prod_{a \in Q_{1}} \operatorname{Mat}_{\beta(h a), \beta(t a)}(K)
$$

The group

$$
\operatorname{GL}(Q, \beta):=\prod_{x \in Q_{0}} \operatorname{GL}_{\beta(x)}(K)
$$

acts on $\operatorname{Rep}(Q, \beta)$ as follows

$$
\left\{A(x) \mid x \in Q_{0}\right\} \cdot\left\{V(a) \mid a \in Q_{1}\right\}:=\left\{A(h a) V(a) A(t a)^{-1} \mid a \in Q_{1}\right\},
$$

for $\left\{A(x) \mid x \in Q_{0}\right\} \in \operatorname{GL}(Q, \beta)$ and $\left\{V(a) \mid a \in Q_{1}\right\} \in \operatorname{Rep}(Q, \beta)$. The action of $\operatorname{GL}(Q, \beta)$ on $\operatorname{Rep}(Q, \beta)$ corresponds to base changes in each of the vector spaces $V(x) \cong K^{\beta(x)}, x \in Q_{0}$. The orbits of $\operatorname{GL}(Q, \beta)$ in $\operatorname{Rep}(Q, \beta)$ correspond to the isomorphism classes of $\beta$-dimensional representations of $Q$.

Define $\operatorname{SL}(Q, \beta) \subseteq \mathrm{GL}(Q, \beta)$ by

$$
\operatorname{SL}(Q, \beta)=\prod_{x \in Q_{0}} \operatorname{SL}_{\beta(x)}(K) .
$$

We are interested in the rings of semi-invariants

$$
\operatorname{SI}(Q, \beta)=K[\operatorname{Rep}(Q, \beta)]^{\operatorname{SL}(Q, \beta)} .
$$

The ring $\operatorname{SI}(Q, \beta)$ has a weight space decomposition

$$
\operatorname{SI}(Q, \beta)=\bigoplus_{\sigma} \operatorname{SI}(Q, \beta)_{\sigma}
$$

where $\sigma$ runs through the multiplicative characters of $\operatorname{GL}(Q, \beta)$ and

$$
\operatorname{SI}(Q, \beta)_{\sigma}=\{f \in K[\operatorname{Rep}(Q, \beta)] \mid g(f)=\sigma(g) f \forall g \in \operatorname{GL}(Q, \beta)\} .
$$

Any character or weight of $\mathrm{GL}(Q, \beta)$ has the form

$$
\left\{A(x) \mid x \in Q_{0}\right\} \mapsto \prod_{x \in Q_{0}}(\operatorname{det} A(x))^{\sigma(x)}
$$

with $\sigma(x) \in \mathbb{Z}$ for all $x \in Q_{0}$. This way, the multiplicative character (7) of $\operatorname{GL}(Q, \beta)$ can be identified with $\sigma \in \Gamma:=\mathbb{Z}^{Q_{0}}$.

If $\alpha \in \Gamma$ then we define

$$
\sigma(\alpha)=\sum_{x \in Q_{0}} \sigma(x) \alpha(x) .
$$

We will identify the set of weights with $\Gamma^{\star}=\operatorname{Hom}_{\mathbb{Z}}(\Gamma, \mathbb{Z}) \cong \mathbb{Z}^{Q_{0}}$. Note that $\Gamma^{\star}$ and $\Gamma$ are canonically isomorphic, but we still would like to distinguish between them.

Let us choose the dimension vectors $\alpha, \beta \in \mathbb{N}^{Q_{0}}$ such that $\langle\alpha, \beta\rangle=0$. If $V \in \operatorname{Rep}(Q, \alpha)$ and $W \in \operatorname{Rep}(Q, \beta)$, then the matrix of $d_{W}^{V}$ in (4) is a square matrix. Following [38] we define the semi-invariant

$$
c(V, W):=\operatorname{det} d_{W}^{V}
$$


of the action of $\operatorname{GL}(Q, \alpha) \times \operatorname{GL}(Q, \beta)$ on $\operatorname{Rep}(Q, \alpha) \times \operatorname{Rep}(Q, \beta)$. For a fixed $V$ the restriction of $c$ to $\{V\} \times \operatorname{Rep}(Q, \beta)$ defines a semi-invariant $c^{V}$ in $\operatorname{SI}(Q, \beta)$. Schofield proved ([38, Lemma 1.4]) that the weight of $c^{V}$ equals $\langle\alpha, \cdot\rangle$. Note that $\langle\alpha, \cdot\rangle$ can be viewed as an element in $\Gamma^{\star}$. Similarly, for a fixed $W$ the restriction of $c$ to $\operatorname{Rep}(Q, \alpha) \times\{W\}$ defines a semi-invariant $c_{W}$ in $\operatorname{SI}(Q, \alpha)$ of weight $-\langle\cdot, \beta\rangle$ ([38, Lemma 1.4]).

Lemma 2.2 (Lemma 1 of [13]). Suppose that

$$
0 \rightarrow V_{1} \rightarrow V \rightarrow V_{2} \rightarrow 0
$$

is an exact sequence of representations of $Q$ and $\left\langle\underline{\operatorname{dim}} V_{1}, \beta\right\rangle=\left\langle\underline{\operatorname{dim}} V_{2}, \beta\right\rangle=0$, then as a function on $\operatorname{Rep}(Q, \beta), c^{V}$ is, up to a scalar, equal to $c^{V_{1}} \cdot c^{V_{2}}$.

Theorem 2.3 (Theorem 1 of $[13)$. The ring $\operatorname{SI}(Q, \beta)$ is spanned by semi-invariants of the form $c^{V}$ for which $\langle\underline{\operatorname{dim}} V, \beta\rangle=0$. It is also spanned by semi-invariants of the form $c_{W}$ for which $\langle\beta, \underline{\operatorname{dim}} W\rangle=0$.

For a more general statement for quivers with oriented cycles, see [15, 41].

Remark 2.4. If $\langle\underline{\operatorname{dim}} V, \underline{\operatorname{dim}} W\rangle=0$ then we have $c(V, W)=c^{V}(W)=c_{W}(V)=0$ if and only if $\operatorname{Hom}_{Q}(V, W) \neq 0$ which is equivalent to $\operatorname{Ext}_{Q}(V, W) \neq 0$ by Lemma 2.1.

It was also shown in [13] that

$$
\operatorname{dim} \operatorname{SI}(Q, \beta)_{\langle\alpha, \cdot\rangle}=\operatorname{dim} \operatorname{SI}(Q, \alpha)_{-\langle\cdot, \beta\rangle} .
$$

Definition 2.5. For dimension vectors $\alpha, \beta$ with $\langle\alpha, \beta\rangle=0$ we define

$$
(\alpha \circ \beta)_{Q}:=\operatorname{dim} \operatorname{SI}(Q, \beta)_{\langle\alpha, \cdot\rangle}=\operatorname{dim} \operatorname{SI}(Q, \alpha)_{-\langle\cdot, \beta\rangle} .
$$

Again, we will drop the subscript $Q$ most of the time and write $\alpha \circ \beta$ instead of $(\alpha \circ \beta)_{Q}$.

2.3. Representations in general position. A representation $V$ is called indecomposable if it is not isomorphic to the direct sum of two nonzero representations. The set of dimension vectors $\alpha$ for which there exists an $\alpha$-dimensional indecomposable representation can be identified with the set of positive roots for the Kac-Moody algebra associated with the graph $Q$ (where we forget the orientation). This was proved in 22. We will call a dimension vector $\alpha$ a root if there exists an indecomposable representation of dimension $\alpha$. Kac proved that $\langle\alpha, \alpha\rangle \leq 1$ for every root $\alpha$. If $\alpha$ is a root, then we will call $\alpha$ real if $\langle\alpha, \alpha\rangle=1$ and imaginary if $\langle\alpha, \alpha\rangle \leq 0$. We call $\alpha$ isotropic if $\langle\alpha, \alpha\rangle=0$.

A representation $V$ is called a Schur representation (or a brick) if $\operatorname{Hom}_{Q}(V, V) \cong K$. Note that every Schur representation must be indecomposable. If $\operatorname{Rep}(Q, \alpha)$ contains a Schur representation, then $\alpha$ is called a Schur root.

A representation $V$ is called in general position of dimension $\alpha$ if $V \in \operatorname{Rep}(Q, \alpha)$ lies in a sufficiently small Zariski open subset ("sufficient" here depends on the context). Suppose that $\alpha$ is a Schur root. Since $V \mapsto \operatorname{dim} \operatorname{Hom}_{Q}(V, V)$ depends upper semi-continuously on $V \in \operatorname{Rep}(Q, \alpha)$, its minimal value 1 is attained on some open dense subset $U \subseteq \operatorname{Rep}(Q, \alpha)$. This shows that a general representation of $\operatorname{Rep}(Q, \alpha)$ is indecomposable. Conversely, if a general representation of dimension $\alpha$ is indecomposable, then $\alpha$ must be a Schur root (see [23, Proposition 1]).

We define

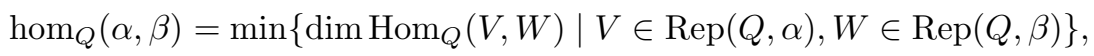

where min denotes the minimum. The function $(V, W) \mapsto \operatorname{dim}_{\operatorname{Hom}}(V, W)$ is upper semi-continuous, so $\operatorname{dim} \operatorname{Hom}_{Q}(V, W)=\operatorname{hom}_{Q}(\alpha, \beta)$ if $(V, W) \in \operatorname{Rep}(Q, \alpha) \times \operatorname{Rep}(Q, \beta)$ is in general position (see [39]). Similarly, we define

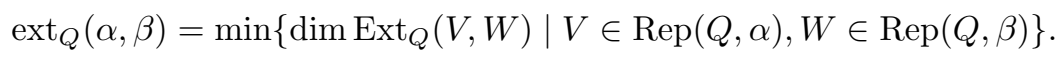


We will drop the subscript and write $\operatorname{hom}(\alpha, \beta)$ and $\operatorname{ext}(\alpha, \beta)$ if there is no confusion. From (5) follows that

$$
\langle\alpha, \beta\rangle=\operatorname{hom}(\alpha, \beta)-\operatorname{ext}(\alpha, \beta) .
$$

Definition 2.6. If $\operatorname{hom}_{Q}(\alpha, \beta)=\operatorname{ext}_{Q}(\alpha, \beta)=0$, then we write $\alpha \perp \beta$ and we will say that $\alpha$ is left perpendicular to $\beta$.

By Remark 2.4 we have $\alpha \perp \beta$ if and only if $\alpha \circ \beta \neq 0$. Following Schofield, we write $\alpha \hookrightarrow \beta$ if a general representation of dimension $\beta$ contains a subrepresentation of dimension $\alpha$. We write $\alpha \rightarrow \beta$ if a general representation of dimension $\alpha$ has a factor of dimension $\beta$. The proof of the following Theorem can be found in [39] for a base field of characteristic 0 . For a proof that also works in positive characteristic, see [10].

Theorem 2.7 (Theorem 3.3 of [39]). We have

$$
\alpha \hookrightarrow \alpha+\beta \quad \Leftrightarrow \quad \operatorname{ext}_{Q}(\alpha, \beta)=0 \quad(\Leftrightarrow \alpha+\beta \rightarrow \beta) .
$$

Definition 2.8. For a dimension vector $\beta$, we define

$$
\Sigma(Q, \beta)=\left\{\sigma \in \Gamma^{\star} \mid \mathrm{SI}(Q, \beta)_{\sigma} \neq 0\right\} .
$$

Theorem 2.9 (see [13]). We have

$$
\Sigma(Q, \beta)=\left\{\sigma \in \Gamma^{\star} \mid \sigma(\beta)=0 \text { and } \sigma(\gamma) \leq 0 \text { for all } \gamma \hookrightarrow \beta\right\} .
$$

For some $\gamma \hookrightarrow \beta$, the inequality $\sigma(\gamma) \leq 0$ can be omitted because it follows from the other inequalities. Later, we will describe a minimal list of inequalities for $\Sigma(Q, \beta)$.

Theorem 2.10 (see [16]). Suppose that $\alpha, \beta$ are dimension vectors satisfying $\operatorname{hom}(\alpha, \beta)=\operatorname{ext}(\alpha, \beta)=$ 0 . Then a general representation of dimension $\alpha+\beta$ has exactly $\alpha \circ \beta$ subrepresentations of dimension $\alpha$.

Lemma 2.11. Under the assumptions of Theorem [2.9, if $V \in \operatorname{Rep}(Q, \alpha+\beta)$ is arbitrary such that $V$ has exactly $r$ subrepresentations, where $r$ is finite, then $r \leq \alpha \circ \beta$.

Proof. Schofield constructs a variety $Z:=R(Q, \alpha \subset \alpha+\beta)$ (see [39]) and a projective morphism $p: Z \rightarrow \operatorname{Rep}(Q, \alpha+\beta)$ such that the fiber $p^{-1}(V)$ of $V \in \operatorname{Rep}(Q, \alpha+\beta)$ can be identified with the set of all subrepresentations of $V$. Let $U \subseteq \operatorname{Rep}(Q, \alpha+\beta)$ be the set of all $V \in \operatorname{Rep}(Q, \alpha+\beta)$ such that the fiber $p^{-1}(V)$ is finite. Because $p$ is projective it follows by the semi-continuity of the dimension of a fiber that $U$ is open. Let us restrict $p$ to $p^{-1}(U) \rightarrow U$. Now $p: p^{-1}(U) \rightarrow U$ is a projective, quasi-finite map, hence it is finite. It follows that all fibers have the same cardinality if counted with multiplicity. It was shown in [10 that a general fiber of $p$ is reduced (this is not immediately clear in positive characteristic). Therefore, a general fiber is, set-theoretically, the largest among all fibers $p^{-1}(V), V \in U$.

2.4. The canonical decomposition. Following Kac, we make the following definition.

Definition 2.12 (Section 4 of [23]). We call

$$
\alpha=\alpha_{1} \oplus \alpha_{2} \oplus \cdots \oplus \alpha_{s}
$$

the canonical decomposition of $\alpha$ if a general representation of dimension $\alpha$ decomposes into indecomposable representations of dimensions $\alpha_{1}, \alpha_{2}, \ldots, \alpha_{s}$.

For more details on the canonical decomposition, see [23, 14. 
Theorem 2.13 (Proposition 3 of [23]). The expression

$$
\alpha=\alpha_{1} \oplus \alpha_{2} \oplus \cdots \oplus \alpha_{s}
$$

is the canonical decomposition if and only if $\alpha_{1}, \ldots, \alpha_{s}$ are Schur roots, and $\operatorname{ext}\left(\alpha_{i}, \alpha_{j}\right)=0$ for all $i \neq j$.

Theorem 2.14 (Theorem 3.8 of [39]). Suppose that $\alpha$ has the canonical decomposition

$$
\alpha_{1} \oplus \alpha_{2} \oplus \cdots \oplus \alpha_{s}
$$

and $p \in \mathbb{N}$. Then the canonical decomposition of $p \alpha$ is

$$
\left[p \alpha_{1}\right] \oplus\left[p \alpha_{2}\right] \oplus \cdots \oplus\left[p \alpha_{s}\right]
$$

where

$$
[p \beta]= \begin{cases}\beta^{\oplus p}:=\underbrace{\beta \oplus \cdots \oplus \beta}_{p} & \text { if } \beta \text { is a real or isotropic Schur root; } \\ p \beta & \text { if } \beta \text { is a non-isotropic imaginary Schur root. }\end{cases}
$$

Lemma 2.15 (Lemma 5.2 of [40]). Suppose that

$$
\alpha=\alpha_{1}^{\oplus r_{1}} \oplus \alpha_{2}^{\oplus r_{2}} \oplus \cdots \oplus \alpha_{s}^{\oplus r_{s}}
$$

is the canonical decomposition of $\alpha$, where $\alpha_{1}, \alpha_{2}, \ldots, \alpha_{s}$ are distinct dimension vectors and $r_{1}, \ldots, r_{s}$ are positive integers. Then we may assume, after rearranging $\alpha_{1}, \ldots, \alpha_{s}$, that $\operatorname{hom}_{Q}\left(\alpha_{i}, \alpha_{j}\right)=0$ for all $i<j$.

In [14 an efficient algorithm was given to compute the canonical decomposition of a given dimension vector. A similar recursive procedure was given in in 40. Lemma 2.15 follows immediately from the correctness of the algorithm in [14, because the output of the algorithm has the desired property.

For a representation $V \in \operatorname{Rep}(Q, \alpha)$, we have

$$
\langle\alpha, \alpha\rangle=\sum_{x \in Q_{0}} \alpha(x)^{2}-\sum_{a \in Q_{1}} \alpha(t a) \alpha(h a)=\operatorname{dim} \operatorname{GL}(Q, \alpha)-\operatorname{dim} \operatorname{Rep}(Q, \alpha) .
$$

On the other hand,

$$
\operatorname{dim} \operatorname{GL}(Q, \alpha)=\operatorname{dim} \operatorname{GL}(Q, \alpha)_{V}+\operatorname{dim} \operatorname{GL}(Q, \alpha) \cdot V
$$

where $\operatorname{GL}(Q, \alpha)_{V}$ is the stabilizer of $V$ and $\operatorname{GL}(Q, \alpha) \cdot V$ is the orbit of $V$. Because $\operatorname{GL}(Q, \alpha)_{V}$ is equal to the invertible elements of $\operatorname{Hom}_{Q}(V, V)$, it follows from (6) that

$$
\operatorname{dim} \operatorname{GL}(Q, \alpha)_{V}=\operatorname{dim} \operatorname{Hom}_{Q}(V, V)=\langle\alpha, \alpha\rangle+\operatorname{dim} \operatorname{Ext}_{Q}(V, V) .
$$

Adding (9), (10) and (11) yields

$$
\operatorname{dim} \operatorname{Rep}(Q, \alpha)-\operatorname{dim} \operatorname{GL}(Q, \alpha) \cdot V=\operatorname{dim} \operatorname{Ext}(V, V)
$$

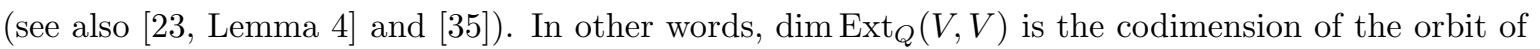
$V$ in $\operatorname{Rep}(Q, \alpha)$.

Definition 2.16. If $\operatorname{Rep}(Q, \alpha)$ contains a dense $\operatorname{GL}(Q, \alpha)$-orbit, then $\alpha$ is called a prehomogeneous dimension vector.

From (12) follows that $\alpha$ is prehomogeneous if and only if there exists a representation $V \in$ $\operatorname{Rep}(Q, \alpha)$ satisfying $\operatorname{Ext}_{Q}(V, V)=0$.

Let us prove the following known fact.

Lemma 2.17. If $\alpha$ is a real Schur root, then $\alpha$ is prehomogeneous.

Proof. Suppose that $\alpha$ is a real Schur root and $V \in \operatorname{Rep}(Q, \alpha)$ is a Schur representation. From

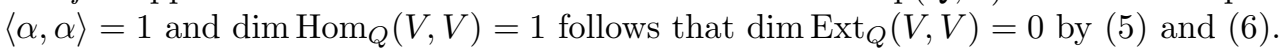


Theorem 2.18 (See Proposition 4 in [23]). Suppose that

$$
\alpha=\alpha_{1} \oplus \alpha_{2} \oplus \cdots \oplus \alpha_{s}
$$

is the canonical decomposition of $\alpha$. Then $\alpha$ is prehomogeneous if and only of $\alpha_{1}, \ldots, \alpha_{s}$ are real Schur roots.

2.5. The combinatorics of dimension vectors. We point out that many of the notions we just introduced can be defined combinatorially. For example the quantity $\operatorname{ext}(\alpha, \beta)$ can be, in principle, computed using a recursive procedure using the following result.

Theorem 2.19 (Theorem 5.4 of [39]). We have

$$
\begin{gathered}
\operatorname{ext}(\alpha, \beta)=\max \left\{-\left\langle\alpha^{\prime}, \beta^{\prime}\right\rangle \mid \alpha^{\prime} \hookrightarrow \alpha, \beta \rightarrow \beta^{\prime}\right\}= \\
=\max \left\{-\left\langle\alpha, \beta^{\prime}\right\rangle \mid \beta \rightarrow \beta^{\prime}\right\}=\max \left\{-\left\langle\alpha^{\prime}, \beta\right\rangle \mid \alpha^{\prime} \hookrightarrow \alpha\right\} .
\end{gathered}
$$

By Theorem 2.7 the conditions $\alpha^{\prime} \hookrightarrow \alpha$ and $\beta \rightarrow \beta^{\prime}$ can be verified by computing ext-numbers for smaller dimension vectors.

Using (8) we can also compute $\operatorname{hom}(\alpha, \beta)$ recursively.

Corollary 2.20. The numbers $\operatorname{hom}(\alpha, \beta), \operatorname{ext}(\alpha, \beta)$ do not depend on the base field $K$.

Proposition 2.21. (see [16]) The numbers $\alpha \circ \beta$ do not depend on the base field $K$.

The numbers $\alpha \circ \beta$ can be computed either in terms of Schur functors, or equivalently, in terms of Schubert calculus. This way, $\alpha \circ \beta$ can be expressed as a (perhaps large) sum of products of Littlewood-Richardson coefficients. See [16] for more details.

The following definition will be important later.

Definition 2.22. Suppose that $\alpha, \beta \in \mathbb{N}^{Q_{0}}$. We say that two dimension vectors $\alpha, \beta$ are strongly perpendicular if

$$
\alpha \circ \beta=1
$$

We will denote this by $\alpha \Perp \beta$.

We have

$$
\alpha \Perp \beta \Rightarrow \alpha \perp \beta \Rightarrow\langle\alpha, \beta\rangle=0,
$$

and none of the implications can be reversed. The following result will be crucial for this paper.

Theorem 2.23 (Generalized Fulton Conjecture, Belkale, see the Appendix). If $\alpha \circ \beta=1$, then

$$
p \alpha \circ q \beta=1
$$

for all $p, q \in \mathbb{N}$.

Remark 2.24. Theorem 2.23 can be thought of as a generalization of Fulton's Conjecture (Theorem [1.6). For partitions $\lambda, \mu, \nu$ one can construct a quiver $Q$ and dimension vectors $\alpha, \beta$ such that $\alpha \circ \beta=c_{\lambda, \mu}^{\nu}$, and $\alpha \circ(n \beta)=c_{n \lambda, n \mu}^{n \nu}$. We will explain this in more detail in Section 7 .

A dimension vector $\alpha$ is a Schur root if and only if there exist no nonzero dimension vectors $\beta, \gamma$ with $\alpha=\beta+\gamma$ and $\operatorname{ext}(\beta, \gamma)=\operatorname{ext}(\gamma, \beta)=0$. Therefore, the set of Schur roots does not depend on the base field. 


\subsection{Perpendicular categories.}

Definition 2.25. A representation $V$ is called exceptional if $\operatorname{Hom}_{Q}(V, V) \cong K$ and $\operatorname{Ext}_{Q}(V, V)=0$.

If $V \in \operatorname{Rep}(Q, \alpha)$ is exceptional, then $V$ is a Schur representation and $\alpha$ is a Schur root. Moreover,

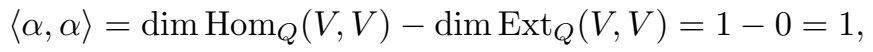

so $\alpha$ is a real Schur root. Conversely, if $\alpha$ is a real Schur root, then there exists a Schur representation $V \in \operatorname{Rep}(Q, \alpha)$. From

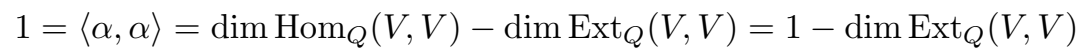

follows that $\operatorname{Ext}_{Q}(V, V)=0$. This means that the orbit $\operatorname{GL}(Q, \alpha) \cdot V$ is open and dense in $\operatorname{Rep}(Q, \alpha)$. Therefore, a general representation of dimension $\alpha$ is isomorphic to $V$. This shows that there is a natural bijection between real Schur roots and exceptional representations.

Definition 2.26. Suppose that $V$ is an exceptional representation. The right perpendicular category $V^{\perp}$ is the full subcategory of $\operatorname{Rep}_{K}(Q)$ whose objects are all representations $W$ such that $\operatorname{Hom}_{Q}(V, W)=\operatorname{Ext}_{Q}(V, W)=0$. Similarly, we define the left perpendicular category ${ }^{\perp} V$. as the full subcategory of $\operatorname{Rep}_{K}(Q)$ whose objects are all representations $W$ for which $\operatorname{Hom}_{Q}(W, V)=$ $\operatorname{Ext}_{Q}(W, V)=0$.

Note that if $V \perp W$ then $\underline{\operatorname{dim}} V \perp \underline{\operatorname{dim}} W$ (see Definition 2.6). Conversely, if $\alpha, \beta$ are dimension vectors with $\alpha \perp \beta$ then there exist $V \in \operatorname{Rep}(Q, \alpha)$ and $W \in \operatorname{Rep}(Q, \beta)$ with $V \perp W$.

The subcategory $V^{\perp}$ (respectively ${ }^{\perp} V$ ) are closed under taking kernels, cokernels, direct sums, images and extensions.

Theorem 2.27 (Theorem 2.2 of [38]). Suppose that $V$ is a sincere representation, i.e., $V(x) \neq 0$ for all $x \in Q_{0}$. Then the categories $V^{\perp}$ and ${ }^{\perp} V$ are equivalent.

The equivalence in the Theorem is given by the Auslander-Reiten transform. If $W$ is an object of ${ }^{\perp} V$, then $W$ does not contain any projective summands. Then one can define the Auslander-Reiten translate $\tau(W)$ of $W$. From Auslander-Reiten duality (see properties (5), (6), (7) on pages 75-76 in 36. follows that $\tau(W)$ lies in the right perpendicular category. The Auslander-Reiten transform induces an equivalence of categories.

Theorem 2.28 (Theorem 2.3 of [38]). Suppose that $V$ is an exceptional representation of a quiver $Q$ without oriented cycles and with $n=\# Q_{0}$ vertices. Then $V^{\perp}$ (resp. $\left.{ }^{\perp} V\right)$ is equivalent to $\operatorname{Rep}_{K}\left(Q^{\prime}\right)$ where $Q^{\prime}$ is a quiver without oriented cycles such that $\# Q_{0}^{\prime}=n-1$.

Suppose we are in the situation of Theorem 2.28. The category $V^{\perp} \cong \operatorname{Rep}_{K}\left(Q^{\prime}\right)$ has exactly $n-1$ simple objects, say $E_{1}, E_{2}, \ldots, E_{n-1}$. Now $Q^{\prime}$ is the graph with vertices $1,2, \ldots, n-1$ and $r_{i, j}:=\operatorname{dim}_{\operatorname{Ext}_{Q^{\prime}}}\left(E_{i}, E_{j}\right)$ arrows from $i$ to $j$ for all $i, j$. We have

$$
\operatorname{Hom}_{Q^{\prime}}\left(E_{i}, E_{i}\right) \cong \operatorname{Hom}_{Q}\left(E_{i}, E_{i}\right) \cong K
$$

for all $i$. This shows that $E_{1}, \ldots, E_{n-1}$ are Schur representations. The category $V^{\perp}$ is closed under extensions, so every nontrivial extension of $E_{i}$ with itself in the category $\operatorname{Rep}(Q)$ would yield a nontrivial extension of $E_{i}$ with itself in the category $\operatorname{Rep}\left(Q^{\prime}\right)$. Since $\operatorname{Ext}_{Q^{\prime}}\left(E_{i}, E_{i}\right)=0$, we have $\operatorname{Ext}_{Q}\left(E_{i}, E_{i}\right)=0$. Therefore, $E_{1}, \ldots, E_{n-1}$ are exceptional representations for $Q$. Let $W$ be an object of $V^{\perp} \cong \operatorname{Rep}_{K}\left(Q^{\prime}\right)$. Suppose that, as a representation of $Q^{\prime}$, its dimension vector is $\alpha^{\prime}=$ $\left(\alpha_{1}^{\prime}, \ldots, \alpha_{n-1}^{\prime}\right)$. Then $W$ can be build up from extensions using $\alpha_{i}^{\prime}$ copies of $E_{i}$ for $i=1,2, \ldots, n-1$. This shows that the dimension vector of $W$, as a representation of $Q$ is equal to

$$
\alpha:=\sum_{i=1}^{n-1} \alpha_{i}^{\prime} \varepsilon_{i}
$$


where $\varepsilon_{i}=\underline{\operatorname{dim}}_{Q} E_{i}$, the dimension vector of $E_{i}$ seen as a representation of $Q$. Let us define

$$
I: \mathbb{N}^{Q_{0}^{\prime}} \cong \mathbb{N}^{n-1} \rightarrow \mathbb{N}^{Q_{0}}
$$

by

$$
I\left(\beta_{1}, \ldots, \beta_{n-1}\right)=\sum_{i=1}^{n-1} \beta_{i} \varepsilon_{i} .
$$

So if $W$ is a representation of $Q^{\prime}$ of dimension $\beta$, then $W$, viewed as a representation of $Q$, has dimension $I(\beta)$.

If $W$ and $Z$ are representations of $Q^{\prime}$, then

$$
\operatorname{Hom}_{Q}(W, Z) \cong \operatorname{Hom}_{Q^{\prime}}(W, Z)
$$

because $\operatorname{Rep}_{K}\left(Q^{\prime}\right)$ is a full subcategory of $\operatorname{Rep}_{K}(Q)$. Since $V^{\perp}$ is closed under extensions, we also have

From this follows that

$$
\operatorname{Ext}_{Q}(W, Z) \cong \operatorname{Ext}_{Q^{\prime}}(W, Z)
$$

$$
\operatorname{hom}_{Q^{\prime}}(\beta, \gamma)=\operatorname{hom}_{Q}(I(\beta), I(\gamma))
$$

and

$$
\operatorname{ext}_{Q^{\prime}}(\beta, \gamma)=\operatorname{ext}_{Q}(I(\beta), I(\gamma))
$$

Now we also get

$$
\begin{aligned}
\langle\beta, \gamma\rangle_{Q^{\prime}}=\operatorname{hom}_{Q^{\prime}}(\beta, \gamma)-\operatorname{ext}_{Q^{\prime}}(\beta, \gamma) & = \\
& =\operatorname{hom}_{Q}(I(\beta), I(\gamma))-\operatorname{ext}_{Q}(I(\beta), I(\gamma))=\langle I(\beta), I(\gamma)\rangle_{Q} .
\end{aligned}
$$

Lemma 2.29. Suppose that $\beta \in \mathbb{N}^{Q_{0}^{\prime}}$. Then $\beta$ is a Schur root (for $\left.Q^{\prime}\right)$ if and only if $I(\beta)$ is a Schur root (for $Q$ ).

Proof. If $W$ is a Schur representation of dimension $\beta$ for the quiver $Q^{\prime}$, then $W$ is also a Schur representation of dimension $I(\beta)$ as a representation of $Q$.

Conversely, suppose that $I(\beta)$ is a Schur root. Then a general representation of dimension $I(\beta)$ is a Schur representation. Since there exists a representation of dimension $I(\beta)$ in $V^{\perp}$, we have that a general representation of dimension $I(\beta)$ lies in $V^{\perp}$ (because $V \mapsto \operatorname{dim}_{\operatorname{Hom}_{Q}}(V, W)$ and $V \mapsto \operatorname{dim} \operatorname{Ext}_{Q}(V, W)$ are upper semi-continuous). A general representation of dimension $I(\beta)$, can be seen as a $\beta$-dimensional Schur representation for $Q^{\prime}$.

Theorem 2.30. If $\beta, \gamma \in \mathbb{N}_{0}^{\prime}$ and $\beta \perp \gamma$, then

$$
(I(\beta) \circ I(\gamma))_{Q}=(\beta \circ \gamma)_{Q^{\prime}}
$$

Proof. Choose $W \in \operatorname{Rep}\left(Q^{\prime}, \beta+\gamma\right)$ in general position. So $W$ has $(\beta \circ \gamma)_{Q^{\prime}}$ subrepresentations of dimension $\beta$. These subrepresentations correspond to $I(\beta)$-dimensional subrepresentations of $W$, seen as representations of $Q$. Suppose that $Z$ is an $I(\beta)$-dimensional subrepresentation of $W$. Since $Z$ is a subrepresentation of $W, \operatorname{Hom}_{Q}(V, W)=0$ implies $\operatorname{Hom}_{Q}(V, Z)=0$. Since $\langle\beta, \gamma\rangle=0$ we get $\operatorname{Ext}_{Q}(V, Z)=0$ as well. This implies that $Z$ lies in $V^{\perp}$, so $Z$ may be viewed as a representation of $Q^{\prime}$. As a representation of $Q, W$ has exactly $(\beta \circ \gamma)_{Q^{\prime}}$ subrepresentations. By Lemma 2.11 we obtain

$$
(I(\beta) \circ I(\gamma))_{Q} \geq(\beta \circ \gamma)_{Q^{\prime}}
$$

Choose $W \in \operatorname{Rep}(Q, I(\beta)+I(\gamma))$ in general position. Then $W$ has exactly $(I(\beta) \circ I(\gamma))_{Q}$ subrepresentations of dimension $I(\beta)$. We have $\operatorname{Hom}_{Q}(V, W)=\operatorname{Ext}_{Q}(V, W)=0$ (by semicontinuity) so $W$ lies 
in $V^{\perp}$. We may view $W$ as a representation of $Q^{\prime}$ of dimension $\beta+\gamma$. Again, the $I(\beta)$-dimensional subrepresentations of $W$, seen as a representation of $Q$ are exactly the $\beta$-dimensional subrepresentations of $W$, seen as a representation of $Q^{\prime}$. So as a representation of $Q^{\prime}, W$ has exactly

$$
(I(\beta) \circ I(\gamma))_{Q}
$$

subrepresentations of dimension $\beta$. Again, Lemma 2.11 implies that

$$
(I(\beta) \circ I(\gamma))_{Q} \leq(\beta \circ \gamma)_{Q^{\prime}}
$$

We conclude that

$$
(I(\beta) \circ I(\gamma))_{Q}=(\beta \circ \gamma)_{Q^{\prime}}
$$

2.7. Exceptional Sequences. We will introduce exceptional sequences and their basic properties. For more details, see [9, 37.

Definition 2.31. An exceptional sequence is a sequence $E_{1}, \ldots, E_{r}$ of exceptional representations such that $E_{i} \perp E_{j}$ for $i<j$.

Define $\varepsilon_{i}:=\underline{\operatorname{dim}} E_{i}$ for all $i$. The matrix $\left(\left\langle\varepsilon_{i}, \varepsilon_{j}\right\rangle\right)_{i, j}$ is lower triangular with 1's on the diagonal, and is therefore invertible. It follows that $\varepsilon_{1}, \ldots, \varepsilon_{r}$ are linearly independent, hence $r \leq n:=\# Q_{0}$.

Definition 2.32. An exceptional sequence $E_{1}, \ldots, E_{r}$ is called maximal or complete if $r=n$.

Lemma 2.33 (Lemma 1 of [9]). If $E_{1}, E_{2}, \ldots, E_{i}, E_{j}, E_{j+1}, \ldots, E_{n}$ is an exceptional sequence $(i<j)$ then there exist $E_{i+1}, \ldots, E_{j-1}$ such that $E_{1}, E_{2}, \ldots, E_{n}$ is an exceptional sequence.

In particular, every exceptional sequence $E_{1}, \ldots, E_{r}$ can be extended to a maximal exceptional sequence $E_{1}, \ldots, E_{n}$. To see this, consider the full subcategory of all representations $V$ such that

$$
E_{i} \perp V \text { for } i=1,2, \ldots, r \text {. }
$$

Let us denote this category by

$$
E_{1}^{\perp} \cap E_{2}^{\perp} \cap \cdots \cap E_{r}^{\perp}
$$

or simply $E^{\perp}$ where $E=\left(E_{1}, \ldots, E_{r}\right)$. Using Theorem 2.28 and induction on $r$ we see that this category $E^{\perp}$ is equivalent to the category of representations $\operatorname{Rep}\left(Q^{\prime}\right)$ of a quiver $Q^{\prime}$ with $n-r$ vertices and with no oriented cycles. Let $E_{r+1}, \ldots, E_{n}$ be the simple representations (pairwise non-isomorphic) in $E^{\perp}$ corresponding to the $n-r$ vertices of $Q^{\prime}$. We can order $E_{r+1}, \ldots, E_{n}$ in such way that $E_{j} \perp E_{k}$ for all $j, k$ with $r+1 \leq j<k \leq n$, because $Q^{\prime}$ has no oriented cycles. Then $E_{1}, \ldots, E_{n}$ is a (maximal) exceptional sequence. Lemma 2.33 is proved in a similar fashion (see [9]).

Definition 2.34. If $E_{1}, E_{2}, \ldots, E_{r}$ is an exceptional sequence, then we define $\mathcal{C}\left(E_{1}, \ldots, E_{r}\right)$ as the full subcategory of $\operatorname{Rep}(Q)$ which contains $E_{1}, \ldots, E_{r}$ and is closed under extensions, kernels of epimorphisms, and cokernels of monomorphisms.

Lemma 2.35 (Lemma 4 of [9]). If $E_{1}, \ldots, E_{n}$ is a maximal exceptional sequence, then $\mathcal{C}\left(E_{1}, \ldots, E_{r}\right)$ is equivalent to the category

$$
{ }^{\perp} E_{r+1} \cap \cdots \cap{ }^{\perp} E_{n}={ }^{\perp} F
$$

where $F=\left(E_{r+1}, \ldots, E_{n}\right)$.

Lemma 2.36. Suppose that $E_{1}, E_{2}, \ldots, E_{r}$ is an exceptional sequence, and $\operatorname{Hom}_{Q}\left(E_{i}, E_{j}\right)=0$ for all $i \neq j$. Then $E_{1}, \ldots, E_{r}$ are exactly all simple objects in $\mathcal{C}\left(E_{1}, E_{2}, \ldots, E_{r}\right)$. 
Proof. Let $\mathcal{D}\left(E_{1}, E_{2}, \ldots, E_{r}\right)$ be the the smallest full subcategory of $\operatorname{Rep}(Q)$ which contains $E_{1}, \ldots, E_{r}$ and which is closed under extensions. The objects of $\mathcal{D}\left(E_{1}, \ldots, E_{r}\right)$ are all representations which allow a filtration such that each factor is isomorphic to one of the representations $E_{1}, \ldots, E_{r}$. We claim that $\mathcal{D}\left(E_{1}, \ldots, E_{r}\right)=\mathcal{C}\left(E_{1}, \ldots, E_{r}\right)$. To show this, it suffices to show that the category $\mathcal{D}\left(E_{1}, \ldots, E_{r}\right)$ is closed under taking kernels of epimorphisms and taking cokernels of monomorphisms. We will show that $\mathcal{D}\left(E_{1}, \ldots, E_{r}\right)$ is closed under taking cokernels of monomorphisms. Dualizing the arguments one can then show that $\mathcal{D}\left(E_{1}, \ldots, E_{r}\right)$ is also closed under taking kernels of epimorphisms. Suppose that $\phi: V \rightarrow W$ is a monomorphism and $V, W$ are objects of $\mathcal{D}\left(E_{1}, \ldots, E_{r}\right)$. We have filtrations

$$
\begin{gathered}
V=F^{0}(V) \supset F^{1}(V) \supset \cdots \supset F^{s}(V)=\{0\} \\
W=F^{0}(W) \supset F^{1}(W) \supset \cdots \supset F^{t}(W)=\{0\}
\end{gathered}
$$

such that all quotients $F^{i}(V) / F^{i+1}(V), F^{i}(W) / F^{i+1}(W)$ are isomorphic to one of the representations $E_{1}, \ldots, E_{r}$.

The case $s=0$ is trivial. Suppose that $s=1$. Then we have that $V=F^{0}(V)$ is isomorphic to one of the representations $E_{1}, \ldots, E_{r}$. We prove the statement by induction on $t$. If $t=1$, then $\phi$ must be an isomorphism because $V$ and $W$ are simple. So $V / W=0$ and we are done. Suppose that $t>0$. Let $\psi$ be the composition $V \rightarrow W \rightarrow W / F^{1}(W)$. Suppose that $\psi=0$. Then $V$ is a subrepresentation of $F^{1}(W)$ By induction $F^{1}(W) / V$ is an object of $\mathcal{D}\left(E_{1}, \ldots, E_{r}\right)$, and $W / F^{1}(W)$ is also an object of $\mathcal{D}\left(E_{1}, \ldots, E_{r}\right)$. From the exact sequence

$$
0 \rightarrow F^{1}(W) / V \rightarrow W / V \rightarrow W / F^{1}(W) \rightarrow 0
$$

follows that $W / V$ is an object of $\mathcal{D}\left(E_{1}, \ldots, E_{r}\right)$.

Suppose that $\psi \neq 0$. Both $V$ and $W / F^{1}(W)$ are isomorphic to one of the $E_{1}, \ldots, E_{r}$. Because $V$ and $W / F^{1}(W)$ are simple, they are isomorphic to each other and to $E_{i}$ for some $i$. Since $E_{i}$ is exceptional and $\psi$ is nonzero, we must have that $\psi$ is an isomorphism. It follows that $V+F^{1}(W)=W$ and $V \cap F^{1}(W)=0$. But then

$$
F^{1}(W)=F^{1}(W) /\left(V \cap F^{1}(W)\right) \cong\left(F^{1}(W)+V\right) / V=W / V
$$

This shows that $W / V$ is an object of $\mathcal{D}\left(E_{1}, \ldots, E_{r}\right)$. We have proved the case $s=1$.

Suppose now that $s>1$. We will prove the theorem by induction on $s$. By the above, we now that $W / F^{s-1}(V)$ and $V / F^{s-1}(V)$ are objects of $\mathcal{D}\left(E_{1}, \ldots, E_{r}\right)$. From induction and the exact sequence

$$
0 \rightarrow V / F^{s-1}(V) \rightarrow W / F^{s-1}(V) \rightarrow W / V \rightarrow 0
$$

we conclude that $W / V$ also is an object of $\mathcal{D}\left(E_{1}, \ldots, E_{r}\right)$. We have proved that $\mathcal{D}\left(E_{1}, \ldots, E_{r}\right)=$ $\mathcal{C}\left(E_{1}, \ldots, E_{r}\right)$.

Since every object in $\mathcal{D}\left(E_{1}, \ldots, E_{r}\right)=\mathcal{C}\left(E_{1}, \ldots, E_{r}\right)$ has a subobject isomorphic to one of the representations $E_{1}, \ldots, E_{r}$, the only possible simple objects of $\mathcal{C}\left(E_{1}, \ldots, E_{r}\right)$ are $E_{1}, \ldots, E_{r}$. It is also easy to see that each $E_{i}$ is simple. Indeed, if $W$ is a proper subrepresentation of $E_{i}$, then $W$ has a proper subrepresentation isomorphic to $E_{k}$ for some $k \neq i$. But then $E_{k}$ is a proper subrepresentation of $E_{i}$ which contradicts the assumption that $\operatorname{Hom}_{Q}\left(E_{k}, E_{i}\right)=0$.

As we have noted before, there is a bijection between real Schur roots and exceptional representations. Suppose that $E_{1}, \ldots, E_{r}$ are exceptional representations and let $\varepsilon_{i}:=\underline{\operatorname{dim}} E_{i}$. We have seen that the orbit of $E_{i}$ in $\operatorname{Rep}\left(Q, \varepsilon_{i}\right)$ is dense. From this follows that

$$
\operatorname{dim} \operatorname{Hom}_{Q}\left(E_{i}, E_{j}\right)=\operatorname{hom}_{Q}\left(\varepsilon_{i}, \varepsilon_{j}\right)
$$

and

$$
\operatorname{dim} \operatorname{Ext}_{Q}\left(E_{i}, E_{j}\right)=\operatorname{ext}_{Q}\left(\varepsilon_{i}, \varepsilon_{j}\right)
$$

for all $i, j$. This allows us to give a more combinatorial definition of an exceptional sequence. 
Definition 2.37. A sequence of dimension vectors $\varepsilon_{1}, \ldots, \varepsilon_{r}$ is called an exceptional sequence if $\varepsilon_{1}, \ldots, \varepsilon_{r}$ are real Schur roots, and $\varepsilon_{i} \perp \varepsilon_{j}$ for all $i<j$.

So if $E_{1}, \ldots, E_{r}$ is an exceptional sequence of quiver representations, then $\varepsilon_{1}, \ldots, \varepsilon_{r}$ is an exceptional sequence of dimension vectors, where $\varepsilon_{i}=\underline{\operatorname{dim}} E_{i}$ for all $i$. Conversely, suppose that $\varepsilon_{1}, \ldots, \varepsilon_{r}$ is an exceptional sequence of dimension vector. Since $\varepsilon_{i}$ is a real Schur root, the exists a unique dense orbit in $\operatorname{Rep}\left(Q, \varepsilon_{i}\right)$. Let $E_{i}$ be a representation that lies in that orbit ( $E_{i}$ is unique op to isomorphism). Then $E_{1}, \ldots, E_{r}$ is an exceptional sequence of representations.

Theorem 2.38 (Theorem 4.1 of [39]). Suppose that $\alpha, \beta$ are Schur roots such that $\operatorname{ext}(\alpha, \beta)=0$. Then $\operatorname{hom}(\beta, \alpha)=0$ or $\operatorname{ext}(\beta, \alpha)=0$. Moreover, if both $\alpha$ and $\beta$ are imaginary, then $\operatorname{hom}(\beta, \alpha)=0$.

Theorem 2.39 (Embedding Theorem). Suppose that $\varepsilon_{1}, \ldots, \varepsilon_{r}$ is an exceptional sequence for the quiver $Q$ (without orientations). Suppose that $\left\langle\varepsilon_{i}, \varepsilon_{j}\right\rangle \leq 0$ for all $i>j$. We define a quiver $Q^{\prime}$ with vertices $Q_{0}^{\prime}=\{1,2, \ldots, r\}$. We draw $-\left\langle\varepsilon_{i}, \varepsilon_{j}\right\rangle$ arrows from $i$ to $j$ for all $i>j$. We define

$$
I: \mathbb{N}^{Q_{0}^{\prime}} \cong \mathbb{N}^{r} \rightarrow \mathbb{N}^{Q_{0}}
$$

by

$$
I\left(\beta_{1}, \ldots, \beta_{r}\right)=\sum_{i=1}^{r} \beta_{i} \varepsilon_{i}
$$

for all $\beta=\left(\beta_{1}, \ldots, \beta_{r}\right) \in \mathbb{N}^{Q_{0}^{\prime}} \cong \mathbb{N}^{r}$.

(a) For all $\beta, \gamma \in \mathbb{N}_{0}^{\prime}$ we have

$$
\begin{aligned}
\operatorname{hom}_{Q}(I(\beta), I(\gamma)) & =\operatorname{hom}_{Q^{\prime}}(\beta, \gamma), \\
\operatorname{ext}_{Q}(I(\beta), I(\gamma)) & =\operatorname{ext}_{Q^{\prime}}(\beta, \gamma), \\
\langle I(\beta), I(\gamma)\rangle_{Q} & =\langle\beta, \gamma\rangle_{Q^{\prime}} .
\end{aligned}
$$

(b) The dimension vector $\beta$ is a Schur root (for $\left.Q^{\prime}\right)$ if and only if $I(\beta)$ is a Schur root (for $Q$ ).

(c) For all $\beta, \gamma \in \mathbb{N}_{0}^{\prime}$ with $\beta \perp \gamma$ we have

$$
(I(\beta) \circ I(\gamma))_{Q}=(\beta \circ \gamma)_{Q^{\prime}}
$$

Proof. Let $E_{i}$ be a representation corresponding to the dense orbit in $\operatorname{Rep}\left(Q, \varepsilon_{i}\right)$. Then $E_{1}, E_{2}, \ldots, E_{r}$ is an exceptional sequence. For $i>j$ we have

$$
\left\langle\varepsilon_{i}, \varepsilon_{j}\right\rangle=\operatorname{hom}\left(\varepsilon_{i}, \varepsilon_{j}\right)-\operatorname{ext}\left(\varepsilon_{i}, \varepsilon_{j}\right) \leq 0
$$

Since $\operatorname{hom}\left(\varepsilon_{i}, \varepsilon_{j}\right)=0$ or $\operatorname{ext}\left(\varepsilon_{i}, \varepsilon_{j}\right)=0$ by Theorem 2.38, we must have hom $\left(\varepsilon_{i}, \varepsilon_{j}\right)=0$. It follows that $\operatorname{Hom}_{Q}\left(E_{i}, E_{j}\right)=0$ for $i>j$. From Lemma 2.36 we see that $E_{1}, E_{2}, \ldots, E_{r}$ are exactly all simple objects in $\mathcal{C}\left(E_{1}, \ldots, E_{r}\right)$. We can extend $E_{1}, \ldots, E_{r}$ to a maximal exceptional sequence $E_{1}, E_{2}, \ldots, E_{n}$ using Lemma 2.33 Now, we have equality

$$
\mathcal{C}\left(E_{1}, \ldots, E_{r}\right)={ }^{\perp} E_{r+1} \cap \cdots \cap{ }^{\perp} E_{n}
$$

by Lemma 2.35 .

Suppose that $r=n-1$. Then we are exactly in the situation of Theorem 2.28 We note that the definition of the quiver $Q^{\prime}$ and the map $I$ coincide with the definitions in the discussion after Theorem 2.28, In particular, we get (see (13), (14), (15))

and

$$
\begin{aligned}
\operatorname{hom}_{Q^{\prime}}(\beta, \gamma) & =\operatorname{hom}_{Q}(I(\beta), I(\gamma)) \\
\operatorname{ext}_{Q^{\prime}}(\beta, \gamma) & =\operatorname{ext}_{Q}(I(\beta), I(\gamma)) .
\end{aligned}
$$

$$
\langle\beta, \gamma\rangle_{Q^{\prime}}=\langle I(\beta), I(\gamma)\rangle_{Q}
$$


Lemma 2.29 implies that $\beta \in \mathbb{N}_{0}^{\prime}$ is a Schur root if and only if $I(\beta)$ is a Schur root. Finally, Theorem 2.30 implies that

$$
(\beta \circ \gamma)_{Q^{\prime}}=(I(\beta) \circ I(\gamma))_{Q}
$$

If $r<n$, the theorem can be proved in a similar way, using induction on $n-r$.

Theorem 2.39 allows us to "embed" the combinators of a quiver $Q$ ' into the combinators of another quiver $Q$, using exceptional sequences. This is a very useful tool. Of course, we would like to be able to construct such exceptional sequences. Crawley-Boevey defined an action of the braid group on the set of all maximal exceptional sequences and he proved that this action is transitive (see [9]).

So let us assume that $Q$ is a quiver without orientations and $n$ vertices. Assume that the vertices are labeled by $1,2, \ldots, n$. Since there are no oriented cycles, we can arrange the vertices in such way that for every arrow $i \rightarrow j$ in the quiver we have $i>j$. If $S_{i}$ is the simple representation corresponding to the vertex $i$, then $S_{1}, S_{2}, \ldots, S_{n}$ is a maximal exceptional sequence.

The braid group $\mathcal{B}_{n}$ is the group given by generators $s_{1}, s_{2}, \ldots, s_{n-1}$ and relations

$$
\begin{gathered}
s_{i} s_{j}=s_{j} s_{i}, \quad \text { if }|i-j| \geq 2 \text { and } \\
s_{i} s_{i+1} s_{i}=s_{i+1} s_{i} s_{i+1}
\end{gathered}
$$

for $i=1,2, \ldots, n-2$. Let $\mathcal{E}_{Q}$ be the set of all maximal exceptional sequences for $Q$. We will define the action of $\mathcal{B}_{n}$ on $\mathcal{E}_{Q}$ as follows. Suppose that $E_{1}, E_{2}, \ldots, E_{n}$ is a maximal exceptional sequence. Let $\varepsilon_{1}, \varepsilon_{2}, \ldots, \varepsilon_{n}$ be their dimension vectors. Then we define

$$
s_{i}\left(E_{1}, E_{2}, \ldots, E_{n}\right)=\left(E_{1}, \ldots, E_{i-1}, E_{i+1}^{\prime}, E_{i}, E_{i+2}, \ldots, E_{n}\right)
$$

where $E_{i+1}^{\prime}$ is the unique simple object in ${ }^{\perp} E_{i}$ within the subcategory $\mathcal{C}\left(E_{i}, E_{i+1}\right)$. Let $\varepsilon_{i+1}^{\prime}$ be the dimension vector of $E_{i+1}^{\prime}$. From Lemma 2.35 follows that $\mathcal{C}\left(E_{i}, E_{i+1}\right)=\mathcal{C}\left(E_{i+1}^{\prime}, E_{i}\right)$. Therefore, we have

$$
\mathbb{Z} \varepsilon_{i}+\mathbb{Z} \varepsilon_{i+1}=\mathbb{Z} \varepsilon_{i}+\mathbb{Z} \varepsilon_{i+1}^{\prime}
$$

It follows that $\varepsilon_{i+1}^{\prime}=\varepsilon_{i+1}+k \varepsilon_{i}$ or $\varepsilon_{i+1}^{\prime}=-\varepsilon_{i+1}+k \varepsilon_{i}$ for some $k \in \mathbb{Z}$. We know that $\left\langle\varepsilon_{i+1}^{\prime}, \varepsilon_{i}\right\rangle=0$. There can only be two possibilities, namely

$$
\varepsilon_{i+1}^{\prime} \in\left\{\varepsilon_{i+1}-\left\langle\varepsilon_{i+1}, \varepsilon_{i}\right\rangle \varepsilon_{i},-\left(\varepsilon_{i+1}-\left\langle\varepsilon_{i+1}, \varepsilon_{i}\right\rangle \varepsilon_{i}\right)\right\} .
$$

This uniquely determines $\varepsilon_{i+1}^{\prime}$ because only one of two vectors on the right can lie in $\mathbb{N}^{Q_{0}}$.

We also define

$$
s_{i}^{-1}\left(E_{1}, E_{2}, \ldots, E_{n}\right)=\left(E_{1}, \ldots, E_{i-1}, E_{i+1}, E_{i}^{\prime}, E_{i+2}, \ldots, E_{n}\right)
$$

where $E_{i}^{\prime}$ is the unique simple object in $E_{i+1}^{\perp}$ within the subcategory $\mathcal{C}\left(E_{i}, E_{i+1}\right)$. Let $\varepsilon_{i}^{\prime}=\underline{\operatorname{dim}} E_{i}^{\prime}$. One can prove that

$$
\varepsilon_{i}^{\prime} \in\left\{\varepsilon_{i}-\left\langle\varepsilon_{i}, \varepsilon_{i+1}\right\rangle \varepsilon_{i+1},-\left(\varepsilon_{i}-\left\langle\varepsilon_{i}, \varepsilon_{i+1}\right\rangle \varepsilon_{i+1}\right)\right\} .
$$

One can show that this gives a well-defined action of the braid group $\mathcal{B}_{n}$ on the set $\mathcal{E}_{Q}$ of maximal exceptional sequences.

Theorem 2.40 ([9]). The action of $\mathcal{B}_{n}$ on $\mathcal{E}_{Q}$ is transitive.

2.8. Stability and GIT-quotients. King studied in 24 moduli spaces for representations of quivers (and more generally, finite dimensional algebras). Here we will study the moduli space of a quiver $Q$ without oriented cycles. Suppose that $\sigma \in \Gamma^{\star}$ is a weight. A representation $V \in \operatorname{Rep}(Q, \alpha)$ is called $\sigma$-semi-stable if there exists a positive integer $m$ and a semi-invariant $f \in \operatorname{SI}(Q, \alpha)_{m \sigma}$ such that $f(V) \neq 0$. King gave the following nice characterization of $\sigma$-stable and $\sigma$-semi-stable representations.

Theorem 2.41 (Proposition 3.1 of [24]).

(1) A representation $V$ is $\sigma$-semi-stable if $\sigma(\underline{\operatorname{dim}} V)=0$ and $\sigma(\underline{\operatorname{dim}} W) \leq 0$ for all subrepresentations $W \subseteq V$. 
(2) representation $V$ is $\sigma$-stable if $\sigma(\underline{\operatorname{dim}} V)=0$ and $\sigma(\underline{\operatorname{dim}} W)<0$ for all nonzero, proper subrepresentations $W \subset V$.

In King's setup the inequalities go the other way. His convention for writing weights are opposite to ours.

Example 2.42. Suppose that $\sigma=0$. Then a representation is $\sigma$-stable if and only if it is simple. Every representation is $\sigma$-semi-stable.

In general, $\sigma$-stable representations are indecomposable. Indeed, if $V=V_{1} \oplus V_{2}$ for some nontrivial representations $V_{1}$ and $V_{2}$, then $0=\sigma(\underline{\operatorname{dim}} V)=\sigma\left(\underline{\operatorname{dim}} V_{1}\right)+\sigma\left(\underline{\operatorname{dim}} V_{2}\right)$, so $\sigma\left(\underline{\operatorname{dim}} V_{1}\right) \leq 0$ or $\sigma\left(\underline{\operatorname{dim}} V_{2}\right) \leq 0$. If $d$ is a positive integer, then a representation $V$ is $\sigma$-stable if and only if it is $(d \sigma)$-stable. The set of $\sigma$-semi-stable representations in $\operatorname{Rep}(Q, \alpha)$ is denoted by $\operatorname{Rep}(Q, \alpha)_{\sigma}^{\text {ss }}$. We will assume that $\operatorname{Rep}(Q, \alpha)_{\sigma}^{\mathrm{ss}}$ is nonempty. In other words $\operatorname{SI}(Q, \alpha)_{n \sigma} \neq 0$ for some positive integer $n$.

Recall that $\sigma$ defines a character $\operatorname{GL}(Q, \alpha) \rightarrow K^{\star}$. Let $\operatorname{GL}(Q, \alpha)_{\sigma}$ be the kernel of this character.

Lemma 2.43. Suppose that $\sigma$ is not divisible by the characteristic of $K$. The invariant ring of $K[\operatorname{Rep}(Q, \alpha)]$ with respect to $\operatorname{GL}(Q, \alpha)_{\sigma}$ is equal to

$$
K[\operatorname{Rep}(Q, \alpha)]^{\mathrm{GL}(Q, \alpha)_{\sigma}}=\bigoplus_{n \geq 0} \operatorname{SI}(Q, \alpha)_{n \sigma} .
$$

Proof. The inequality $\supseteq$ is easy to see. We will prove $\subseteq$. Suppose that $f \in K[\operatorname{Rep}(Q, \alpha)]^{\mathrm{GL}(Q, \alpha)_{\sigma}}$. Then in particular

$$
f \in \operatorname{SI}(Q, \alpha)=\bigoplus_{\tau \in \Gamma^{\star}} \operatorname{SI}(Q, \alpha)_{\tau}
$$

Write $f=\sum_{\tau} f_{\tau}$ with $f_{\tau} \in \operatorname{SI}(Q, \alpha)_{\tau}$ for all $\tau \in \Gamma^{\star}$. Suppose that $f_{\eta} \neq 0$. If $\eta$ is not an integer multiple of $\sigma$, then there exists an $A \in \operatorname{GL}(Q, \alpha)_{\sigma}$ such that $\eta(A) \neq 1$. But then

$$
\sum_{\tau} f_{\tau}=f=A \cdot f=\sum_{\tau} A \cdot f_{\tau}=\sum_{\tau} \tau(A) f_{\tau}
$$

so $f_{\eta}=\eta(A) f_{\eta}$ and $f_{\eta}=0$. So $f_{\eta}=0$ unless $\eta$ is an integer multiple of $\sigma$. This shows that $f \in \bigoplus_{n \in \mathbb{Z}} \operatorname{SI}(Q, \alpha)_{n \sigma}$. We have assumed that there exists a nonzero $t \in \operatorname{SI}(Q, \alpha)_{\sigma} \neq 0$ for some positive $n$. This implies that $\operatorname{SI}(Q, \alpha)_{-m \sigma}=0$ for all $m>0$, for if $u \in \operatorname{SI}(Q, \alpha)_{-m \sigma}$ then $u^{n} t^{m} \in$ $K[\operatorname{Rep}(Q, \alpha)]^{\mathrm{GL}(Q, \alpha)}=K$ and $u$ must be constant. But the only constant function in $\operatorname{SI}(Q, \alpha)_{-m \sigma}$ is 0 .

Let $X=\operatorname{Spec}(R)$ be the affine variety corresponding to the ring

$$
R=K[\operatorname{Rep}(Q, \alpha)]^{\mathrm{GL}(Q, \alpha)_{\sigma}}=\bigoplus_{n \in \mathbb{N}} \operatorname{SI}(Q, \alpha)_{n \sigma} .
$$

(Here Spec denotes the spectrum of maximal ideals.) The inclusion $R \subseteq K[\operatorname{Rep}(Q, \alpha)]$ corresponds to a a morphism

$$
\psi: \operatorname{Rep}(Q, \alpha) \rightarrow X
$$

This morphism is an affine quotient with respect to a reductive group. Such a quotient map is known to be surjective. Note that $\psi^{-1}(0)$ is exactly the complement of $\operatorname{Rep}(Q, \alpha)_{\sigma}^{\text {ss }}$ in $\operatorname{Rep}(Q, \alpha)$. Here $0 \in X$ is the point corresponding to the homogeneous maximal ideal $\mathfrak{m}=\bigoplus_{n>0} \operatorname{SI}(Q, \alpha)_{n \sigma}$. Define

$$
Y=\operatorname{Proj}\left(\bigoplus_{n \geq 0} \operatorname{SI}(Q, \sigma)_{n \sigma}\right) .
$$


There is a natural surjective map $X \backslash\{0\} \rightarrow Y$. So we have a diagram

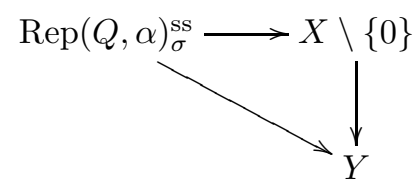

The map $\pi: \operatorname{Rep}(Q, \alpha)_{\sigma}^{\mathrm{ss}} \rightarrow Y$ is a GIT-quotient of $\operatorname{Rep}(Q, \alpha)$. Note that this quotient depends on $\sigma$. Again, the quotient $\pi$ is surjective.

\section{Stability}

3.1. Harder-Narasimhan and Jordan-Hölder filtrations. The notion of stability was compared (see [34, Section 3]) to the stability defined through a slope of two additive functions. In the quiver setting, the set of additive functions on the category can be identified with $\Gamma^{\star}$. Suppose that $\sigma, \tau \in \Gamma^{\star}$ and $\tau(\alpha)>0$ for all nonzero dimension vectors $\alpha$. The slope of a representation $V$ is defined by

$$
\mu(V):=\frac{\sigma(\underline{\operatorname{dim} V)}}{\tau(\underline{\operatorname{dim}} V)} .
$$

Definition 3.1. A representation $V$ is called $(\sigma: \tau)$-semi-stable if

$$
\mu(W) \leq \mu(V)
$$

for every proper subrepresentation $W \subseteq V$. It is called $(\sigma: \tau)$-stable if

$$
\mu(W)<\mu(V)
$$

for every proper subrepresentation $W \subset V$.

Rudakov showed (see [34, Lemma 3.2]) that the order $\prec$ defined by

$$
V \prec W \Leftrightarrow \mu(V)<\mu(W)
$$

and equivalence relation defined by

$$
V \asymp W \Leftrightarrow \mu(V)=\mu(W)
$$

defines a stability order in the sense of [34, Section 1].

Remark 3.2. The notions of $\sigma$-stability and $(\sigma: \tau)$-stability are closely related.

Suppose that $V$ is a representation of $Q$ and choose $\tau$ such that $\tau(\alpha)>0$ for every dimension vector $\alpha$. For example, define $\tau(\alpha):=\sum_{x \in Q_{0}} \alpha(x)$. If $V$ is $\sigma$-(semi-)stable, then $V$ is also $(\sigma: \tau)$ (semi-)stable.

Also, if $V$ is $(\sigma: \tau)$-(semi-)stable, and $\sigma(\underline{\operatorname{dim}} V) / \tau(\underline{\operatorname{dim}} V)=a / b$ where $a, b \in \mathbb{Z}$ and $b$ is positive, then $V$ is $(b \sigma-a \tau)$-(semi-)stable.

Theorems 2 and 3 from 34 give immediately the following results.

Proposition 3.3. With the assumptions of Definition [3.1] we have:

(1) (Harder-Narasimhan filtration) Every object $V$ has a filtration

$$
V=F_{H}^{0}(V) \supset F_{H}^{1}(V) \supset \ldots \supset F_{H}^{m}(V) \supset F_{H}^{m+1}(V)=0
$$

such that

(a) each factor $G_{H}^{i}(V)=F_{H}^{i}(V) / F_{H}^{i+1}(V)$ is $(\sigma: \tau)$-semi-stable;

$$
G_{H}^{0}(V) \prec G_{H}^{1}(V) \prec \ldots \prec G_{H}^{m}(V) .
$$

The filtration with properties (a), (b) is unique. 
(2) (Jordan-Hölder filtration) Every $(\sigma: \tau)$-semi-stable object $V$ has a filtration

$$
V=F_{J}^{0}(V) \supset F_{J}^{1}(V) \supset \ldots \supset F_{J}^{m}(V) \supset F_{J}^{m+1}(V)=0
$$

such that

(a) each factor $G_{J}^{i}(V)=F_{J}^{i}(V) / F_{J}^{i+1}(V)$ is $(\sigma: \tau)$-stable;

$$
G_{J}^{0}(V) \asymp G_{J}^{1}(V) \asymp \ldots \asymp G_{J}^{m}(V) .
$$

The set of factors $\left\{G_{J}^{i}(V)\right\}$ is uniquely determined by the properties (a), (b).

In part (2), the Jordan-Hölder filtration itself may not be unique.

Proposition 3.4. Suppose that a representation $V$ has the Harder-Narasimhan filtration

$$
V=F^{0}(V) \supset F^{1}(V) \supset \cdots \supset F^{m}(V) \supset F^{m+1}(V)=0 .
$$

If $W$ is a nonzero subrepresentation of $V$ then we have $\mu(W) \leq \mu\left(F^{m}(V)\right)$. If moreover $\mu(W)=$ $\mu\left(F^{m}(V)\right)$, then $W \subseteq F^{m}(V)$.

Proof. This follows immediately from Proposition 1.9 and Proposition 1.13 in [34.

Remark 3.5. Of course, by taking the Harder-Narasimhan filtration and using a Jordan-Hölder filtration on each quotient, one obtains a filtration of an object $V$ such that every quotient is $(\sigma: \tau)$ stable. Such a filtration we will call a Harder-Narasimhan-Jordan-Hölder filtration or HNJH-filtration for short.

Lemma 3.6. Let $Q$ be a quiver and $\alpha_{0}=\alpha, \alpha_{1}, \alpha_{2}, \ldots, \alpha_{m}, \alpha_{m+1}=0$ are dimension vectors. The set of all $V \in \operatorname{Rep}(Q, \alpha)$ of representations which allow a filtration

$$
V=F^{0}(V) \supset F^{1}(V) \supset \cdots \supset F^{m+1}(V)=0
$$

with $\underline{\operatorname{dim}} F^{i}(V)=\alpha_{i}$ is Zariski closed.

Proof. The proof goes exactly as in [39, Section 3].

The following Lemma follows immediately from the definition of $\sigma$-semi-stability.

Lemma 3.7. Suppose that $V$ is a $\sigma$-semi-stable representation, and $W$ is a subrepresentation with $\sigma(\underline{\operatorname{dim}} W)=0$, then $W$ is $\sigma$-semi-stable as well.

Lemma 3.8. Let $Q$ be a quiver and let $\alpha_{0}=\alpha, \alpha_{1}, \alpha_{2}, \ldots, \alpha_{m}, \alpha_{m+1}=0$ be dimension vectors.

(a) Define $U=U_{H N}\left(\alpha_{0}, \alpha_{1}, \ldots, \alpha_{m+1}\right)$ as the set of all representations $V \in \operatorname{Rep}(Q, \alpha)$ which have a Harder-Narasimhan filtration

$$
V=F^{0}(V) \supset F^{1}(V) \supset \cdots \supset F^{m+1}(V)=0
$$

with $\underline{\operatorname{dim}} F^{i}(V)=\alpha_{i}$ for all $i$. Then $U$ is locally closed.

(b) Define $U=U_{H N J H}\left(\alpha_{0}, \alpha_{1}, \ldots, \alpha_{m+1}\right)$ as the set of all representations $V \in \operatorname{Rep}(Q, \alpha)$ which have a HNJH-filtration

$$
V=F^{0}(V) \supset F^{1}(V) \supset \cdots \supset F^{m+1}(V)=0
$$

with $\underline{\operatorname{dim}} F^{i}(V)=\alpha_{i}$ for all $i$. Then $U$ is locally closed.

Proof. (a) Suppose that $U$ is not locally closed. This means that $\bar{U} \backslash U$ is not Zariski closed. Now $\bar{U} \backslash U$ is contained in the union of all sets of the form $U_{H N}\left(\beta_{0}, \ldots, \beta_{r+1}\right)$. Since there are only finitely many such sets, we can find $\beta_{0}, \beta_{1}, \ldots, \beta_{r+1}$ such that the Zariski closure of $U^{\prime} \cap(\bar{U} \backslash U)$ is not contained in $\bar{U} \backslash U$ for $U^{\prime}=U_{H N}\left(\beta_{0}, \ldots, \beta_{r+1}\right)$. So we have $\overline{U^{\prime}} \cap U \neq \emptyset$ and $U^{\prime} \cap \bar{U} \neq \emptyset$. 
Let $V \in U \cap \overline{U^{\prime}}$. Since $V \in U$, it has a Harder-Narasimhan filtration

$$
V=F_{H}^{0}(V) \supset F_{H}^{1}(V) \supset \ldots \supset F_{H}^{m}(V) \supset F_{H}^{m+1}(V)=0
$$

with $\underline{\operatorname{dim}} F_{H}^{i}(V)=\alpha_{i}$ for all $i$. Because $V \in \overline{U^{\prime}}$, Lemma 3.6 implies that it also has a filtration

$$
V=\widehat{F}^{0}(V) \supset \widehat{F}^{1}(V) \supset \ldots \supset \widehat{F}^{r}(V) \supset \widehat{F}^{r+1}(V)=0
$$

with $\underline{\operatorname{dim}} \widehat{F}^{i}(V)=\beta_{i}$ for all $i$.

Let $V^{\prime} \in \bar{U} \cap U^{\prime}$. Because $V \in U^{\prime}$, it has a Harder-Narasimhan filtration

$$
V^{\prime}=\widehat{F}_{H}^{0}\left(V^{\prime}\right) \supset \widehat{F}_{H}^{1}\left(V^{\prime}\right) \supset \ldots \supset \widehat{F}_{H}^{r}\left(V^{\prime}\right) \supset \widehat{F}_{H}^{r+1}\left(V^{\prime}\right)=0
$$

with $\underline{\operatorname{dim}} \widehat{F}_{H}^{i}\left(V^{\prime}\right)=\beta_{i}$. Because $V^{\prime} \in \bar{U}$, Lemma 3.6 implies that it has a filtration

$$
V^{\prime}=F^{0}\left(V^{\prime}\right) \supset F^{1}\left(V^{\prime}\right) \supset \ldots \supset F^{m}\left(V^{\prime}\right) \supset F^{m+1}\left(V^{\prime}\right)=0
$$

with $\underline{\operatorname{dim}} F^{i}\left(V^{\prime}\right)=\alpha_{i}$ for all $i$.

By induction on $i$ we will prove that $\widehat{F}^{r-i}(V)=F_{H}^{m-i}(V)$ and $F^{m-i}\left(V^{\prime}\right)=\widehat{F}_{H}^{r-i}\left(V^{\prime}\right)$.

We start the induction with the case $i=0$. Since $\widehat{F}^{r}(V)$ is a proper subrepresentation of $V$, Proposition 3.4 implies that $\mu\left(\beta_{r}\right) \leq \mu\left(\alpha_{m}\right)$. Similarly, because $F^{m}\left(V^{\prime}\right)$ is a subrepresentation of $V^{\prime}$,

$$
\mu\left(\alpha_{m}\right) \leq \mu\left(\beta_{r}\right)
$$

by Proposition 3.4. So we get $\mu\left(\alpha_{m}\right)=\mu\left(\beta_{r}\right)$. Proposition 3.4 shows that $\widehat{F}^{r}(V) \subseteq F_{H}^{m}(V)$ and $\widehat{F}_{H}^{r}\left(V^{\prime}\right) \supseteq F^{m}\left(V^{\prime}\right)$. We obtain $\beta_{r}=\alpha_{m}, \widehat{F}^{r}(V)=F_{N}^{m}(V)$ and $\widehat{F}_{H}^{r}\left(V^{\prime}\right)=F^{m}\left(V^{\prime}\right)$.

Suppose we already have shown that $\widehat{F}^{r-j}(V)=F_{H}^{m-j}(V)$ and $\widehat{F}_{H}^{r-j}\left(V^{\prime}\right)=F^{m-j}\left(V^{\prime}\right)$ for all $j<i$. Now $\widehat{F}^{r-i}(V) / \widehat{F}^{r-i+1}(V)$ is a subrepresentation of $V / F_{H}^{m-i+1}(V)$ and $F^{m-i}\left(V^{\prime}\right) / F^{m-i+1}\left(V^{\prime}\right)$ is a subrepresentation of $V^{\prime} / \widehat{F}_{H}^{r-i+1}\left(V^{\prime}\right)$. Similar reasoning as before shows that

$$
\mu\left(\beta_{r-i}-\beta_{r-i+1}\right)=\mu\left(\alpha_{m-i}-\alpha_{m-i+1}\right) .
$$

It follows that

$$
\widehat{F}^{r-i}(V) / \widehat{F}^{r-1+1}(V) \subseteq F_{H}^{m-i}(V) / F_{H}^{m-i+1}(V)
$$

and

$$
F^{m-i}\left(V^{\prime}\right) / F^{m-i+1}\left(V^{\prime}\right) \subseteq \widehat{F}_{H}^{r-i}\left(V^{\prime}\right) / \widehat{F}_{H}^{r-i+1}\left(V^{\prime}\right) .
$$

We get $\beta_{r-i}-\beta_{r-i+1}=\alpha_{m-i}-\alpha_{m-i+1}$ and equality in the previous inclusions. We conclude that $\beta_{r-i}=\alpha_{m-i}, F_{H}^{m-i}(V)=\widehat{F}^{r-i}(V)$ and $F^{m-i}\left(V^{\prime}\right)=\widehat{F}_{H}^{r-i}\left(V^{\prime}\right)$.

We have proved by induction that $\beta_{r-i}=\alpha_{m-i}$ for all $i$. In particular we get $r=m$. We have $U=U^{\prime}$. But then the Zariski closure of

$$
U^{\prime} \cap(\bar{U} \backslash U)=U \cap(\bar{U} \backslash U)=\emptyset
$$

is contained in $\bar{U} \backslash U$, contrary to our assumptions.

(b) Suppose that $U$ is not locally closed. Then $\bar{U} \backslash U$ is not Zariski closed. Now $\bar{U} \backslash U$ is contained in the union of all $U_{H N J H}\left(\beta_{0}, \ldots, \beta_{r+1}\right)$. Since this is only a finite union, there exist $\beta_{0}, \beta_{1}, \ldots, \beta_{r+1}$ such that the Zariski closure of $U^{\prime} \cap(\bar{U} \backslash U)$ is not contained in $\bar{U} \backslash U$, where $U^{\prime}=U_{H N J H}\left(\beta_{0}, \ldots, \beta_{r+1}\right)$. So we have $U^{\prime} \cap \bar{U} \neq \emptyset$ and $U \cap \overline{U^{\prime}} \neq \emptyset$. From (a) follows that elements of $U$ and of $U^{\prime}$ have the same dimensions in the Harder-Narasimhan filtrations, say

$$
U \subseteq U_{H N}\left(\gamma_{0}, \gamma_{1}, \ldots, \gamma_{t+1}\right) \supseteq U^{\prime}
$$

for some dimension vectors $\gamma_{0}=\alpha, \gamma_{1}, \ldots, \gamma_{t+1}=0$. It follows that

$$
\left\{\alpha_{0}, \ldots, \alpha_{m+1}\right\} \supseteq\left\{\gamma_{0}, \ldots, \gamma_{t+1}\right\}
$$

and

$$
\left\{\beta_{0}, \ldots, \beta_{r+1}\right\} \supseteq\left\{\gamma_{0}, \ldots, \gamma_{t+1}\right\} .
$$


Let $V^{\prime} \in U^{\prime} \cap \bar{U}$. Now $V^{\prime}$ has also a filtration $\left\{F^{i}\left(V^{\prime}\right)\right\}$ with dimensions $\alpha_{0}, \alpha_{1}, \ldots, \alpha_{m+1}$ by Lemma 3.6. This filtration is a refinement of the Harder-Narasimhan filtration of $V^{\prime}$. The quotients of this filtrations are $(\sigma: \tau)$-semi-stable (see Remark 3.2 and Lemma 3.7]). This means that the filtration $\left\{F^{i}\left(V^{\prime}\right)\right\}$ can be refined to a HNJH-filtration. We deduce that $m \leq r$. Considering a representation $V \in U \cap \overline{U^{\prime}}$ implies the reverse inequality. Therefore $m=r$. If $V^{\prime} \in U^{\prime} \cap \bar{U}$, then the filtration $\left\{F^{i}(V)\right\}$ with dimensions $\alpha_{0}, \alpha_{1}, \ldots, \alpha_{m+1}$ Any HNJH-filtration of $V^{\prime}$ has $r=m$ stable factors. This implies that the filtration $\left\{F^{i}(V)\right\}$ cannot be further refined to a HNJH-filtration. This means that it already is a HNJH-filtration. This shows that $U^{\prime} \cap \bar{U} \subseteq U$. This contradicts the fact that $U^{\prime} \cap(\bar{U} \backslash U) \neq \emptyset$.

Proposition 3.9. Let $Q$ be a quiver without oriented cycles, $\sigma, \tau(\tau(\alpha)>0$ for all nonzero dimension vectors) be weights and $\alpha$ be a dimension vector.

(a) There exists a nonempty open set $U \subseteq \operatorname{Rep}_{K}(Q, \alpha)$ such that for $V \in U$ the dimensions of the factors of the Harder-Narasimhan filtration with respect to $(\sigma: \tau)$ of $V$ are constant.

(b) There exists a nonempty open set $U \subseteq \operatorname{Rep}_{K}(Q, \alpha)$ such that for $V \in U$ the dimensions of the factors of the HNJH-filtration with respect to $(\sigma: \tau)$ of $V$ are constant.

(c) If a general representation of dimension $\alpha$ is $\sigma$-semi-stable, then there exists an nonempty open set $U \subseteq \operatorname{Rep}_{K}(Q, \alpha)$ such that for $V \in U$ the dimensions of the factors of a JordanHölder-filtration of $V$ are constant.

Proof.

(a) There exist dimension vectors $\alpha_{0}=\alpha, \alpha_{1}, \ldots, \alpha_{m}, \alpha_{m+1}=0$ such that

$$
U=U_{H N}\left(\alpha_{0}, \ldots, \alpha_{m+1}\right)
$$

lies dense in $\operatorname{Rep}(Q, \beta)$. Since $U$ is locally closed by Lemma 3.8 , we conclude that $U$ is open and dense in $\operatorname{Rep}(Q, \beta)$.

(b) goes similarly.

(c) Follows directly from (b) and Remark 3.2 .

\section{Lemma 3.10.}

(a) Suppose $\sigma \in \Gamma^{\star}$ is a weight and $V, W$ are $\sigma$-stable representations of the quiver. If $\phi: V \rightarrow W$ is a morphism then either $\phi=0$ or $\phi$ is an isomorphism.

(b) Suppose that $\sigma, \tau \in \Gamma^{\star}$ and $\tau(\alpha)>0$ for all nonzero dimension vectors $\alpha$. Assume that $V, W$ are $(\sigma: \tau)$-stable representations with $\mu(V) \geq \mu(W)$, where $\mu=\sigma / \tau$. If $\phi: V \rightarrow W$ is a morphism then either $\phi=0$ or $\phi$ is an isomorphism.

Proof. This follows immediately from [34, Theorem 1(d)].

3.2. The $\sigma$-stable decomposition. Let $Q$ be a quiver without oriented cycles.

Definition 3.11. Suppose that $\alpha$ is a dimension vector and $\sigma$ is a weight such that $\sigma(\alpha)=0$. A dimension vector $\alpha$ is called $\sigma$-(semi-)stable if a general representation of dimension $\alpha$ is $\sigma$-(semi)stable. The expression

$$
\alpha=\alpha_{1} \dot{+} \alpha_{2} \dot{+} \ldots \dot{+} \alpha_{s}
$$

is called the $\sigma$-stable decomposition of a $\sigma$-semi-stable dimension vector $\alpha$ if a general representation $V$ of dimension $\alpha$ has a Jordan-Hölder filtration with factors of dimension $\alpha_{1}, \alpha_{2}, \ldots, \alpha_{s}$ (in some order).

Proposition 3.12. A dimension vector $\alpha$ is a Schur root if and only if $\alpha$ is $\sigma$-stable for some weight $\sigma$. 
Proof. If $\alpha$ is $\sigma$-stable, then a general $\alpha$-dimensional representation is $\sigma$-stable, hence indecomposable. Conversely, if $\alpha$ is a Schur root, then $\alpha$ is $\sigma$-stable where

$$
\sigma=\langle\alpha, \cdot\rangle-\langle\cdot, \alpha\rangle \text {. }
$$

This follows from [39, Theorem 6.1].

Definition 3.13. Suppose that $\alpha$ is a dimension vector. Let $\sigma, \tau$ be weights. such that $\tau$ is positive on nonzero dimension vectors. A dimension vector $\alpha$ is called $(\sigma: \tau)$-stable if a general representation of dimension $\alpha$ is $(\sigma: \tau)$-stable. The expression

$$
\alpha=\alpha_{1} \dot{+} \alpha_{2} \dot{+} \ldots \dot{+} \alpha_{s}
$$

is called the $(\sigma: \tau)$-stable decomposition of $\alpha$ if a general representation $V$ of dimension $\alpha$ has a HNJH-filtration with factors of dimension $\alpha_{1}, \alpha_{2}, \ldots, \alpha_{s}$ (in some order).

Lemma 3.14. Let $\sigma, \tau$ be weights such that $\tau$ is positive on nonzero dimension vectors. Suppose that $\alpha \in \mathbb{N}^{Q_{0}}$ has $(\sigma: \tau)$-stable decomposition

$$
\alpha_{1} \dot{+} \alpha_{2} \dot{+} \cdots \dot{+} \alpha_{s} .
$$

For any indices $i_{1}<\cdots<i_{r}$ the $(\sigma: \tau)$-stable decomposition of $\beta=\alpha_{i_{1}}+\alpha_{i_{2}}+\cdots+\alpha_{i_{r}}$ is

$$
\alpha_{i_{1}} \dot{+} \alpha_{i_{2}} \dot{+} \cdots \dot{+} \alpha_{i_{r}} .
$$

Proof. After rearranging $\alpha_{1}, \ldots, \alpha_{s}$ we may assume that

$$
U_{\alpha}:=U_{H N J H}\left(\alpha_{1}+\cdots+\alpha_{s}, \alpha_{1}+\cdots+\alpha_{s-1}, \ldots, \alpha_{1}, 0\right)
$$

lies dense in $\operatorname{Rep}(Q, \alpha)$. By Lemma $3.8 U_{\alpha}$ is locally closed. Therefore, $U_{\alpha}$ is dense and open.

Let $j_{1}<j_{2}<\cdots<j_{s-r}$ such that

$$
\{1,2, \ldots, s\}=\left\{i_{1}, \ldots, i_{r}\right\} \cup\left\{j_{1}, \ldots, j_{s-r}\right\} .
$$

Then we have

$$
\alpha-\beta=\alpha_{j_{1}}+\cdots+\alpha_{j_{s-r}}
$$

Suppose that the $(\sigma: \tau)$-stable decomposition of $\beta$ is

$$
\beta_{1} \dot{+} \beta_{2} \dot{+} \cdots \dot{+} \beta_{t} .
$$

Choose $V_{i} \in \operatorname{Rep}\left(Q, \alpha_{i}\right)(\sigma: \tau)$-stable for $i=1,2, \ldots, s$. By Lemma 3.8, there exists an open dense set $U_{\beta} \subseteq \operatorname{Rep}(Q, \beta)$ and dimension vectors $\gamma_{0}=\alpha, \gamma_{1}, \ldots, \gamma_{l+1}=0$ such that

$$
V^{\prime} \oplus V_{j_{1}} \oplus \cdots \oplus V_{j_{s-r}} \in U_{\alpha}^{\prime}:=U_{H N J H}\left(\gamma_{0}, \ldots, \gamma_{l+1}\right)
$$

for all $V^{\prime} \in U_{\beta}$. Note that

is a rearrangement of

$$
\gamma_{0}-\gamma_{1}, \gamma_{1}-\gamma_{2}, \ldots, \gamma_{l}-\gamma_{l+1}
$$

We have

$$
\beta_{1}, \ldots, \beta_{t}, \alpha_{j_{1}}, \ldots, \alpha_{j_{s-r}}
$$

$$
V_{1} \oplus V_{2} \oplus \cdots \oplus V_{s} \in \overline{U_{\beta} \oplus V_{j_{1}} \oplus \cdots \oplus V_{j_{s-r}}} \subseteq \overline{U_{\alpha}^{\prime}} .
$$

This shows that $\overline{U_{\alpha}^{\prime}} \cap U_{\alpha} \neq \emptyset$. Since $U_{\alpha}$ is open, we have $U_{\alpha}^{\prime} \cap U_{\alpha} \neq \emptyset$. Therefore $U_{\alpha}^{\prime}=U_{\alpha}$. Hence

$$
\alpha_{1}, \alpha_{2}, \ldots, \alpha_{r}
$$

is a rearrangement of

$$
\alpha_{j_{1}}, \ldots, \alpha_{j_{r-s}}, \beta_{1}, \ldots, \beta_{t}
$$

and

$$
\beta_{1}, \ldots, \beta_{t}
$$


is a rearrangement of

$$
\alpha_{i_{1}}, \ldots, \alpha_{i_{r}} .
$$

Corollary 3.15. Let $\sigma$ be a weight and $\alpha$ be a $\sigma$-semi-stable dimension vector such that $\sigma(\alpha)=0$. Suppose that $\alpha \in \mathbb{N}^{Q_{0}}$ has $\sigma$-stable decomposition

$$
\alpha_{1} \dot{+} \alpha_{2} \dot{+} \cdots \dot{+} \alpha_{s} \text {. }
$$

For any indices $i_{1}<\cdots<i_{r}$ the $\sigma$-stable decomposition of $\beta=\alpha_{i_{1}}+\alpha_{i_{2}}+\cdots+\alpha_{i_{r}}$ is

$$
\alpha_{i_{1}} \dot{+} \alpha_{i_{2}} \dot{+} \cdots \dot{+} \alpha_{i_{r}} .
$$

Proof. This follows from Lemma 3.14 and Remark 3.2 .

Proposition 3.16. Suppose that $\alpha$ is a $\sigma$-stable dimension vector and $p \in \mathbb{N}$. Then the $\sigma$-stable decomposition of $p \alpha$ is

$$
\{p \alpha\}:= \begin{cases}p \cdot \alpha:=\underbrace{\alpha \dot{+} \cdots \dot{+} \alpha}_{p} & \text { if } \alpha \text { is real or isotropic } \\ p \alpha & \text { if } \alpha \text { is imaginary and nonisotropic. }\end{cases}
$$

Proof. Suppose that the $\sigma$-stable decomposition of $p \alpha$ is

$$
\gamma_{1} \dot{+} \gamma_{2} \dot{+} \cdots \dot{+} \gamma_{r}
$$

Let $W \in \operatorname{Rep}(Q, \alpha)$ be a $\sigma$-stable representation. Then $V=W^{p}=W \oplus \cdots \oplus W$ is $\sigma$-semi-stable and it has a filtration with factors of dimensions $\gamma_{1}, \ldots, \gamma_{r}$ (see Lemma 3.6). This filtration can be refined to a Jordan-Hölder filtration of $V$ whose factors are all isomorphic to the $\alpha$-dimensional representation $W$. This shows that $\gamma_{1}, \ldots, \gamma_{r}$ are multiples of $\alpha$. If $\alpha$ is real or isotropic, then the canonical decomposition of $p \alpha$ is $\alpha^{\oplus p}$. In particular, a general representation $Z$ of dimension $p \alpha$ has a filtration $\left\{F^{i}(Z)\right\}$ with factors of dimension $\alpha$. This filtration can be refined to a Jördan Hölder filtration. But since the factors of the Jördan Hölder filtration have dimensions that are multiples of $\alpha$, the filtration $\left\{F^{i}(Z)\right\}$ is already a Jordan-Hölder filtration. Therefore $r=p$ and $\gamma_{1}, \ldots, \gamma_{r}=\alpha$.

Suppose $\alpha$ is imaginary and not isotropic. Then $\gamma_{i} \hookrightarrow p \alpha$ for some $i$. Assume that $\gamma_{i}=q \alpha$. Then $q \alpha \hookrightarrow p \alpha$, so $\operatorname{ext}_{Q}(q \alpha,(p-q) \alpha)=0$. It follows that

$$
q(p-q)\langle\alpha, \alpha\rangle=\langle q \alpha,(p-q) \alpha\rangle \geq 0 .
$$

Since $\langle\alpha, \alpha\rangle<0$, we must have $p=q$. It follows that $i=1$ and $\gamma_{1}=p \alpha$.

Proposition 3.17. If the $\sigma$-stable decomposition of $\alpha$ is

$$
\alpha=\alpha_{1} \dot{+} \alpha_{2} \dot{+} \cdots \dot{+} \alpha_{s}
$$

then the $\sigma$-stable decomposition of $p \alpha$ is

$$
p \alpha=\left\{p \alpha_{1}\right\} \dot{+}\left\{p \alpha_{2}\right\} \dot{+} \cdots \dot{+}\left\{p \alpha_{s}\right\}
$$

for every positive integer $p$.

Proof. If $\beta \hookrightarrow \alpha$ then $\operatorname{ext}_{Q}(\beta, \alpha-\beta)=0$. It follows that $\operatorname{ext}_{Q}(p \alpha, p(\alpha-\beta))=0$ and $m \beta \hookrightarrow m \alpha$. Suppose that $V \in \operatorname{Rep}(Q, p \alpha)$. Then $V$ has a filtration with factors $V_{1}, V_{2}, \ldots, V_{s}$ of dimensions $p \alpha_{1}, \ldots, p \alpha_{s}$. From Proposition 3.16 and Lemma 3.6 follows that if $\alpha_{i}$ is isotropic or real, then each $V_{i}$ has a filtration such that all quotients have dimension $\alpha_{i}$. This shows that $V$ has a filtration $\left\{F^{i}(V)\right\}$ such that the dimensions of the factors are exactly all dimensions appearing on the right-hand side of (16). Assume now that $V$ is a general representation of dimension $p \alpha$. Then all factors of the filtration $\left\{F^{i}(V)\right\}$ are $\sigma$-semi-stable. There exists a Jordan-Hölder filtration $\left\{\left(F^{\prime}\right)^{i}(V)\right\}$ which is a refinement of the filtration $\left\{F^{i}(V)\right\}$. If $W$ is any representation of dimension $p \alpha$, then $W$ has a filtration $\left\{\left(F^{\prime}\right)^{i}(W)\right\}$ 
such that $\underline{\operatorname{dim}}\left(F^{\prime}\right)^{i}(W)=\underline{\operatorname{dim}}\left(F^{\prime}\right)^{i}(V)$ by Lemma 3.6. Take now $W=W_{1} \oplus W_{2} \oplus \cdots \oplus W_{s}$ with $W_{1}, \ldots, W_{s}$ in general position of dimension $p \alpha_{1}, \ldots, p \alpha_{s}$. The filtration $\left\{\left(F^{\prime}\right)^{i}(W)\right\}$ can be refined to a Jordan-Hölder filtration of $W$. But we know that the dimensions of the factors of the Jordan-Hölder filtration of $W$ are exactly the dimensions appearing on the right-hand side of (16) by Proposition 3.16. It follows that the filtrations $\left\{F^{i}(W)\right\}$ and $\left\{\left(F^{\prime}\right)^{i}(W)\right\}$ are the same, so the filtrations $\left\{F^{i}(V)\right\}$ and $\left\{\left(F^{\prime}\right)^{i}(V)\right\}$ are the same. This shows that the factors of the Jordan-Hölder filtration of $V$ are exactly given by the dimensions on the right-hand side of (16).

Proposition 3.18. Let $Q$ be a quiver without oriented cycles, $\sigma$ be a weight and $\alpha$ be a $\sigma$-semi-stable dimension vector. Let

$$
\alpha=c_{1} \cdot \alpha_{1} \dot{+} c_{2} \cdot \alpha_{2} \dot{+} \cdots \dot{+} c_{s} \cdot \alpha_{s}
$$

be the $\sigma$-stable decomposition where the $\alpha_{i}$ are distinct. Then we have

(a) All $\alpha_{i}$ are Schur roots;

(b) if $c_{i}>1$ then $\alpha_{i}$ must be a real or an isotropic root;

(c) $\operatorname{hom}_{Q}\left(\alpha_{i}, \alpha_{j}\right)=0$ if $i \neq j$;

(d) after rearranging one may assume that $\alpha_{i} \Perp \alpha_{j}$ for all $i<j$.

Proof.

(a) If general representations of dimension $\alpha_{i}$ are decomposable, then a general representation of dimension $\alpha_{i}$ is not $\sigma$-stable. Since a general representation of dimension $\alpha_{i}$ has a non-trivial JordanHölder filtration, every representation of dimension $\alpha_{i}$ has a nontrivial Jordan-Hölder filtration by Lemma 3.6] It follows that there are no $\sigma$-stable representations in dimension $\alpha_{i}$.

(b) If $c_{i} \geq 2$, then by Corollary 3.15, the $\sigma$-stable decomposition of $2 \alpha_{i}$ is $\alpha_{i} \dot{+} \alpha_{i}$. In particular we have $\alpha_{i} \hookrightarrow 2 \alpha_{i}$, so $\operatorname{ext}_{Q}\left(\alpha_{i}, \alpha_{i}\right)=0$ and $\left\langle\alpha_{i}, \alpha_{i}\right\rangle \geq 0$.

(c) Let $V_{i}$ and $V_{j}$ be $\sigma$-stable representations of dimensions $\alpha_{i}$ and $\alpha_{j}$ respectively. From Lemma 3.10 follows that any nonzero homomorphism between $V_{i}$ and $V_{j}$ must be an isomorphism. Since $\alpha_{i} \neq \alpha_{j}$, we have $\operatorname{Hom}_{Q}\left(V_{i}, V_{j}\right)=0$ and therefore $\operatorname{hom}_{Q}\left(\alpha_{i}, \alpha_{j}\right)=0$.

(d) Suppose that there exists an $r$-cycle:

$$
\operatorname{ext}_{Q}\left(\alpha_{i_{1}}, \alpha_{i_{2}}\right) \neq 0, \operatorname{ext}_{Q}\left(\alpha_{i_{2}}, \alpha_{i_{3}}\right) \neq 0, \ldots, \operatorname{ext}_{Q}\left(\alpha_{i_{r-1}}, \alpha_{i_{r}}\right) \neq 0, \operatorname{ext}_{Q}\left(\alpha_{i_{r}}, \alpha_{i_{1}}\right) \neq 0 .
$$

We assume that $r \geq 2$ is minimal such that an $r$-cycle exists. After rearranging $\alpha_{1}, \alpha_{2}, \ldots, \alpha_{r}$ we may assume that

$$
\operatorname{ext}_{Q}\left(\alpha_{1}, \alpha_{2}\right) \neq 0, \operatorname{ext}_{Q}\left(\alpha_{2}, \alpha_{3}\right) \neq 0, \ldots, \operatorname{ext}_{Q}\left(\alpha_{r-1}, \alpha_{r}\right) \neq 0, \operatorname{ext}_{Q}\left(\alpha_{r}, \alpha_{1}\right) \neq 0 .
$$

Also, by the minimality of $r$, $\operatorname{ext}_{Q}\left(\alpha_{i}, \alpha_{j}\right)=0$ if $1 \leq i, j \leq r$, unless $j=i$ or $j \equiv i+1(\bmod r)$. The $\sigma$-stable decomposition of $\beta=\alpha_{1}+\alpha_{2}+\cdots+\alpha_{r}$ is

$$
\alpha_{1} \dot{+} \alpha_{2} \dot{+} \cdots \dot{+} \alpha_{r}
$$

by Corollary 3.15. We have $\alpha_{i} \hookrightarrow \beta$ for some $i$, and after cyclic relabeling we may assume that $\alpha_{1} \hookrightarrow \beta$, so $\operatorname{ext}_{Q}\left(\alpha_{1}, \beta-\alpha_{1}\right)=0$. If $3 \leq i \leq r$, then we have $\operatorname{ext}_{Q}\left(\alpha_{i}, \alpha_{2}\right)=0$ by minimality of $r$. It follows that $\operatorname{ext}_{Q}\left(\alpha_{3}+\alpha_{4}+\cdots+\alpha_{r}, \alpha_{2}\right)=0$ or equivalently, $\beta-\alpha_{1} \rightarrow \alpha_{2}$. Consider an exact sequence

$$
0 \rightarrow V^{\prime} \rightarrow V \rightarrow V^{\prime \prime} \rightarrow 0
$$

with $V^{\prime}, V, V^{\prime \prime}$ of dimension $\beta-\alpha_{1}-\alpha_{2}, \beta-\alpha_{1}, \alpha_{2}$ respectively, and $V$ is in general position. Let $W$ be a general representation of dimension $\alpha_{1}$ and apply the functor $\operatorname{Hom}_{Q}(W, \cdot)$ to (17) to obtain a long exact sequence

$$
\cdots \rightarrow \operatorname{Ext}_{Q}\left(W, V^{\prime}\right) \rightarrow \operatorname{Ext}_{Q}(W, V) \rightarrow \operatorname{Ext}_{Q}\left(W, V^{\prime \prime}\right) \rightarrow 0 .
$$

Since $\operatorname{Ext}_{Q}(W, V)=0$, we have $\operatorname{Ext}_{Q}\left(W, V^{\prime \prime}\right)=0$. It follows that $\operatorname{ext}_{Q}\left(\alpha_{1}, \alpha_{2}\right)=0$. Contradiction.

Therefore, there are no $r$-cycles. So it is possible to rearrange $\alpha_{1}, \ldots, \alpha_{s}$ such that $\operatorname{ext}_{Q}\left(\alpha_{i}, \alpha_{j}\right)=0$ for all $i<j$. Together with $\operatorname{hom}_{Q}\left(\alpha_{i}, \alpha_{j}\right)=0$ by (c) we get $\alpha_{i} \perp \alpha_{j}$ for $i<j$. 
The number $p=\alpha_{i} \circ \alpha_{j}$ is finite and nonzero. We would like to show that $p=1$. Suppose that $p \geq 2$. Let $V$ be a general representation of dimension $\alpha_{i}+\alpha_{j}$. Then $V$ has $p \geq 2$ subrepresentations of dimension $\alpha_{i}$ (see Theorem 2.10). Let $V_{1}$ and $V_{2}$ be two distinct subrepresentations of $V$ of dimension $\alpha_{i}$. The $\sigma$-stable decomposition of $\alpha_{i}+\alpha_{j}$ is $\alpha_{i} \dot{+} \alpha_{j}$ by Corollary 3.15, This implies that $V_{1}, V_{2}, V / V_{1}, V / V_{2}$ are $\sigma$-stable. Let $\varphi: V_{1} \rightarrow V / V_{2}$ be the composition $V_{1} \rightarrow V \rightarrow V / V_{2}$. Since $V_{1}$ and $V_{2}$ are distinct of the same dimension, $\varphi$ is not equal the 0 map. Since $V_{1}, V / V_{2}$ are $\sigma$-stable $\varphi$ must be an isomorphism. This implies that $\alpha_{i}=\alpha_{j}$. Contradiction. So we conclude that $p=\alpha_{i} \circ \alpha_{j}=1$ and $\alpha_{i} \Perp \alpha_{j}$.

Corollary 3.19. Let $Q$ be a quiver without oriented cycles, $\sigma, \tau$ two weights with $\tau$ nonnegative on nonzero dimension vectors, and let $\alpha$ be a dimension vector. Let

$$
\alpha=c_{1} \cdot \alpha_{1} \dot{+} c_{2} \cdot \alpha_{2} \dot{+} \cdots \dot{+} c_{s} \cdot \alpha_{s}
$$

be the $(\sigma: \tau)$-stable decomposition where the $\alpha_{i}$ are distinct.

(a) All $\alpha_{i}$ are Schur roots;

(b) if $c_{i}>1$ then $\alpha_{i}$ must be a real root or an isotropic root;

(c) after rearranging one can assume that $\alpha_{i} \Perp \alpha_{j}$ for all $i<j$.

Proof. (a) and (b) follow from Proposition 3.18 (a), (b). Let $\mu=\sigma / \tau$. We may assume that $\mu\left(\alpha_{i}\right) \geq$ $\mu\left(\alpha_{j}\right)$ for all $i<j$. We have $\operatorname{hom}_{Q}\left(\alpha_{i}, \alpha_{j}\right)=0$ for $i<j$ by Lemma 3.10(b). Suppose that $\mu\left(\alpha_{i}\right)>$ $\mu\left(\alpha_{j}\right)$. The $(\sigma: \tau)$-stable decomposition of $\alpha_{i}+\alpha_{j}$ is $\alpha_{i}+\alpha_{j}$ (see Lemma 3.14). We have either $\alpha_{i} \hookrightarrow \alpha_{i}+\alpha_{j}$ or $\alpha_{j} \hookrightarrow \alpha_{i}+\alpha_{j}$. Because $\mu\left(\alpha_{i}\right)>\mu\left(\alpha_{j}\right), \alpha_{i}$ (and not $\alpha_{j}$ ) is the dimension of the submodule in the Harder-Narasimhan filtration of $\alpha_{i}+\alpha_{j}$, so $\alpha_{i} \hookrightarrow \alpha_{i}+\alpha_{j}$. This shows that $\operatorname{ext}_{Q}\left(\alpha_{i}, \alpha_{j}\right)=0$, and therefore $\alpha_{i} \perp \alpha_{j}$. Since the Harder-Narasimhan filtration is unique, a general representation of $\alpha_{i}+\alpha_{j}$ has a unique $\alpha_{i}$-dimensional subrepresentation. We conclude that $\alpha_{i} \Perp \alpha_{j}$ by Theorem 2.10 .

We can arrange $\alpha_{1}, \alpha_{2}, \ldots, \alpha_{s}$ such that $\mu\left(\alpha_{1}\right) \geq \mu\left(\alpha_{2}\right) \geq \cdots \geq \mu\left(\alpha_{s}\right)$. Using Proposition 3.18(d) and Remark 3.2 we see that we can even rearrange $\alpha_{1}, \ldots, \alpha_{s}$ such that $\alpha_{i} \Perp \alpha_{j}$ for all $i<j$.

Theorem 3.20. Suppose that $\sigma$ is an indivisible weight. If

$$
\alpha=c_{1} \cdot \alpha_{1} \dot{+} c_{2} \cdot \alpha_{2} \dot{+} \cdots \dot{+} c_{r} \cdot \alpha_{r}
$$

is the $\sigma$-stable decomposition of $\alpha$, then there exists an isomorphism

$$
\operatorname{SI}(Q, \alpha)_{m \sigma} \cong S^{c_{1}}\left(\operatorname{SI}\left(Q, \alpha_{1}\right)_{m \sigma}\right) \otimes S^{c_{2}}\left(\operatorname{SI}\left(Q, \alpha_{2}\right)_{m \sigma}\right) \otimes \cdots \otimes S^{c_{r}}\left(\operatorname{SI}\left(Q, \alpha_{r}\right)_{m \sigma}\right)
$$

Proof. First we assume that the base field $K$ has characteristic 0 . Let

$$
X:=\operatorname{Rep}\left(Q, \alpha_{1}\right)^{c_{1}} \oplus \operatorname{Rep}\left(Q, \alpha_{2}\right)^{c_{2}} \oplus \cdots \oplus \operatorname{Rep}\left(Q, \alpha_{r}\right)^{c_{r}} .
$$

We have a natural embedding

$$
\varphi: X \hookrightarrow \operatorname{Rep}(Q, \alpha) .
$$

Let $\operatorname{GL}(Q, \alpha)_{\sigma}$ be the kernel of $\sigma$, where $\sigma$ is interpreted as a multiplicative character $\operatorname{GL}(Q, \alpha) \rightarrow K^{\star}$. Let $G$ be the stabilizer of $X$ within $\operatorname{GL}(Q, \alpha)_{\sigma}$. This group $G$ is isomorphic to the intersection of

$$
\left(\Sigma_{c_{1}} \ltimes \mathrm{GL}\left(Q, \alpha_{1}\right)^{c_{1}}\right) \times\left(\Sigma_{c_{2}} \ltimes \mathrm{GL}\left(Q, \alpha_{2}\right)^{c_{2}}\right) \times \cdots \times\left(\Sigma_{c_{r}} \ltimes \mathrm{GL}\left(Q, \alpha_{r}\right)^{c_{r}}\right)
$$

and $\operatorname{GL}(Q, \alpha)_{\sigma}$. Here $\Sigma_{c}$ is the symmetric group on $c$ elements. Let $\pi_{X}: X \rightarrow X / / G$ and $\pi$ : $\operatorname{Rep}(Q, \alpha) \rightarrow \operatorname{Rep}(Q, \alpha) / / \operatorname{GL}(Q, \alpha)_{\sigma}$ be the categorical quotients. The embedding $\varphi$ induces a morphism between categorical quotients

$$
\psi: X / / G \rightarrow \operatorname{Rep}(Q, \alpha) / / \operatorname{GL}(Q, \alpha)_{\sigma} .
$$

We will show that $\psi$ is an isomorphism. 
First we will show that $\psi$ is dominant. Let $V \in \operatorname{Rep}(Q, \alpha)$ be a general representation and suppose $V$ is $\sigma$-semi-stable, i.e., $\pi(V) \neq 0$. Let

$$
0=F_{J}^{0}(V) \subset F_{J}^{1}(V) \subset \cdots \subset F_{J}^{s}(V)=V
$$

be a Jordan-Hölder filtration with $\sigma$-stable quotients $G^{i}(V)=F^{i}(V) / F^{i-1}(V)$. Now $W:=\bigoplus_{i} G^{i}(V) \in$ $X$ lies in the $\operatorname{GL}(Q, \alpha)_{\sigma}$ closure of $V$. In particular $\psi\left(\pi_{X}(W)\right)=\pi(V)$. This proves that $\psi$ is surjective.

Note that $W \in X$ is $G$-semi-stable if and only if all the summands in $\operatorname{Rep}\left(Q, \alpha_{i}\right)$ are $\sigma$-semi-stable. In particular, if $W \in X$ is $G$-semi-stable, then $W \sigma$-semi-stable, so $W$ is $\operatorname{GL}(Q, \alpha)_{\sigma}$-stable. This shows that $\psi^{-1}(0)=\{0\}$, so $\psi$ is a finite map.

For a general representation $V \in \operatorname{Rep}(Q, \alpha)$, the quotients of a Jordan-Hölder filtration corresponding to $\sigma$ are unique up to permutation. This shows that a generic fiber of $\psi$ consists of only one point. So $\psi$ is birational.

Because $\psi$ is birational and finite, and $\operatorname{Rep}(Q, \alpha) / / \operatorname{GL}(Q, \alpha)_{\sigma}$ is normal, $\psi$ must be an isomorphism. Now the graded coordinate ring of $\operatorname{Rep}(Q, \alpha) / / \operatorname{GL}(Q, \alpha)_{\sigma}$ is $\bigoplus_{m \geq 0} \operatorname{SI}(Q, \alpha)_{m \sigma}$ and $X / / G$ has graded coordinate ring

$$
\bigoplus_{m \geq 0} S^{c_{1}}\left(\mathrm{SI}\left(Q, \alpha_{1}\right)_{m \sigma}\right) \otimes S^{c_{2}}\left(\mathrm{SI}\left(Q, \alpha_{2}\right)_{m \sigma}\right) \otimes \cdots \otimes S^{c_{r}}\left(\mathrm{SI}\left(Q, \alpha_{r}\right)_{m \sigma}\right) .
$$

In particular we get

$$
\operatorname{SI}(Q, \alpha)_{m \sigma} \cong S^{c_{1}}\left(\operatorname{SI}\left(Q, \alpha_{1}\right)_{m \sigma}\right) \otimes S^{c_{2}}\left(\operatorname{SI}\left(Q, \alpha_{2}\right)_{m \sigma}\right) \otimes \cdots \otimes S^{c_{r}}\left(\operatorname{SI}\left(Q, \alpha_{r}\right)_{m \sigma}\right)
$$

for all $m \geq 0$.

Suppose that $K$ has arbitrary characteristic. We note that $\operatorname{dim} \operatorname{SI}(Q, \beta)_{\tau}$ is independent of the base field $K$ (see [16]) for any dimension vector $\beta$ and any weight $\tau$. The vector spaces $\operatorname{SI}(Q, \alpha)_{m \sigma}$ and

$$
S^{c_{1}}\left(\operatorname{SI}\left(Q, \alpha_{1}\right)_{m \sigma}\right) \otimes S^{c_{2}}\left(\operatorname{SI}\left(Q, \alpha_{2}\right)_{m \sigma}\right) \otimes \cdots \otimes S^{c_{r}}\left(\operatorname{SI}\left(Q, \alpha_{r}\right)_{m \sigma}\right)
$$

have the same dimension if $K$ has characteristic 0 . But then they have the same dimension even in the case where $K$ has positive characteristic. We have an isomorphism

$$
\mathrm{SI}(Q, \alpha)_{m \sigma} \cong S^{c_{1}}\left(\mathrm{SI}\left(Q, \alpha_{1}\right)_{m \sigma}\right) \otimes S^{c_{2}}\left(\mathrm{SI}\left(Q, \alpha_{2}\right)_{m \sigma}\right) \otimes \cdots \otimes S^{c_{r}}\left(\operatorname{SI}\left(Q, \alpha_{r}\right)_{m \sigma}\right)
$$

although this isomorphism may not be canonical. If we replace the symmetric powers $S^{c}$ by the divided powers $D^{c}$, then the argument in characteristic 0 still shows that we have a canonical injective map

$$
\operatorname{SI}(Q, \alpha)_{m \sigma} \hookrightarrow D^{c_{1}}\left(\operatorname{SI}\left(Q, \alpha_{1}\right)_{m \sigma}\right) \otimes D^{c_{2}}\left(\operatorname{SI}\left(Q, \alpha_{2}\right)_{m \sigma}\right) \otimes \cdots \otimes D^{c_{r}}\left(\operatorname{SI}\left(Q, \alpha_{r}\right)_{m \sigma}\right)
$$

Comparing dimensions shows that this map is an isomorphism.

\section{Schur SEQUENCES}

Proposition 3.18 and Corollary 3.19 motivates us to define Schur sequences. The notion of Schur sequences is closely related to the notion of exceptional sequences (see Section 2.7). A Schur sequence is similar to an exceptional sequence, but also imaginary Schur roots are allowed.

Definition 4.1. A sequence $\underline{\gamma}=\left(\gamma_{1}, \gamma_{2}, \ldots, \gamma_{s}\right)$ is called a Schur sequence if

(1) $\gamma_{i}$ is a Schur root for every $i$;

(2) $\gamma_{i} \Perp \gamma_{j}$ for all $i<j$.

Lemma 4.2. If $\operatorname{Rep}(Q, \alpha)$ has a dense $\operatorname{GL}(Q, \alpha)$-orbit or $\operatorname{Rep}(Q, \beta)$ has a dense $\operatorname{GL}(Q, \beta)$-orbit, then $\alpha \circ \beta \leq 1$. 
Proof. If $\operatorname{Rep}(Q, \beta)$ has a dense $\operatorname{GL}(Q, \beta)$-orbit, then there are no rational $\operatorname{GL}(Q, \beta)$-invariants in $K[\operatorname{Rep}(Q, \beta)]$. In particular, any quotient of two semi-invariants of the same weight must be constant. This shows that

$$
\alpha \circ \beta=\operatorname{dim} \operatorname{SI}(Q, \beta)_{\langle\alpha, \cdot\rangle} \leq 1 .
$$

If $\operatorname{Rep}(Q, \alpha)$ has a dense $\operatorname{GL}(Q, \alpha)$-orbit then the proof is similar.

Remark 4.3. An exceptional sequence $\underline{\varepsilon}=\left(\varepsilon_{1}, \varepsilon_{2}, \ldots, \varepsilon_{s}\right)$ is a Schur sequence. The space $\operatorname{Rep}\left(Q, \varepsilon_{j}\right)$ has a dense GL $\left(\varepsilon_{j}\right)$-orbit, so by Lemma 4.2

$$
\varepsilon_{i} \circ \varepsilon_{j}=\operatorname{dim} \operatorname{SI}\left(Q, \varepsilon_{j}\right)_{\left\langle\varepsilon_{i},\right\rangle} \leq 1 .
$$

This shows that $\varepsilon_{i} \Perp \varepsilon_{j}$ for all $i<j$.

Lemma 4.4. Suppose that $\alpha \perp \gamma$ and $\beta \perp \gamma$.

(a) If $(\alpha+\beta) \circ \gamma=1$, then $\alpha \circ \gamma=1$ and $\beta \circ \gamma=1$;

(b) if $\operatorname{ext}_{Q}(\alpha, \beta)=0, \alpha \circ \gamma=1$ and $\beta \circ \gamma=1$ then $(\alpha+\beta) \circ \gamma=1$.

Proof. (a) Since $\alpha \perp \gamma$ and $\beta \perp \gamma$ we have $\alpha \circ \gamma \geq 1$ and $\beta \circ \gamma \geq 1$.

Choose $f \in \operatorname{SI}(Q, \gamma)_{\langle\alpha, \cdot\rangle}$. Then we have

$$
f \operatorname{SI}(Q, \gamma)_{\langle\beta, \cdot\rangle} \subseteq \operatorname{SI}(Q, \gamma)_{\langle\alpha+\beta, \cdot\rangle} \cdot
$$

This shows that

$$
1 \leq \beta \circ \gamma=\operatorname{dim} \operatorname{SI}(Q, \gamma)_{\langle\beta, \cdot\rangle}=\operatorname{dim} f \operatorname{SI}(Q, \gamma)_{\langle\beta, \cdot\rangle} \leq \operatorname{dim} S I(Q, \gamma)_{\langle\alpha+\beta, \cdot\rangle}=(\alpha+\beta) \circ \gamma \leq 1 .
$$

Hence we get $\beta \circ \gamma=1$. Similarly we obtain $\alpha \circ \gamma=1$.

(b) Any $(\alpha+\beta)$-dimensional representation $V$ has an $\alpha$-dimensional subrepresentation $V^{\prime}$. If $V^{\prime \prime}=V / V^{\prime}$ then $c^{V}=c^{V^{\prime}} \cdot c^{V^{\prime \prime}}$ up to a scalar as functions on $\operatorname{Rep}(Q, \gamma)$ by Lemma 2.2. Since $\operatorname{SI}(Q, \gamma)_{\langle\alpha+\beta,\rangle}$ is spanned by semi-invariants of the form $c^{V}$ (see Theorem [2.3), we have

$$
\operatorname{SI}(Q, \gamma)_{\langle\alpha+\beta, \cdot\rangle}=\operatorname{SI}(Q, \gamma)_{\langle\alpha, \cdot\rangle} \operatorname{SI}(Q, \gamma)_{\langle\beta, \cdot\rangle} .
$$

It follows that

$$
1 \leq(\alpha+\beta) \circ \gamma \leq(\alpha \circ \gamma)(\beta \circ \gamma)=1 \cdot 1=1,
$$

so $(\alpha+\beta) \circ \gamma=1$.

Corollary 4.5. If $\gamma_{1}, \gamma_{2}, \ldots, \gamma_{s}$ is a Schur sequence, and $p \gamma_{i}+q \gamma_{i+1}$ is a Schur root, then

$$
\gamma_{1}, \gamma_{2}, \ldots, \gamma_{i-1}, p \gamma_{i}+q \gamma_{i+1}, \gamma_{i+2}, \ldots, \gamma_{s}
$$

is a Schur sequence.

Proof. This follows from Lemma 4.4(b) and Theorem 2.23

Remark 4.6. Suppose that $\alpha=\alpha_{1}^{\oplus c_{1}} \oplus \alpha_{2}^{\oplus c_{2}} \oplus \cdots \oplus \alpha_{s}^{\oplus c_{s}}$ is the canonical decomposition with all $\alpha_{i}$ distinct. By Theorem $2.13 \operatorname{ext}_{Q}\left(\alpha_{i}, \alpha_{j}\right)=0$ for all $i \neq j$. After reordering we may assume that $\operatorname{hom}_{Q}\left(\alpha_{i}, \alpha_{j}\right)=0$ for all $i<j$ by Lemma 2.15. So $\alpha_{i} \perp \alpha_{j}$ for all $i<j$. We claim that in fact $\alpha_{1}, \alpha_{2}, \ldots, \alpha_{s}$ is a Schur sequence. This follows from the algorithm in 14 for finding the canonical decomposition and Corollary 4.5

Remark 4.7. Suppose that $\alpha=c_{1} \cdot \alpha_{1} \dot{+} c_{2} \cdot \alpha_{2} \dot{+} \cdots \dot{+} c_{r} \cdot \alpha_{r}$ is a $(\sigma: \tau)$-stable decomposition. By Corollary 3.19 (c) we may assume that $\alpha_{i} \Perp \alpha_{j}$ for all $i<j$. By Corollary 3.19(a), $\alpha_{1}, \ldots, \alpha_{r}$ is a Schur sequence.

Definition 4.8. A Schur sequence $\underline{\gamma}=\left(\gamma_{1}, \gamma_{2}, \ldots, \gamma_{s}\right)$ is called a quiver Schur sequence if $\left\langle\gamma_{j}, \gamma_{i}\right\rangle \leq 0$ for all $i<j$. 
Remark 4.9. Suppose that $\alpha=c_{1} \cdot \alpha_{1} \dot{+} c_{2} \cdot \alpha_{2} \dot{+} \cdots \dot{+} c_{r} \cdot \alpha_{r}$ is a $\sigma$-stable decomposition. By Proposition 3.18 (d) we may assume that $\operatorname{ext}_{Q}\left(\alpha_{i}, \alpha_{j}\right)=0$ for all $i<j$. By Proposition 3.18 (a),(c), $\alpha_{1}, \alpha_{2}, \ldots, \alpha_{r}$ is a quiver Schur sequence.

Definition 4.10. Let $\underline{\gamma}=\left(\gamma_{1}, \ldots, \gamma_{r}\right), \underline{\beta}=\left(\beta_{1}, \ldots, \beta_{s}\right)$ be two sequences of dimension vectors. We say that $\beta$ is a refinement of $\gamma$ if there exists a sequence $0=b_{0}<b_{1}<\ldots<b_{r-1}<s=b_{r}$ such that for each $\bar{j}=1, \ldots, r$ the dimension vector $\gamma_{j}$ is a positive linear combination of $\beta_{b_{j-1}+1}, \ldots, \beta_{b_{j}}$.

Theorem 4.11 (Refinement Theorem). Let $\gamma=\left(\gamma_{1}, \ldots, \gamma_{r}\right)$ be a Schur sequence. Then there exists an exceptional sequence $\underline{\varepsilon}=\left(\varepsilon_{1}, \ldots, \varepsilon_{s}\right)$ such that $\underline{\varepsilon}$ is a refinement of $\underline{\gamma}$.

Proof. For a dimension vector $\alpha$ we define $\tau(\alpha)=\sum_{x \in Q_{0}} \alpha(x)$. We will prove the theorem by induction on the number of vertices $n$ in the quiver $Q$, and by induction on $\tau\left(\gamma_{1}\right)$. If $n=1$ there is nothing to prove. If $\tau\left(\gamma_{1}\right)=0$ then there is nothing to prove either since this is impossible.

Let us assume that in a Schur sequence the first dimension vector $\gamma_{1}$ is a real Schur root. Let $V$ be the unique indecomposable representation corresponding to the dense orbit in $\operatorname{Rep}\left(Q, \gamma_{1}\right)$. Then the dimension vectors $\gamma_{2}, \ldots, \gamma_{r}$ are Schur roots in the right orthogonal category $V^{\perp}$. By Theorem 2.28 the category $V^{\perp}$ is equivalent to the category of representations of a quiver $Q^{\prime}$ with no oriented cycles and $n-1$ vertices. Let $I: \mathbb{N}^{n-1} \rightarrow \mathbb{N}^{Q_{0}}$ as in Section 2.6. We can write $\gamma_{i}=I\left(\delta_{i}\right)$. Then $\delta_{2}, \ldots, \delta_{n}$ is a Schur sequence for $Q^{\prime}$. We can refine $\delta_{2}, \ldots, \delta_{n}$ to an exceptional sequence $\underline{\varepsilon}=\left(\varepsilon_{1}, \ldots, \varepsilon_{t}\right)$ by induction on $n$.

Then the sequence $\underline{\varepsilon}=\left(\gamma_{1}, I\left(\varepsilon_{2}\right), \ldots, I\left(\varepsilon_{n}\right)\right)$ is clearly an exceptional sequence for $Q$ which refines $\gamma$.

Next, assume that $\gamma_{1}$ is an imaginary root. Since $\gamma_{1} \circ \gamma_{j}=1$ for all $j \geq 2$, it follows by induction from Lemma 4.4 (b) that $\gamma_{1} \circ \delta=1$ where $\delta=\gamma_{2}+\cdots+\gamma_{r}$. Let $\gamma_{1}^{\perp}$ be the set of all dimension vectors $\alpha$ with $\gamma_{1} \perp \alpha$. By Theorem 2 from [13, $\mathbb{R}_{+} \gamma_{1}^{\perp}$ is a rational polyhedral cone in $\mathbb{R}^{n-1}$. Suppose that $\delta$ is in the interior of the cone. For each $\alpha \in \gamma_{1}^{\perp}$ there exists $\beta \in \gamma_{1}^{\perp}$ such that $\alpha+\beta=p \delta$ for some positive integer $p$. From Lemma 4.4 (a) follows that $\gamma_{1} \circ \alpha=1$. This shows that for all $\sigma \in \Gamma^{\star}, \operatorname{dim} \operatorname{SI}\left(Q, \gamma_{1}\right)_{\sigma} \leq 1$. Indeed, if $\operatorname{SI}\left(Q, \gamma_{1}\right)_{\sigma} \neq 0$ then $\sigma=-\langle\cdot, \alpha\rangle$ for some $\alpha \in \gamma_{1}^{\perp}$ and $\operatorname{dim} \operatorname{SI}\left(Q, \gamma_{1}\right)_{\sigma}=\gamma_{1} \circ \alpha=1$. This implies that all $\operatorname{GL}\left(\gamma_{1}\right)$-invariant rational functions on $\operatorname{Rep}\left(Q, \gamma_{1}\right)$ are constant. From this follows that $\operatorname{GL}\left(\gamma_{1}\right)$ has a dense orbit in $\operatorname{Rep}\left(Q, \gamma_{1}\right)$. Therefore, $\gamma_{1}$ must be a real Schur root. Contradiction, so it follows that $\delta$ is not in the interior of $\mathbb{R}_{+} \gamma_{1}^{\perp}$.

Let $\sigma=-\langle\cdot, \delta\rangle$ and let us study the $\sigma$-stable decomposition of $\gamma_{1}$. By Theorem 2.3 there exists a $\beta \hookrightarrow \gamma_{1}$ such that $\sigma(\beta)=0$. In particular, the $\sigma$-stable decomposition of $\gamma_{1}$ is nontrivial. Suppose that

$$
\gamma_{1}=c_{1} \cdot \beta_{1} \dot{+} c_{2} \cdot \beta_{2} \dot{+} \cdots \dot{+} c_{l} \cdot \beta_{l}
$$

is the $\sigma$-stable decomposition of $\gamma_{1}$. We may assume that $\beta_{i} \Perp \beta_{j}$ for $i<j$. From $\gamma_{1} \Perp \delta=1$ and Lemma 4.4 follows that $\beta_{i} \Perp \gamma_{j}$ for all $j \geq 2$ and all $i$. Therefore

$$
\underline{\gamma^{\prime}}=\left(\beta_{1}, \beta_{2}, \ldots, \beta_{l}, \gamma_{2}, \ldots, \gamma_{r}\right)
$$

is a Schur sequence using Lemma 4.4 (a). Notice that $\beta_{1}$ is smaller than $\gamma_{1}$. Now $\tau\left(\beta_{1}\right)<\tau\left(\gamma_{1}\right)$ so by induction there exists an exceptional sequence which is a refinement of $\underline{\gamma}^{\prime}$.

Corollary 4.12. Let $\underline{\gamma}=\left(\gamma_{1}, \ldots, \gamma_{r}\right)$ be a Schur sequence. Then the vectors $\gamma_{1}, \ldots, \gamma_{r}$ are linearly independent.

Proof. First assume that $\underline{\gamma}$ is an exceptional sequence. We have

$$
\left\langle\gamma_{i}, \gamma_{j}\right\rangle= \begin{cases}1 & \text { if } i=j \\ 0 & \text { if } i<j\end{cases}
$$


The matrix

is invertible, so $\gamma_{1}, \ldots, \gamma_{r}$ are linearly independent.

$$
\left(\left\langle\gamma_{i}, \gamma_{j}\right\rangle\right)_{i, j=1}^{n}
$$

If $\gamma$ is not an exceptional sequence then it has a refinement which is an exceptional sequence. So again, it follows that $\gamma_{1}, \ldots, \gamma_{r}$ are linearly independent.

\section{The Faces of The CONE $\mathbb{R}_{+} \Sigma(Q, \alpha)$}

As before, $\Sigma(Q, \alpha)$ denotes the set of all weights $\sigma \in \Gamma^{\star}$ such that $\alpha$ is $\sigma$-semi-stable. If $\alpha$ is a sincere dimension vector, (i.e., $\alpha(x)>0$ for all $x \in Q_{0}$,) then there is a bijection between $\alpha^{\perp}=\{\beta \in$ $\left.\mathbb{N}^{Q_{0}} \mid \alpha \perp \beta\right\}$ and $\Sigma(Q, \alpha)$ by

$$
\beta \in \alpha^{\perp} \leftrightarrow \sigma:=-\langle\cdot, \beta\rangle \in \Sigma(Q, \alpha) .
$$

Similarly there is also a bijection between $\Sigma(Q, \alpha)$ and ${ }^{\perp} \alpha$.

Let $\mathbb{R}_{+}$be the set of nonnegative real numbers. We consider the cone $\mathbb{R}_{+} \Sigma(Q, \alpha) \subseteq \mathbb{R}_{+} \otimes_{\mathbb{Z}} \Gamma^{\star} \cong$ $\left(\mathbb{R}^{n}\right)^{\star}$. This cone is of particular interest. For example, in a special case, this cone is equal to the Klyachko cone. In this section, we will unravel the geometry of this cone.

The refinement Theorem (Theorem 4.11) allows us to obtain a beautiful description of the faces of $\mathbb{R}_{+} \Sigma(Q, \alpha)$. Let us denote by $\mathcal{W}_{r}(Q, \alpha)$ the set of all sets $\left\{\gamma_{1}, \ldots, \gamma_{r}\right\}$ such that $\underline{\gamma}=\left(\gamma_{1}, \ldots, \gamma_{r}\right)$ is a quiver Schur sequence of length $r$ such that $\alpha=\sum_{i=1}^{r} a_{i} \gamma_{i}$ with

(1) $a_{i}$ a positive integer for all $i$;

(2) if $\gamma_{i}$ is imaginary and not isotropic, then $a_{i}=1$.

Let $\mathcal{F}_{r}(Q, \alpha)$ be the set of faces of dimension $n-r$ of $\mathbb{R}_{+} \Sigma(Q, \alpha)$.

Theorem 5.1. Let $Q$ be a quiver without oriented cycles and let $\alpha$ be a dimension vector. For each $r, 1 \leq r \leq n-1$ there is a natural bijection

$$
\psi(r): \mathcal{W}_{r}(Q, \alpha) \rightarrow \mathcal{F}_{r}(Q, \alpha)
$$

which sends the quiver Schur sequence $\underline{\gamma}=\left(\gamma_{1}, \ldots, \gamma_{r}\right)$ to the face

$$
\mathbb{R}_{+} \Sigma\left(Q, \gamma_{1}\right) \cap \ldots \cap \mathbb{R}_{+} \Sigma\left(Q, \gamma_{r}\right)=\mathbb{R}_{+} \Sigma(Q, \alpha) \cap\left\{\sigma \in\left(\mathbb{R}^{n}\right)^{\star} \mid \sigma\left(\gamma_{1}\right)=\cdots=\sigma\left(\gamma_{r}\right)=0\right\} .
$$

The inverse bijection is obtained as follows. For a given face $F$ we take a weight $\sigma \in \Gamma^{\star}$ in the relative interior of $F$ and associate to it the quiver Schur sequence coming from the $\sigma$-stable decomposition of $\alpha$.

Proof. Suppose that $\underline{\gamma}=\left(\gamma_{1}, \ldots, \gamma_{r}\right) \in \mathcal{W}_{r}(Q, \alpha)$. Let us first prove that

$$
\mathbb{R}_{+} \Sigma\left(Q, \gamma_{1}\right) \cap \ldots \cap \mathbb{R}_{+} \Sigma\left(Q, \gamma_{r}\right)
$$

is a face of codimension $r$ in the space of dimension vectors. Clearly

$$
\Sigma\left(Q, \gamma_{1}\right) \cap \ldots \cap \Sigma\left(Q, \gamma_{r}\right) \subseteq \Sigma(Q, \alpha) \cap\left\{\sigma \in \Gamma^{\star} \mid \sigma\left(\gamma_{1}\right)=\cdots=\sigma\left(\gamma_{r}\right)=0\right\} .
$$

The converse inequality

$$
\Sigma\left(Q, \gamma_{1}\right) \cap \ldots \cap \Sigma\left(Q, \gamma_{r}\right) \supseteq \Sigma(Q, \alpha) \cap\left\{\sigma \in \Gamma^{\star} \mid \sigma\left(\gamma_{1}\right)=\cdots=\sigma\left(\gamma_{r}\right)=0\right\} .
$$

also holds: If $\alpha$ is $\sigma$-semi-stable, and $\sigma\left(\gamma_{1}\right)=\cdots=\sigma\left(\gamma_{r}\right)=0$ then $\gamma_{1}, \ldots, \gamma_{r}$ are $\sigma$-semi-stable. We conclude that

$$
\Sigma\left(Q, \gamma_{1}\right) \cap \ldots \cap \Sigma\left(Q, \gamma_{r}\right)=\Sigma(Q, \alpha) \cap\left\{\sigma \in \Gamma^{\star} \mid \sigma\left(\gamma_{1}\right)=\cdots=\sigma\left(\gamma_{r}\right)=0\right\} .
$$

Using the saturation property, we get

$$
\mathbb{R}_{+} \Sigma\left(Q, \gamma_{1}\right) \cap \ldots \cap \mathbb{R}_{+} \Sigma\left(Q, \gamma_{r}\right)=\mathbb{R}_{+} \Sigma(Q, \alpha) \cap\left\{\sigma \in\left(\mathbb{R}^{n}\right)^{\star} \mid \sigma\left(\gamma_{1}\right)=\cdots=\sigma\left(\gamma_{r}\right)=0\right\}
$$


By the refinement Theorem (Theorem 4.11) there exists an exceptional sequence $\underline{\varepsilon}=\left(\varepsilon_{1}, \ldots, \varepsilon_{s}\right)$ which is a refinement of $\gamma$, i.e., there exists a sequence $0=b_{0}<b_{1}<\ldots<b_{r-1}<n=b_{r}$ such that for each $j=1, \ldots, r$ the dimension vector $\gamma_{j}$ is a positive linear combination of $\varepsilon_{b_{j-1}+1}, \ldots, \varepsilon_{b_{j}}$.

We proceed by induction on $r$. Suppose that $r=1$. Then $\alpha$ is a integer multiple of $\gamma_{1}$. We have $\Sigma(Q, \alpha)=\Sigma\left(Q, \gamma_{1}\right)$. The cone $\mathbb{R}_{+} \Sigma(Q, \alpha)=\mathbb{R}_{+} \Sigma\left(Q, \gamma_{1}\right)$ is given by one equality, $\sigma\left(\gamma_{1}\right)=0$ and many inequalities of the form $\sigma(\beta) \leq 0$ for all $\beta \hookrightarrow \gamma_{1}$. There exists a weight $\tau$ such that $\gamma_{1}$ is $\tau$-stable. This means that $\tau(\beta)<0$ for all $\beta \hookrightarrow \gamma_{1}$ and $\beta \neq 0, \gamma_{1}$. If we view $\sigma, \mathbb{R}_{+} \Sigma(Q, \alpha)$ inside the space

$$
\left\{\sigma \in\left(\mathbb{R}^{n}\right)^{\star} \mid \sigma\left(\gamma_{1}\right)=0\right\} \cong \mathbb{R}^{n-1}
$$

then $\mathbb{R}_{+} \Sigma(Q, \alpha)$ contains an open neighborhood of $\tau$. This shows that $\mathbb{R}_{+} \Sigma(Q, \alpha)=\mathbb{R}_{+} \Sigma\left(Q, \gamma_{1}\right)$ is a cone of dimension $n-1$. In particular $\mathbb{R}_{+} \Sigma(Q, \alpha)$ is a face of dimension $n-1$.

Suppose that $r>1$. Let $V_{i}$ be the unique representation corresponding to the dense orbit in $\operatorname{Rep}\left(Q, \varepsilon_{i}\right)$, for $i=1,2, \ldots, b_{1}$. The representations $V_{1}, \ldots, V_{b_{1}}$ generate the full subcategory $\mathcal{C}\left(V_{1}, \ldots, V_{b_{1}}\right)$ of $\operatorname{Rep}_{K}(Q)$ which is closed under extensions, direct sums, and taking kernels and cokernels. This category is equivalent to the category of representations of some quiver $Q^{\prime}$ with $b_{1}$ vertices and without oriented cycles. (See Lemma 2.35 and Theorem 2.28). The right orthogonal category

$$
\left(V_{1}, \ldots, V_{b_{1}}\right)^{\perp}=V_{1}^{\perp} \cap \cdots \cap V_{b_{1}}^{\perp}
$$

is the category of representations of a quiver $Q^{\prime \prime}$ with $n-b_{1}$ vertices and without oriented cycles (Theorem 2.28). Define $I^{\prime}: \mathbb{N}^{Q_{0}^{\prime}} \rightarrow \mathbb{N}^{Q_{0}}$ and $I^{\prime \prime}: \mathbb{N}^{Q_{0}^{\prime \prime}} \rightarrow \mathbb{N}^{Q_{0}}$ as in Theorem 2.39.

By the induction hypothesis, we can find linearly independent

$$
\tau_{1}^{\prime}, \ldots, \tau_{b_{1}-1}^{\prime} \in \Sigma\left(Q^{\prime},\left(I^{\prime}\right)^{-1}\left(\gamma_{1}\right)\right) .
$$

and linearly independent

$$
\tau_{1}^{\prime \prime}, \ldots, \tau_{n-b_{1}-r+1}^{\prime \prime} \in \Sigma\left(Q^{\prime \prime},\left(I^{\prime \prime}\right)^{-1}\left(\gamma_{2}\right)\right) \cap \cdots \cap \Sigma\left(Q^{\prime \prime},\left(I^{\prime \prime}\right)^{-1}\left(\gamma_{r}\right)\right) .
$$

Define $\sigma_{1}^{\prime}, \ldots, \sigma_{b_{1}-1}^{\prime} \in \Gamma^{\star}$ such that $\sigma_{i}^{\prime} \circ I^{\prime}=\tau_{i}^{\prime}$ and $\sigma_{i}^{\prime} \circ I^{\prime \prime}=0$ for $i=1,2, \ldots, b_{1}-1$. Define $\sigma_{1}^{\prime \prime}, \ldots, \sigma_{n-b_{1}-r-1}^{\prime \prime} \in \Gamma^{\star}$ by $\sigma_{i}^{\prime \prime} \circ I^{\prime \prime}=\tau_{i}^{\prime \prime}$ and $\sigma_{i}^{\prime \prime} \circ I=0$ for $i=1,2, \ldots, n-b_{1}-r-1$. Then

$$
\sigma_{1}^{\prime}, \ldots, \sigma_{b_{1}-1}^{\prime}, \sigma_{1}^{\prime \prime}, \ldots, \sigma_{n-b_{1}-r+1}^{\prime \prime} \in \Sigma\left(Q, \gamma_{1}\right) \cap \cdots \cap \mathbb{R}_{+} \Sigma\left(Q, \gamma_{r}\right)
$$

are $n-r$ linearly independent weights. This shows that the face

$$
\mathbb{R}_{+} \Sigma\left(Q, \gamma_{1}\right) \cap \cdots \cap \mathbb{R}_{+} \Sigma\left(Q, \gamma_{r}\right)
$$

has dimension $n-r$.

We have proved that the map $\psi(r)$ defined above is well defined. Let us show that the inverse map is well defined and that it is indeed an inverse. Let $F$ be a face of dimension $n-r$ of $\mathbb{R}_{+} \Sigma(Q, \alpha)$. Take a weight $\sigma \in \Gamma^{\star}$ in the relative interior of $F$. Let

$$
\alpha=c_{1} \cdot \delta_{1} \dot{+} c_{2} \cdot \delta_{2} \dot{+} \cdot \dot{+} c_{l} \cdot \delta_{l}
$$

be the $\sigma$-stable decomposition of $\alpha$. Define $F^{\prime}=\mathbb{R}_{+} \Sigma\left(Q, \delta_{1}\right) \cap \cdots \cap \Sigma\left(Q, \delta_{l}\right)$. Since $\sigma \in F^{\prime}$ and $\sigma$ is in the relative interior of $F$, we have $F \subseteq F^{\prime}$.

Suppose that $\gamma \hookrightarrow \alpha$ and $\sigma(\gamma)=0$. Then $\gamma$ is a linear combination of the $\delta_{i}$ 's by the definition of $\sigma$-stable decomposition. But the description of $\Sigma(Q, \alpha)$ given in Theorem 2.9 implies that

$$
\begin{gathered}
F^{\prime}=\mathbb{R}_{+} \Sigma(Q, \alpha) \cap\left\{\sigma \in\left(\mathbb{R}^{n}\right)^{\star} \mid \sigma\left(\delta_{1}\right)=\cdots=\sigma\left(\delta_{l}\right)=0\right\} \subseteq \\
\subseteq F=\mathbb{R}_{+} \Sigma(Q, \alpha) \cap \bigcap_{\gamma, \gamma \hookrightarrow \alpha,\langle\gamma, \beta\rangle=0}\left\{\sigma \in\left(\mathbb{R}^{n}\right)^{\star} \mid \sigma(\gamma)=0\right\}
\end{gathered}
$$

so we have $F^{\prime} \subseteq F$. This concludes the proof of the Theorem. 
Let us state the meaning of Theorem 5.1 in two extreme cases: for the walls of maximal dimension of $\Sigma(Q, \alpha)$ and for extremal rays.

Corollary 5.2. Let $Q$ be a quiver without oriented cycles and let $\alpha$ be a Schur root. Then the walls of $\Sigma(Q, \alpha)$ (i.e., faces of dimension $n-2)$ are in one to one correspondence with the ways of writing

$$
\alpha=c_{1} \gamma_{1}+c_{2} \gamma_{2}
$$

where $\left(\gamma_{1}, \gamma_{2}\right)$ is a quiver Schur sequence, $c_{1}, c_{2}$ positive integers with $c_{i}=1$ whenever $\gamma_{i}$ is imaginary and non-isotropic.

Let us consider an extremal ray $\sigma$ in $\Sigma(Q, \alpha)$. It corresponds to the linear combination

$$
\alpha=c_{1} \gamma_{1}+\ldots+c_{n-1} \gamma_{n-1}
$$

where $\left(\gamma_{1}, \ldots, \gamma_{n-1}\right)$ is a quiver Schur sequence. Theorem 4.11 implies that at least $n-2$ of the roots $\gamma_{1}, \ldots, \gamma_{n-1}$ are real Schur roots. Consider the subring

$$
\mathrm{SI}(Q, \alpha, \sigma)=\bigoplus_{m \geq 0} \mathrm{SI}(Q, \alpha)_{m \sigma}
$$

By peeling off real roots from the left and from the right we can reduce the calculation of this ring to the ring of semi-invariants for a quiver $\theta(\ell)$ where $\theta(\ell)$ is the Kronecker quiver $\theta(\ell)$ with two vertices and $\ell$ equioriented arrows.

Corollary 5.3. Let $Q$ be a quiver with no oriented cycles and let $\alpha$ be a Schur root. Suppose that $\sigma \in \Sigma(Q, \alpha)$ is indivisible and spans an extremal ray. Then there exists a positive integer $n$ and a Schur root $\beta$ for the quiver $\theta(\ell)$ such that

$$
\operatorname{SI}(Q, \alpha, \sigma) \cong \operatorname{SI}(\theta(\ell), \beta)
$$

Example 5.4. Let $Q$ be the quiver

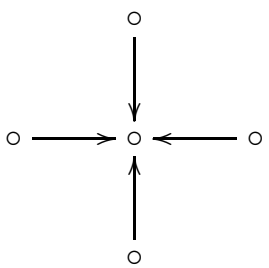

and let $\alpha$ be the dimension vector

$$
\begin{array}{rrr} 
& 1 & \\
1 & 2 & 1 \\
& 1
\end{array}
$$

There are 8 walls of $\Sigma(Q, \alpha)$. They are given by the Schur sequences

$$
\begin{array}{rrrrrrr} 
& 0 & & & 1 & & \\
1 & 2 & 1, & 0 & 0 & 0 & \text { (4 by symmetry) } \\
& 1 & & & 0 & & \\
& & & & & & \\
& 1 & & & 0 & & \\
0 & 1 & 0, & 1 & 1 & 1 &
\end{array}
$$

There are 12 two-dimensional faces of the cone given by the sequences

$$
\begin{array}{lllllllllll}
1 & & & 0 & & & 0 & & \\
1 & 0 & 1 & 1 & 0 & 0 & 0 & 1 & \\
0 & & & 1
\end{array}
$$


There are 6 extremal rays, given by the Schur sequences

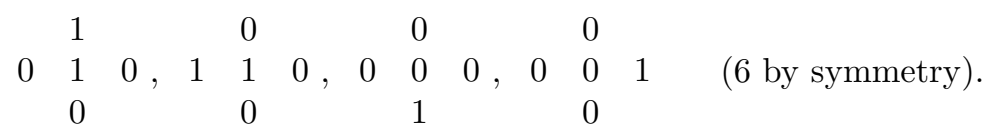

The set $\Sigma(Q, \alpha)$ is a cone over a regular octahedron.

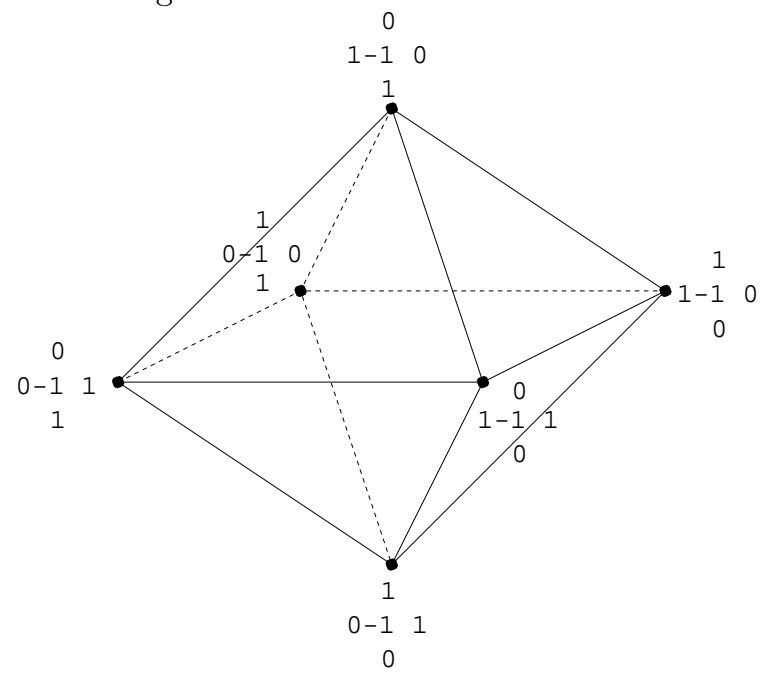

Example 5.5. Let $Q$ be the quiver

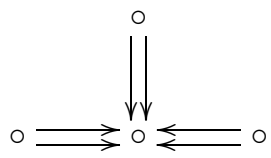

and let $\alpha$ be the dimension vector

$\begin{array}{lll} & 1 & \\ 1 & 3 & 1\end{array}$.

Now $\Sigma(Q, \alpha)$ has 6 walls corresponding to the Schur sequences

$$
\begin{array}{llllllll} 
& 0 & & & 1 & & \\
1 & 3 & 1 & 0 & 0 & 0 & (3 \text { by symmetry }) \\
& & & & & & \\
& 1 & & & 0 & & \\
0 & 2 & 0 & 1 & 1 & 1 & \text { (3 by symmetry) }
\end{array}
$$

The are also 6 extremal rays which correspond to the Schur sequences

$$
\left.\begin{array}{lllllllllll} 
& 1 & & & 0 & & & 0 & & & \\
0 & 2 & 0 & 1 & 1 & 0 & 0 & 0 & 0 & 1
\end{array} \text { by symmetry }\right) .
$$


The cone $\Sigma(Q, \alpha)$ is a cone over a hexagon.

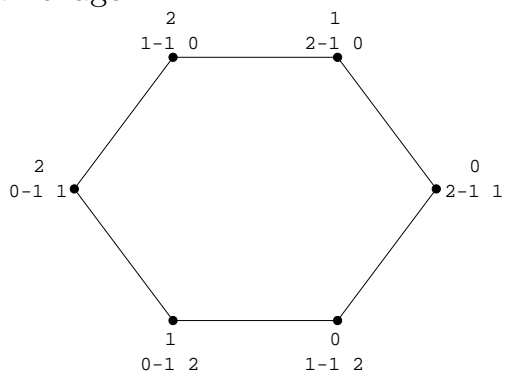

\section{More on the $\sigma$-Stable DeCompositions}

6.1. The set of $\sigma$-stable dimension vectors. In the previous section we studied, how the $\sigma$-stable decomposition of $\alpha$ varies, when $\sigma$ varies and $\alpha$ is fixed. This led to the description of the faces of $\Sigma(Q, \alpha)$. In this section, we will study how the $\sigma$-stable decomposition looks like for a fixed weight $\sigma$. Let us define $\bar{\Sigma}(Q, \sigma)$ as the set of all $\sigma$-semi-stable dimension vectors. Notice that

$$
\alpha \in \bar{\Sigma}(Q, \sigma) \quad \Leftrightarrow \quad \sigma \in \Sigma(Q, \alpha) .
$$

Lemma 6.1. Suppose that $\alpha \in \bar{\Sigma}(Q, \sigma)$. There exists a sequence $\underline{\delta}=\delta_{1}, \ldots, \delta_{s}$ of dimension vectors such that

(1) $\alpha=\sum_{i=1}^{s} a_{i} \delta_{i}$ for some positive rational numbers $a_{1}, \ldots, a_{s}$;

(2) $\delta_{1}, \ldots, \delta_{s}$ are linearly independent dimension vectors;

(3) each $\delta_{i}$ generates an extremal ray, i.e., there exists an extremal ray $\mathcal{R}_{i}$ of $\mathbb{R}_{+} \bar{\Sigma}(Q, \sigma)$ such that $\mathcal{R}_{i} \cap \bar{\Sigma}(Q, \sigma)=\mathbb{N} \delta_{i}$.

Proof. This is trivial.

Lemma 6.2. Suppose that $\alpha, \beta, \delta_{1}, \ldots, \delta_{s}$ are $\sigma$-stable, and $\beta \hookrightarrow \alpha$.

(a) If $\alpha$ is a nonnegative integral combination of $\delta_{1}, \ldots, \delta_{s}$ then so is $\beta$.

(b) If $\alpha$ is a nonnegative rational combination of $\delta_{1}, \ldots, \delta_{s}$ then so is $\beta$.

Proof. Suppose that $\alpha=\sum_{i=1}^{s} a_{i} \delta_{i}$ for some integers $a_{1}, \ldots, a_{s}$. Note that $\delta_{i}$ is $\sigma$-stable for all $i$ because $\delta_{i}$ is not the sum of two smaller $\sigma$-semi-stable dimension vectors. Let $V_{i}$ be $\sigma$-stable of dimension $\delta_{i}$ for all $i$. Consider the representation

$$
V=V_{1}^{a_{1}} \oplus V_{2}^{a_{2}} \oplus \cdots \oplus V_{s}^{a_{s}} .
$$

Now $V$ has a semi-stable subrepresentation $W$ of dimension $\beta$ by Lemma 3.7. In a Jordan-Hölder filtration of $W$, only $V_{1}, \ldots, V_{s}$ will appear, so $\beta$ must be a nonnegative integral combination of $\delta_{1}, \ldots, \delta_{s}$.

The second statement follows from the fact that for each positive integer $m$ we have (see Theorem 2.7)

$$
\beta \hookrightarrow \alpha \quad \Rightarrow \quad m \beta \hookrightarrow m \alpha .
$$

Definition 6.3. For a sequence of roots $\underline{\alpha}=\left(\alpha_{1}, \ldots, \alpha_{s}\right)$ (all $\alpha_{i}$ distinct) with $\left\langle\alpha_{i}, \alpha_{j}\right\rangle \leq 0$ for $i \neq j$, we define a quiver $Q(\underline{\alpha})$ as follows. The set of vertices of $Q(\underline{\alpha})_{0}$ is equal to $\{1,2, \ldots, s\}$. For $i \neq j$ there are $-\left\langle\alpha_{i}, \alpha_{j}\right\rangle$ arrows from $i$ to $j$. There are $1-\left\langle\alpha_{i}, \alpha_{i}\right\rangle$ arrows (loops) from $i$ to $i$.

Theorem 6.4. Under the assumptions of Lemma 6.1 above, $\alpha$ is $\sigma$-stable if and only if 
(1) either $\alpha=\delta_{i}$ and $\delta_{i}$ is a real Schur root for some $i$,

(2) or $\left\langle\delta_{i}, \alpha\right\rangle \leq 0$ and $\left\langle\alpha, \delta_{i}\right\rangle \leq 0$ for all $i, Q(\underline{\delta})$ is path connected and $\alpha$ is indivisible if $\alpha$ is isotropic.

Proof. First we prove that the conditions are necessary. Assume that $\alpha$ is $\sigma$-stable. For $i \neq j$ we have $\operatorname{hom}_{Q}\left(\delta_{j}, \delta_{i}\right)=0$ because $\delta_{i}, \delta_{j}$ are $\sigma$-stable (see Lemma 3.10). Suppose that $\left\langle\alpha, \delta_{i}\right\rangle>0$. This is only possible when $\delta_{i}$ is a real Schur root because $\left\langle\delta_{j}, \delta_{i}\right\rangle \leq 0$ for all $j \neq i$. In that case we have $\operatorname{hom}_{Q}\left(\alpha, \delta_{i}\right) \neq 0$, so $\alpha=\delta_{i}$ because $\alpha$ and $\delta_{i}$ are $\sigma$-stable (see Lemma 3.10). Similarly, if $\left\langle\delta_{i}, \alpha\right\rangle>0$ then we have $\alpha=\delta_{i}$.

Consider the quiver $Q(\underline{\delta})$. Let $S_{1}$ be the set of all $k$ such that there is a path from 1 to $k$ and let $S_{2}=S \backslash S_{1}$. Define

$$
\alpha_{1}=\sum_{i \in S_{1}} a_{i} \delta_{i}, \quad \alpha_{2}=\sum_{i \in S_{2}} a_{i} \delta_{i}
$$

There are no arrows from $S_{1}$ to $S_{2}$. This shows that $\left\langle\alpha_{1}, \alpha_{2}\right\rangle=0$. Choose an integer $m$ such that $m a_{i}$ is a positive integer for all $i$. We have $m \alpha_{1} \hookrightarrow m \alpha$. Since $\alpha$ is $\sigma$-stable, the $\sigma$-stable decomposition of $m \alpha$ is either $m \alpha$ or $\alpha \dot{+} \cdots \dot{+} \alpha$ by Proposition 3.16. Because $m \alpha_{1}$ is $\sigma$-semi-stable, $m \alpha_{1}$ must be proportional to $\alpha$. This can only happen if $S_{2}=\emptyset$. We have shown that the quiver $Q(\underline{\delta})$ is path connected.

If $\alpha$ is isotropic, then $\alpha$ must be indivisible because of Proposition 3.17

Clearly, if condition (1) is satisfied, then $\alpha$ is $\sigma$-stable. Suppose that (2) is satisfied. Suppose that $\beta \hookrightarrow \alpha, \beta$ is $\sigma$-stable and $\beta \neq 0, \alpha$. We will derive a contradiction. By Lemma $6.2 \beta=\sum_{i=1}^{s} b_{i} \delta_{i}$ such that the $b_{i}$ 's are nonnegative rational numbers. Define

$$
T_{1}=\operatorname{Supp}(\beta):=\left\{i \mid 1 \leq i \leq s, b_{i} \neq 0\right\}
$$

Let

$$
T_{2}=\{1,2, \ldots, s\} \backslash T_{1}=\left\{i \mid 1 \leq i \leq s, b_{i}=0\right\} .
$$

Assume that $T_{2} \neq \emptyset$. Define

$$
\alpha_{1}=\sum_{i \in T_{1}} a_{i} \delta_{i}, \quad \alpha_{2}=\sum_{i \in T_{2}} a_{i} \delta_{i} .
$$

Now $\alpha=\alpha_{1}+\alpha_{2}$. Because $\left\langle\delta_{i}, \delta_{j}\right\rangle \leq 0$ for all $i \neq j$, and because there must be at least one arrow from $T_{1}$ to $T_{2}$, we get $\left\langle\beta, \alpha_{2}\right\rangle<0$. Since $\operatorname{ext}_{Q}(\beta, \alpha-\beta)=0$, we get $\langle\beta, \alpha-\beta\rangle \geq 0$, so $\langle\beta, \gamma\rangle>0$ with

$$
\gamma=(\alpha-\beta)-\alpha_{2}=\alpha_{1}-\beta=\sum_{\alpha_{i} \in T_{1}}\left(a_{i}-b_{i}\right) \delta_{i} .
$$

If

$$
\gamma=\gamma_{1} \dot{+} \gamma_{2} \dot{+} \cdots \dot{+} \gamma_{r}
$$

is the $\sigma$-stable decomposition of $\gamma$, then $\left\langle\beta, \gamma_{j}\right\rangle>0$ for some $j$. We get that $\beta=\gamma_{j}$ by Lemma 3.10. But then

Contradiction.

$$
\left\langle\beta, \gamma_{j}\right\rangle=\langle\beta, \beta\rangle=\langle\beta, \alpha\rangle-\langle\beta, \alpha-\beta\rangle \leq 0
$$

This shows that $T_{2}=\emptyset$ and $T_{1}=\operatorname{Supp}(\beta)=\{1,2, \ldots, s\}$. Let $\gamma=\alpha-\beta$. We have $\gamma \neq 0, \alpha$. We can find a $\sigma$-stable dimension vector $\gamma^{\prime}$ such that $\gamma \rightarrow \gamma^{\prime}$ and therefore $\alpha \rightarrow \gamma^{\prime}$. By a similar argument as before we obtain $\operatorname{Supp}\left(\gamma^{\prime}\right)=\operatorname{Supp}(\gamma)=\{1,2, \ldots, s\}$. Write $\gamma=\sum_{i=1}^{s} c_{i} \delta_{i}$ with $c_{i}$ a positive rational number for all $i$. We have

$$
0=\langle\beta, \gamma\rangle=\sum_{i=1}^{s} c_{i}\left\langle\beta, \delta_{i}\right\rangle
$$


Since $\beta$ and $\delta_{i}$ are $\sigma$-stable, and $\beta \neq \delta_{i}$ we get that $\operatorname{hom}_{Q}\left(\beta, \delta_{i}\right)=0$ by Lemma 3.10. In particular, $\left\langle\beta, \delta_{i}\right\rangle \leq 0$ for all $i$. Combined with 19 we conclude that $\left\langle\beta, \delta_{i}\right\rangle=0$ for all $i$.

Let $B=\max \left\{b_{1}, \ldots, b_{s}\right\}$. Let $S_{1}=\left\{i \mid b_{i}=B\right\}$ and let $S_{2}=\{1,2, \ldots, s\} \backslash S_{1}$. Suppose $S_{2} \neq \emptyset$. There must be an arrow from $S_{1}$ to $S_{2}$, say $j \rightarrow k$ with $j \in S_{1}$ and $k \in S_{2}$. Then

$$
0=\left\langle\beta, \delta_{k}\right\rangle \leq b_{j}\left\langle\delta_{j}, \delta_{k}\right\rangle+b_{k}\left\langle\delta_{k}, \delta_{k}\right\rangle
$$

We know that $b_{j}>b_{k},\left\langle\delta_{j}, \delta_{k}\right\rangle \leq-1$, and $\left\langle\delta_{k}, \delta_{k}\right\rangle \leq 1$. This leads to a contradiction.

So $S_{2}=\emptyset$ and $b_{i}=B$ for all $i$. From $\left\langle\beta, \delta_{i}\right\rangle=0$ follows that for every $i$ there is exactly one arrow with tail $i$. Since $Q(\underline{\delta})$ is path connected, the quiver $Q(\underline{\delta})$ has to be a cycle. Now it easily follows from $\left\langle\alpha, \delta_{i}\right\rangle \leq 0$ that $\alpha$ must be proportional to $\beta$ and $\langle\alpha, \alpha\rangle=0$. Since $\alpha$ is indivisible in that case, $\alpha=\beta$.

Remark 6.5. The previous theorem also provides us an inductive way of finding the $\sigma$-stable decomposition, if $\alpha$ is $\sigma$-semi-stable, but not $\sigma$-stable. There are two cases.

In the first case $\left\langle\delta_{i}, \alpha\right\rangle>0$ or $\left\langle\alpha, \delta_{i}\right\rangle>0$ for some extremal dimension vector $\delta_{i} \in \bar{\Sigma}(Q, \sigma)$. If we know the $\sigma$-stable decomposition of the smaller dimension vector $\alpha-\delta_{i}$, we know the $\sigma$-stable decomposition of $\alpha$.

In the second case $Q(\underline{\delta})$ is not path-connected, say there is no path from $i$ to $j$. Let $S_{1}$ be the set of all $k$ such that there is a path from $i$ to $k$ and let $S_{2}$ be the complement. There are no arrows from $S_{1}$ to $S_{2}$. If we define

$$
\alpha_{1}=\sum_{i \in S_{1}} a_{i} \delta_{i}, \quad \alpha_{2}=\sum_{i \in S_{2}} a_{i} \delta_{i}
$$

For some $m, m \alpha_{1}, m \alpha_{2}$ are dimension vectors, and $m \alpha_{1} \hookrightarrow m \alpha$. If we know the $\sigma$-stable decomposition of $m \alpha_{1}$ and $m \alpha_{2}$ then we know the $\sigma$-stable decomposition of $m \alpha$ and $\alpha$. Now $m \alpha_{1}$ and $m \alpha_{2}$ have smaller support than $\alpha$.

Example 6.6. Let $Q$ be the quiver

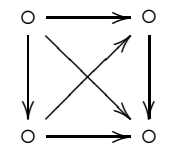

and let $\sigma$ be the weight

$$
\begin{array}{ll}
1 & -1 \\
1 & -1
\end{array}
$$

The extremal rays of the cone $\bar{\Sigma}(Q, \sigma)$ are given by the dimension vectors

$$
\delta_{1}=\begin{array}{ll}
1 & 1 \\
0 & 0
\end{array}, \quad \delta_{2}=\begin{array}{lllll}
1 & 0 \\
0 & 1
\end{array}, \quad \delta_{3}=\begin{array}{lll}
0 & 0 \\
1 & 1
\end{array} \quad \delta_{4}=\begin{array}{ll}
0 & 1 \\
1 & 0
\end{array} .
$$

The quiver $Q(\underline{\delta})$ is

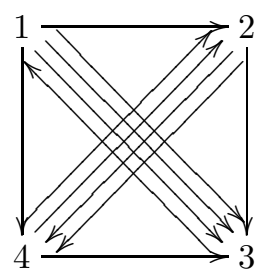

Any $\sigma$-stable dimension vector is a nonnegative rational combination of $\delta_{1}, \delta_{2}, \delta_{4}$ or a nonnegative rational combination of $\delta_{2}, \delta_{3}, \delta_{4}$. Suppose $\alpha$ is $\sigma$-stable and not equal to $\delta_{1}, \delta_{2}, \delta_{3}, \delta_{4}$. If $\alpha$ is a nonnegative rational combination $\delta_{1}, \delta_{2}, \delta_{4}$, then because the support has to be path connected, it 
is actually a nonnegative rational combination of $\delta_{2}, \delta_{4}$. Similarly, if $\alpha$ is a nonnegative rational combination of $\delta_{2}, \delta_{3}, \delta_{4}$, then it must be in fact a nonnegative rational combination of $\delta_{2}$ and $\delta_{4}$.

Now it easily follows that the set of $\sigma$-stable dimension vectors is

$$
\delta_{1}, \quad \delta_{2}, \quad \delta_{3}, \quad \delta_{4}, a \delta_{2}+b \delta_{4}(a, b>0, a, b \in \mathbb{Z}, a \leq 2 b, b \leq 2 a) .
$$

The cone $\bar{\Sigma}(Q, \alpha)$ is a cone over a square. In the diagram below, the coordinates should be interpreted as projective coordinates.

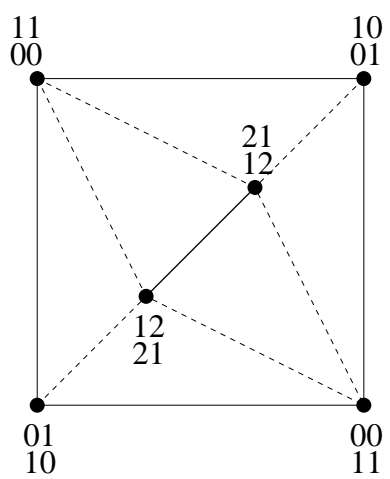

The fat line in the middle of the square corresponds to the imaginary $\sigma$-stable dimension vectors. The dashed lines distinguish the regions where the $\sigma$-stable decomposition looks different.

Some examples of $\sigma$ stable decomposition are:

$$
\begin{gathered}
\begin{array}{cc}
5 & 4 \\
3 & 4
\end{array}=\begin{array}{llll}
1 & 1 \\
0 & 0 & + & 3 \\
3 & 4
\end{array} \\
1 \\
5
\end{gathered}
$$

6.2. $\sigma$-stable decomposition for quivers with oriented cycles. Doubling of the quiver, reduces the $\sigma$-stable decomposition for quivers with oriented cycles, to the case of quivers without oriented cycles. Suppose that $Q$ is a quiver with oriented cycles. We define a new quiver $\widehat{Q}$ by $\widehat{Q}_{0}=Q_{0} \times\{0,1\}$. For every $a \in Q_{1}$ we define an arrow $\widehat{a} \in \widehat{Q}_{1}$ with $t \widehat{a}=(t a, 0)$ and $h \widehat{a}=(h a, 1)$ and for every $x \in Q_{0}$ we define an arrow $\widehat{x} \in \widehat{Q}_{1}$ with $t \widehat{x}=(x, 0)$ and $h \widehat{x}=(h x, 1)$. For example, if $Q$ is the quiver

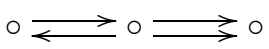

then $\widehat{Q}$ is the quiver

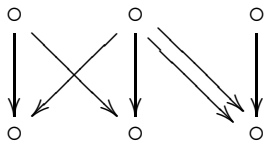

For a $Q$-dimension vector $\alpha$, we define a dimension vector $\widehat{\alpha}$ of $\widehat{Q}$ by $\widehat{\alpha}(x, 0)=\widehat{\alpha}(x, 1)=\alpha(x)$ for all $x \in Q_{0}$. Similarly, if $\sigma$ is a weight of $Q$, we define a weight $\widehat{\sigma}$ of $\widehat{Q}$ by $\widehat{\sigma}(x, 0)=\widehat{\sigma}(x, 1)=\sigma(x)$. We define the weight $\tau$ of $\widehat{Q}$ by $\tau(x, 0)=1$ and $\tau(x, 1)=-1$ for all $x \in Q_{0}$. Note that for any $\alpha \in Q_{0}, \widehat{\alpha}$ is $\tau$-stable.

Proposition 6.7. Suppose that $\alpha$ is a dimension vector and $\sigma$ is a weight for $Q$. Then $\alpha$ is $\sigma$-semistable (stable) if and only if for some large positive integer $m, \widehat{\alpha}$ is $\widehat{\sigma}+m \tau$-semi-stable (stable). 
Proof. Suppose that $\alpha$ is $\sigma$-semi-stable. If $\gamma \hookrightarrow \widehat{\alpha}$ for some $\widehat{Q}$-dimension vector $\gamma$. Note that $\gamma(x, 0) \leq$ $\gamma(x, 1)$ for all $x \in Q_{0}$ because $\widehat{\alpha}(x, 0)=\widehat{\alpha}(x, 1)$, and for a general representation $V$ of dimension $\widehat{\alpha}$ the map $V(\widehat{x}): V(x, 0) \rightarrow V(x, 1)$ is injective. If $\gamma(x, 0)=\gamma(x, 1)$ for all $x \in Q_{0}$ then $\gamma$ is of the form $\widehat{\beta}$ and $\beta \hookrightarrow \alpha$. Then we have $\widehat{\sigma}(\gamma)=\sigma(\beta) \leq 0$. Also we have $(\widehat{\sigma}+m \tau)(\gamma)=\widehat{\sigma}(\gamma) \leq 0$.

Suppose that $\gamma(x, 0)<\gamma(x, 1)$ for some $x \in Q_{0}$. Then $\tau(\gamma)<0$ so in particular for $m$ large enough we will have $(\widehat{\sigma}+m \tau)(\gamma)<0$.

Since there are only finitely many subdimension vectors $\gamma$, we can choose $m$ large enough such that $(\widehat{\sigma}+m \tau)(\gamma) \leq 0$ for all $\gamma \hookrightarrow \widehat{\alpha}$. This shows that $\widehat{\alpha}$ is $(\widehat{\sigma}+m \tau)$-semi-stable.

Conversely, assume that $\widehat{\alpha}$ is $(\widehat{\sigma}+m \tau)$-semi-stable for some $m$ and $\beta \hookrightarrow \alpha$. Then $\widehat{\beta} \hookrightarrow \widehat{\alpha}$, so $0 \geq(\widehat{\sigma}+m \tau)(\widehat{\beta})=\widehat{\sigma}(\widehat{\beta})=\sigma(\beta)$. This shows that $\alpha$ is $\sigma$-semi-stable.

A similar statement with stable instead of semi-stable is easy to prove.

Suppose now that $Q$ is quiver, possibly with oriented cycles. Let us consider the 0 -stable decomposition. Clearly, every representation of $Q$ is 0 -semi-stable in the sense of Theorem 2.41 A representation $V$ is 0 -stable if the only subrepresentations are 0 and $V$ itself. In other words, 0 -stable representations are exactly simple representations. Notice that if there exists an $\alpha$-dimensional simple representation, then the general representation of dimension $\alpha$ is simple. We will call such dimension vectors simple.

Corollary 6.8. Suppose that $Q$ is an arbitrary quiver. For each $x \in Q_{0}$ we define a dimension vector $\delta_{x}$ by $\delta_{x}(y)=0$ for $y \neq x$ and $\delta_{x}(x)=1$. A dimension vector $\alpha$ is simple if

(1) either $\alpha=\delta_{x}$ and $\delta_{x}$ is real (i.e., $\left\langle\delta_{x}, \delta_{x}\right\rangle=1$ );

(2) or $\left\langle\delta_{x}, \alpha\right\rangle \leq 0$ and $\left\langle\alpha, \delta_{x}\right\rangle \leq 0$ for all $x \in Q_{0}$, the full subquiver of $Q$ with vertices

$$
\operatorname{Supp}(\alpha):=\left\{x \in Q_{0} \mid \alpha(x) \neq 0\right\}
$$

is path connected, and if $\alpha$ is isotropic, then $\alpha$ is indivisible.

Remark 6.9. A statement similar to Corollary6.8 was proved in 30. Theorem6.4 is a generalization of this result.

Example 6.10. Consider the quiver

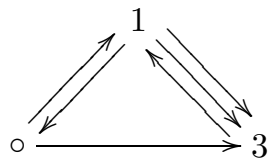

Suppose $\alpha$ that is the dimension vector $\left(a_{1}, a_{2}, a_{3}\right)$. We will find necessary and sufficient conditions for $\alpha$ to be a simple dimension vector. Of course $\alpha$ can be equal to $\delta_{1}, \delta_{2}, \delta_{3}$. The conditions $\left\langle\delta_{i}, \alpha\right\rangle \leq 0$ and $\left\langle\alpha, \delta_{i}\right\rangle \leq 0$ give the inequalities

$$
a_{1} \leq a_{2}+a_{3}, a_{2} \leq a_{1}, a_{3} \leq a_{1}+a_{2}
$$

(other inequalities turn out to be redundant). If $a_{3}=0$ and $a_{1}=a_{2}$, then $\alpha$ is isotropic, so we must have that $a_{1}=a_{2}=1$ in that case. The only support of $\alpha$ which is not possible (because it is not path connected) is $\{2,3\}$, but this is already excluded by the inequalities.

From the inequalities and the remarks above it is easy to deduce that set of simple dimension vectors is given by

$$
\begin{gathered}
(1,0,0),(0,1,0),(0,0,1),(1,0,0),(1,1,0), \text { and all } \\
\left\{\left(a_{1}, a_{2}, a_{3}\right) \in \mathbb{Z}^{3} \mid a_{1} \leq a_{2}+a_{3}, a_{2} \leq a_{1}, a_{3} \leq a_{1}+a_{2}, a_{3}>0\right\}
\end{gathered}
$$


In the picture shows how the simple decomposition looks like. We use projective coordinates.

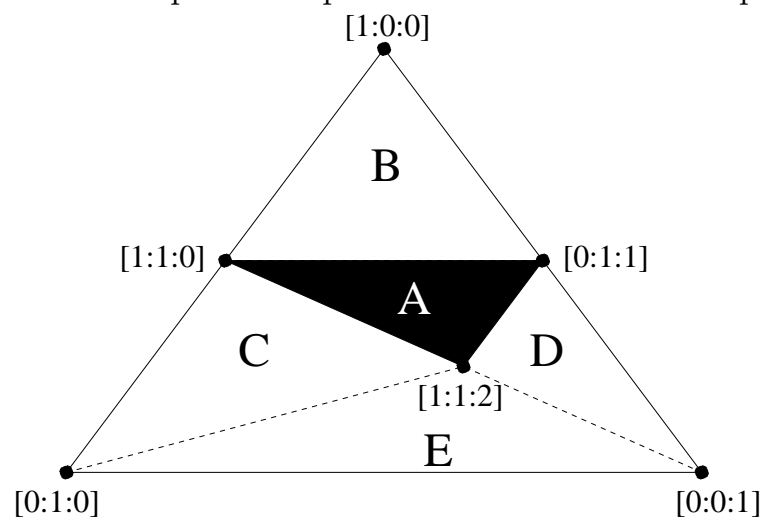

Region $\mathrm{A}$ is defined by $a_{1} \leq a_{2}+a_{3}, a_{2} \leq a_{1}, a_{3} \leq a_{1}+a_{2}$. This will always define a simple dimension vector except when $a_{3}=0$ (and $a_{1}=a_{2}$ ). In that case, the simple decomposition is

$$
(a, a, 0)=a \cdot(1,1,0) .
$$

Region $\mathrm{B}$ is defined by $a_{2}+a_{3} \leq a_{1}$. The simple decomposition in this region is

$$
\begin{aligned}
\left(a_{1}, a_{2}, a_{3}\right)= & \left(a_{1}-a_{2}-a_{3}\right) \cdot(1,0,0) \dot{+}\left(a_{2}+a_{3}, a_{2}, a_{3}\right) \quad \text { if } c_{3}>0 \text { and } \\
& \left(a_{1}, a_{2}, 0\right)=\left(a_{1}-a_{2}\right) \cdot(1,0,0) \dot{+} a_{2} \cdot(1,1,0) .
\end{aligned}
$$

Region $\mathrm{C}$ is defined by $a_{2} \geq a_{1}, 2 a_{1} \geq a_{3}$. The simple decomposition is

$$
\begin{gathered}
\left(a_{1}, a_{2}, a_{3}\right)=\left(a_{2}-a_{1}\right) \cdot(0,1,0) \dot{+}\left(a_{1}, a_{1}, a_{3}\right) \quad \text { if } c_{3}>0 \text { and } \\
\left(a_{1}, a_{2}, 0\right)=\left(a_{2}-a_{1}\right) \cdot(0,1,0) \dot{+} a_{1} \cdot(1,1,0) .
\end{gathered}
$$

Region $\mathrm{D}$ is defined by $a_{1} \geq a_{2}$ and $a_{3} \geq a_{1}+a_{2}$. The simple decomposition here is

$$
\left(a_{1}, a_{2}, a_{3}\right)=\left(a_{3}-a_{1}-a_{2}\right) \cdot(0,0,1) \dot{+}\left(a_{1}, a_{2}, a_{1}+a_{2}\right) .
$$

Region $\mathrm{E}$ is defined by $a_{2} \geq a_{1}, a_{3} \geq 2 a_{1}$. The simple decomposition in this region is

$$
\left(a_{1}, a_{2}, a_{3}\right)=\left(a_{2}-a_{1}\right) \cdot(1,0,0) \dot{+}\left(a_{3}-2 a_{1}\right) \cdot(0,1,0) \dot{+}\left(a_{1}, a_{1}, 2 a_{1}\right) .
$$

Example 6.11. Let $Q$ be the quiver with 3 vertices (labeled 1, 2 and 3), with a loop at each vertex and with arrows $1 \rightarrow 2,2 \rightarrow 3$ and $3 \rightarrow 1$. The set of simple dimension vectors is

$$
(1,0,0),(0,1,0),(0,0,1) \text {, and all }(a, b, c) \text { with } a, b, c>0 \text {. }
$$

Notice that for example a dimension vector of the form $(a, b, 0)(a, b>0)$ is not simple because its support is not path connected.

\section{LitTLEWOOD-RichaRdSON COEFFICIENTS}

7.1. The Klyachko cone. Polynomial representations of $\mathrm{GL}_{n}$ are parametrized by non-increasing integer sequences of length $n$. If $\lambda=\left(\lambda_{1}, \ldots, \lambda_{n}\right)$ is such a sequence, then we denote the corresponding representation by $V_{\lambda}$. We define

$$
|\lambda|:=\lambda_{1}+\cdots+\lambda_{n} .
$$

The Littlewood-Richardson coefficient $c_{\lambda, \mu}^{\nu}$ is defined by

$$
\operatorname{dim}\left(V_{\lambda} \otimes V_{\mu} \otimes V_{\nu}^{\star}\right)^{\mathrm{GL}_{n}} .
$$


We would like to study the set

$$
\mathcal{K}_{n}=\left\{(\lambda, \mu, \nu) \in\left(\mathbb{Z}^{n}\right)^{3} \mid \lambda, \mu, \nu \text { are non-increasing and } c_{\lambda, \mu}^{\nu} \neq 0\right\} .
$$

The cone $\mathbb{R}_{+} \mathcal{K}_{n}$ is the Klyachko cone. The Klyachko cone has dimension $3 n-1$. Let $T_{p, q, r}$ be the quiver with $p+q+r-2$ vertices:

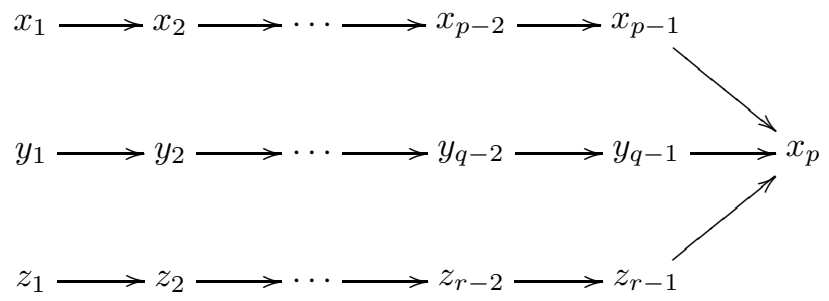

We use the convention $y_{q}=z_{r}=x_{p}$. In [13] we have seen that if we take the dimension vector

$$
\beta=\begin{array}{llll}
1 & 2 & \cdots & n-1 \\
1 & 2 & \cdots & n-1 \\
1 & 2 & \cdots & n-1
\end{array}
$$

for $T_{n, n, n}$, then we can view $\operatorname{dim} \operatorname{SI}(Q, \beta)_{\sigma}$ as a Littlewood-Richardson coefficient as follows. If $\sigma$ is given by

$$
\sigma=\begin{array}{rrrrr}
a_{1} & a_{2} & \cdots & a_{n-1} & \\
b_{1} & b_{2} & \cdots & b_{n-1} & c_{n}, \\
c_{1} & c_{2} & \cdots & c_{n-1}
\end{array}
$$

then

$$
\operatorname{dim} \operatorname{SI}(Q, \beta)_{\sigma}=c_{\lambda, \mu}^{\nu}
$$

where

$$
\begin{aligned}
\lambda & =\lambda(\sigma)=\left(a_{1}+\cdots+a_{n-1}, a_{2}+\cdots+a_{n-1}, \cdots, a_{n-1}, 0\right) \\
\mu & =\mu(\sigma)=\left(b_{1}+\cdots+b_{n-1}, b_{2}+\cdots+b_{n-1}, \cdots, b_{n-1}, 0\right) \\
\nu & =\nu(\sigma)=\left(-c_{n},-\left(c_{n}+c_{n-1}\right), \ldots,-\left(c_{n}+c_{n-1}+\cdots+c_{1}\right)\right)
\end{aligned}
$$

Conversely, if $\lambda, \mu, \nu \in \mathbb{Z}^{n}$, then

$$
c_{\lambda, \mu}^{\nu}=\operatorname{dim} \operatorname{SI}(Q, \beta)_{\sigma}
$$

where

$$
\sigma=\sigma(\lambda, \mu, \nu)=\begin{array}{ccccc}
\lambda_{1}-\lambda_{2} & \lambda_{2}-\lambda_{3}, & \cdots & \lambda_{n-1}-\lambda_{n} & \\
\mu_{1}-\mu_{2} & \mu_{2}-\mu_{3} & \cdots & \mu_{n-1}-\mu_{n} & \lambda_{n}+\mu_{n}-\nu_{1} \\
\nu_{n-1}-\nu_{n} & \nu_{n-2}-\nu_{n-1} & \cdots & \nu_{1}-\nu_{2} &
\end{array}
$$

The set $\Sigma(Q, \beta)$ is almost equal to $\mathcal{K}_{n}$. In fact, we define a bijection

$$
\psi: \Sigma(Q, \beta) \times \mathbb{Z}^{2} \rightarrow \mathcal{K}_{n}
$$

by

$$
\psi(\sigma, a, b)=(\lambda(\sigma)+a \cdot \mathbf{1}, \mu(\sigma)+b \cdot \mathbf{1}, \nu(\sigma)+(a+b) \cdot \mathbf{1}) .
$$

where $\mathbf{1}=(1,1, \ldots, 1) \in \mathbb{N}^{n}$. This bijection extends to an isomorphism of the cones $\mathbb{R}_{+} \Sigma(Q, \beta) \times \mathbb{R}^{2}$ and $\mathbb{R}_{+} \mathcal{K}_{n}$. The inverse of $\psi$ is given by

$$
(\lambda, \mu, \nu) \mapsto\left(\sigma(\lambda, \mu, \nu), \lambda_{n}, \mu_{n}\right) .
$$


Recall that if $\sigma=\langle\alpha, \cdot\rangle$, then $\operatorname{dim} \operatorname{SI}(Q, \beta)_{\sigma}=\alpha \circ \beta$. The numbers $\alpha \circ \beta$ can be interpreted as Littlewood-Richardson coefficients even if $\alpha, \beta$ are arbitrary dimension vectors and $Q=T_{p, q, r}$ where $p, q, r$ are arbitrary. This can be done as follows. Let us define

$$
\widetilde{c}_{\lambda, \mu, \nu}=\widetilde{c}_{\lambda, \mu, \nu}^{(n)}=\operatorname{dim}\left(V_{\lambda} \otimes V_{\mu} \otimes V_{\nu}\right)^{\mathrm{SL}_{n}} .
$$

If $\widetilde{c}_{\lambda, \mu, \nu} \neq 0$, then $|\lambda|+|\mu|+|\nu|$ must be a multiple of $n$, say $m n$. In that case

$$
\widetilde{c}_{\lambda, \mu, \nu}=c_{\lambda, \mu}^{\nu^{\star}}
$$

where

$$
\nu^{\star}=\left(m-\nu_{n}, m-\nu_{n-1}, \ldots, m-\nu_{1}\right) .
$$

Definition 7.1. Let $\underline{x}=\left(x_{1}, x_{2}, \ldots, x_{n}\right), \underline{y}=\left(y_{1}, y_{2}, \ldots, y_{n}\right)$ be two nondecreasing sequences of nonnegative integers. We define a partition $\bar{P}(\underline{x}, \underline{y})$ by

$$
P(\underline{x}, \underline{y})=\left(x_{n-1}^{y_{n-1} y_{n-1}}, x_{n-2}^{y_{n-1}-y_{n-2}}, \ldots, x_{1}^{y_{2}-y_{1}}, 0^{y_{1}}\right) .
$$

Graphically, this partition can be found as follows. In the Euclidean plane we draw a square with vertices $(0,0),\left(x_{n}, 0\right),\left(0, y_{n}\right),\left(x_{n}, y_{n}\right)$. and we plot the points

$$
\left(x_{1}, y_{1}\right),\left(x_{2}, y_{2}\right), \ldots,\left(x_{n}, y_{n}\right) .
$$

We take the region in the square above and left to those points. Viewed in the unit grid, the corresponding partition $P(\underline{x}, \underline{y})$ can be read off.

Example 7.2. If $\underline{x}=(2,4,5,6)$ and $\underline{y}=(1,3,3,4)$ then $P(\underline{x}, \underline{y})=(5,2,2,0)$ by the diagram below:

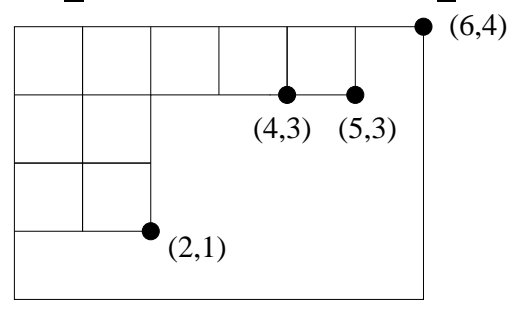

We consider the quiver $T_{p, q, r}$. Let $\alpha$ and $\beta$ be dimension vectors. We write $\alpha(x)=\left(\alpha\left(x_{1}\right), \ldots, \alpha\left(x_{p}\right)\right)$, $\alpha(y)=\left(\alpha\left(y_{1}\right), \ldots, \alpha\left(y_{q}\right)\right)$ and in a similar way we define $\alpha(z), \beta(x), \beta(y), \beta(z)$.

\section{Lemma 7.3.}

$$
\alpha \circ \beta=\widetilde{c}_{\lambda, \mu, \nu}
$$

where

$$
\lambda=P(\alpha(x), \beta(x)), \mu=P(\alpha(y), \beta(y)), \nu=P(\alpha(z), \beta(z)) .
$$

Proof. This is an easy computation, see [13].

Remark 7.4. Note that if $1 / p+1 / q+1 / r>1$, then $T_{p, q, r}$ is a quiver of finite type and for all dimension vectors $\alpha, \beta$ we have $\alpha \circ \beta=0$ or $\alpha \circ \beta=1$. For a partition $\lambda=\left(\lambda_{1}, \ldots, \lambda_{n}\right)$ we define

$$
j(\lambda)=\#\left\{i \mid \lambda_{i+1} \neq \lambda_{i}, 1 \leq i \leq n-1\right\}
$$

If $\lambda$ is trivial then $j(\lambda)=0$, if $\lambda$ is a box then $j(\lambda)=1$ and if $\lambda$ is L-shaped (fat hook) then $j(\lambda)=2$. A coefficient $\widetilde{c}_{\lambda, \mu, \nu}$ can be obtained from the quiver $T_{p, q, r}$ with $p=j(\lambda)+1, q=j(\mu)+1$ and $r=j(\nu)+1$. In particular, we get the corollary below: 
Corollary 7.5. If

$$
\frac{1}{j(\lambda)+1}+\frac{1}{j(\mu)+1}+\frac{1}{j(\nu)+1}>1
$$

then $\widetilde{c}_{\lambda, \mu, \nu}$ equals 0 or 1 .

Example 7.6. Let us consider the quiver $T_{3,3,2}$ :

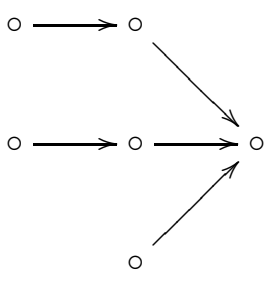

Let $\alpha, \beta$ be the dimension vectors

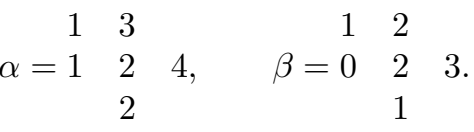

Now $\alpha \circ \beta$ is equal to the LR-coefficient $\widetilde{c}_{\lambda, \mu, \nu}=1$ were $\lambda=(3,1), \mu=(2,1,1)$ and $\nu=(2,2)$.

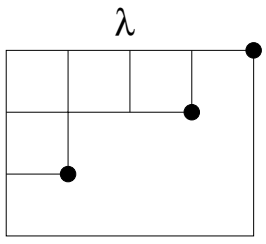

$(3,1)$

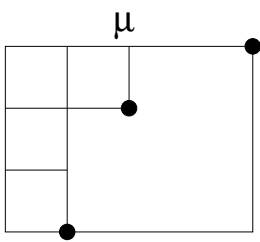

$(2,1,1)$

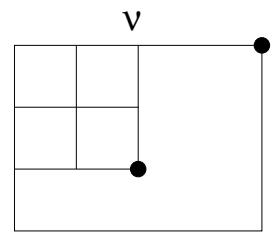

$(2,2)$

7.2. Walls of the Klyachko cone. Let us consider the quiver $Q=T_{n, n, n}$ and the dimension vector

$$
\beta=\begin{array}{llll}
1 & 2 & \cdots & n-1 \\
1 & 2 & \cdots & n-1 \\
1 & 2 & \cdots & n-1
\end{array}
$$

Lemma 7.7. The dimension vector $\beta$ above is a Schur root.

Proof. For $n \leq 2$ this is an easy check that $\beta$ is a real Schur root. For $n \geq 3$ the dimension vector $\beta$ is indivisible and lies in the fundamental set (see [23, §1]). This implies that $\beta$ is a Schur root by [23, Theorem B (d)]).

We will study the cone $\mathbb{R}_{+} \Sigma(Q, \beta)$ which is essentially the Klyachko cone. The following Theorem is equivalent to a result of Knutson, Tao and Woodward (see 29]). It gives a precise description of the walls of the Klyachko cone.

Theorem 7.8. For every pair $\left(\beta_{1}, \beta_{2}\right)$ with $\beta=\beta_{1}+\beta_{2}, \beta_{1}, \beta_{2}$ nondecreasing along arms, $\beta_{1} \circ \beta_{2}=1$ the inequality $\sigma\left(\beta_{1}\right) \leq 0$ defines a wall of $\mathbb{R}_{+} \Sigma(Q, \beta)$. All nontrivial walls can be uniquely obtained this way.

Proof. Clearly $\beta_{1}$ and $\beta_{2}$ have at most jumps 1 along the arms of the quiver $Q$. It follows from Lemma 7.7 that $\beta_{1}, \beta_{2}$ are Schur roots. Now either $\operatorname{ext}_{Q}\left(\beta_{2}, \beta_{1}\right)=0$ or $\operatorname{hom}_{Q}\left(\beta_{2}, \beta_{1}\right)=0$ by Theorem 2.38 The first would give a nontrivial decomposition of $\beta$, therefore $\operatorname{hom}_{Q}\left(\beta_{2}, \beta_{1}\right)=0$ and $\beta_{1}, \beta_{2}$ is a quiver Schur sequence. This shows that $\sigma\left(\beta_{1}\right) \leq 0$ defines a wall by Theorem 5.1. 
For every wall, there exists a Schur sequence $\left(\beta_{1}, \beta_{2}\right)$ such that that $\beta=c_{1} \beta_{1}+c_{2} \beta_{2}$ with $c_{i}$ positive integers and the wall is defined by $\sigma\left(\beta_{1}\right) \leq 0$ by Theorem 5.1. Note that for $T_{n, n, n}$ a Schur root either has support on one arm (in which case it corresponds to a positive root of $A_{n-1}$ ), or it is nondecreasing along each arm. Because $\beta_{1} \hookrightarrow \beta$, it is easy to see that $\beta_{1}$ must also be nondecreasing. Indeed, for a general representation of dimension $\beta$, the linear maps along the arms are injective, so these maps are also injective for every subrepresentation. But $\beta_{2}$ could have support on one arm. In that case it follows from $\left\langle\beta_{1}, \beta_{2}\right\rangle=0$ that $\beta_{2}$ is simple. The inequalities following from such a quiver sequence are trivial, they say that the partitions $\lambda, \mu$ and $\nu$ must be weakly decreasing. If $\beta_{1}$ and $\beta_{2}$ are both nondecreasing along the arms, then $c_{1}=c_{2}=1$ because $\beta=\beta_{1}+\beta_{2}$ and $\beta$ jumps only by steps of 1 along the arms.

We need to find all $\beta_{1}, \beta_{2}$ such that $\beta=\beta_{1}+\beta_{2}$ and $\beta_{1} \circ \beta_{2}=1$. In that case $\sigma\left(\beta_{1}\right) \leq 0$ defines a wall of $\Sigma(Q, \beta)$. If we are dealing with a nontrivial wall, i.e., not $\lambda_{i} \geq \lambda_{i+1}, \mu_{i} \geq \mu_{i+1}$ or $\nu_{i} \geq \nu_{i+1}$ for some $i$, then both $\beta_{1}$ and $\beta_{2}$ are increasing along the arms, with jumps at most 1 . The fact that $\beta_{1}$ has jumps of at most 1 , gives these inequalities a special form, namely, if we take

$$
\begin{aligned}
& I=\left\{i \mid \beta_{1}\left(x_{i-1}\right)=\beta_{1}\left(x_{i}\right), 1 \leq i \leq n\right\}, \\
& J=\left\{i \mid \beta_{1}\left(y_{i-1}\right)=\beta_{1}\left(y_{i}\right), 1 \leq i \leq n\right\}, \\
& K=\left\{i \mid \beta_{1}\left(z_{i-1}\right)=\beta_{1}\left(z_{i}\right), 1 \leq i \leq n\right\},
\end{aligned}
$$

(by convention $\beta_{1}\left(x_{0}\right)=\beta_{1}\left(y_{0}\right)=\beta_{1}\left(z_{0}\right)=0$ ), then the inequality corresponding to $\beta_{1}$ is

$$
\sum_{i \in I} \lambda_{i}+\sum_{i \in J} \mu_{i} \leq \sum_{i \in K} \nu_{n-i}
$$

Note that $\# I=\# J=\# K=\beta_{1}\left(x_{n}\right)$. Now (20) is a necessary inequality for the Klyachko cone if $\beta_{1} \circ \beta_{2}=1$. If $\beta_{1} \circ \beta_{2}>0$ then (20) still defines a true inequality. Now $\beta_{1} \circ \beta_{2}$ is the value of a Littlewood-Richardson coefficient for $\mathrm{SL}_{\beta_{2}\left(x_{n}\right)}$. Since $\beta_{2}\left(x_{n}\right)<n$ we know necessary and sufficient inequalities for the corresponding LR-coefficient to be nonzero. This explains the inductive nature of the Klyachko inequalities.

Example 7.9. Consider the quiver $T_{3,3,3}$ and

$$
\beta=\begin{array}{lll}
1 & 2 \\
1 & 2 & 3 . \\
1 & 2
\end{array}
$$

The LR-coefficient $c_{\lambda, \mu}^{\nu}$ corresponds to $\operatorname{dim} \operatorname{SI}(Q, \beta)_{\sigma}$ where $\sigma$ is given by

$$
\begin{aligned}
& \begin{array}{ll}
\lambda_{1}-\lambda_{2} & \lambda_{2}-\lambda_{3}
\end{array} \\
& \sigma=\mu_{1}-\mu_{2} \quad \mu_{2}-\mu_{3} \quad \lambda_{3}+\mu_{3}-\nu_{1} . \\
& \nu_{2}-\nu_{3} \quad \nu_{1}-\nu_{2}
\end{aligned}
$$

For example the sequence

$$
\begin{array}{llllll}
1 & 2 & & 0 & 0 & \\
0 & 1 & 2, & 1 & 1 & 1 \\
0 & 1 & & 1 & 1 &
\end{array}
$$

is a Schur sequence, because $\widetilde{c}_{2,0,0}^{1}=c_{2,0}^{2}=1$. This Schur sequence corresponds to the wall

$$
\lambda_{1}+\lambda_{2}+\mu_{2}+\mu_{3} \leq \nu_{1}+\nu_{2}
$$

By permuting the arms, we get the inequalities

$$
\begin{aligned}
& \lambda_{2}+\lambda_{3}+\mu_{1}+\mu_{2} \leq \nu_{1}+\nu_{2}, \\
& \lambda_{2}+\lambda_{3}+\mu_{2}+\mu_{3} \leq \nu_{2}+\nu_{3} .
\end{aligned}
$$


Other walls are given by the Schur sequences

$$
\begin{array}{llrrrrl}
1 & 1 & 0 & 1 & & \\
1 & 1 & 2, & 0 & 1 & 1 & \left(\widetilde{c}_{1,1,0}^{(1)}=c_{1,1}^{2}=1\right) .
\end{array}
$$

By permuting arms we get the inequalities

$$
\begin{aligned}
& \lambda_{1}+\lambda_{3}+\mu_{1}+\mu_{3} \leq \nu_{1}+\nu_{2}, \\
& \lambda_{1}+\lambda_{3}+\mu_{2}+\mu_{3} \leq \nu_{1}+\nu_{3}, \\
& \lambda_{2}+\lambda_{3}+\mu_{1}+\mu_{3} \leq \nu_{1}+\nu_{3} . \\
& \begin{array}{llll}
1 & 1 & 0 & 1
\end{array} \\
& \begin{array}{lllllll}
0 & 0 & 1,1 & 2 & 2 & \left(\widetilde{c}_{11,0,0}^{(2)}=c_{11,0}^{11}=1\right)
\end{array} \\
& \begin{array}{llll}
0 & 0 & 1 & 2
\end{array}
\end{aligned}
$$

gives the inequalities

$$
\begin{aligned}
& \lambda_{1}+\mu_{3} \leq \nu_{1} \\
& \lambda_{3}+\mu_{1} \leq \nu_{1} \\
& \lambda_{3}+\mu_{3} \leq \nu_{3}
\end{aligned}
$$

The Schur sequence

$$
\begin{array}{rrrrll}
0 & 1 & 1 & 1 & & \\
0 & 1 & 1, & 1 & 1 & 2 \\
0 & 0 & 1 & 2
\end{array} \quad\left(\widetilde{c}_{1,1,0}^{(2)}=c_{1,1}^{11}=1\right)
$$

gives the inequalities

$$
\begin{aligned}
& \lambda_{2}+\mu_{2} \leq \nu_{1} \\
& \lambda_{2}+\mu_{3} \leq \nu_{2} \\
& \lambda_{3}+\mu_{2} \leq \nu_{2}
\end{aligned}
$$

Besides these, there are 6 trivial walls corresponding to the inequalities $\lambda_{1} \geq \lambda_{2} \geq \lambda_{3}, \mu_{1} \geq \mu_{2} \geq \mu_{3}$ and $\nu_{1} \geq \nu_{2} \geq \nu_{3}$. The Schur sequence

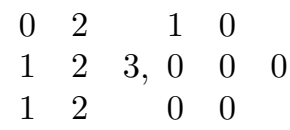

implies the inequalities

$$
\begin{aligned}
& \lambda_{1} \geq \lambda_{2}, \\
& \mu_{1} \geq \mu_{2}, \\
& \nu_{2} \geq \nu_{3} .
\end{aligned}
$$

The Schur sequence

$$
\begin{array}{rrrrrr}
1 & 1 & & 0 & 1 & \\
1 & 2 & 3, & 0 & 0 & 0 \\
1 & 2 & & 0 & 0 &
\end{array}
$$

leads to the inequalities

$$
\begin{aligned}
& \lambda_{3} \geq \lambda_{3} \\
& \mu_{2} \geq \mu_{3} \\
& \nu_{1} \geq \nu_{2}
\end{aligned}
$$


7.3. Faces of the Klyachko cone of arbitrary codimension. For the quiver $Q=T_{n, n, n}$, Theorem 5.1 translates to:

Corollary 7.10. There is a 1-1 correspondence between the faces of codimension $l$ of $\mathcal{K}_{n}$ and $S c h u r$ sequences $\left(\beta_{1}, \ldots, \beta_{l+1}\right)$ such that $\beta=c_{1} \beta_{1}+\cdots+c_{l+1} \beta_{l+1}$ for some positive integers $c_{1}, \ldots, c_{l+1}$, $\beta_{1}, \ldots, \beta_{l+1}$ are Schur roots, and $\beta_{i} \circ \beta_{j}=1$ for all $i<j$.

However, If $l>1$ then the $c_{i}$ may be larger than 1 . There is no easy criterion for a dimension vector to be a Schur root, but a fast algorithm for determining whether a dimension vector is a Schur root was given in 14. This makes it more difficult to find the faces of higher codimension. Still, we obtain some interesting features.

Corollary 7.11. Suppose that $(\lambda, \mu, \nu)$ lies in a face $F$ of $\mathcal{K}_{n}$ of codimension l. Let $j(\lambda), j(\mu), j(\nu)$ be the number of jumps in $\lambda, \mu, \nu$ respectively.

(a)

$$
j(\lambda)+j(\mu)+j(\nu) \leq 4 n-4-l,
$$

(b) if $\widetilde{c}_{\lambda, \mu, \nu}>1$ then

$$
j(\lambda)+j(\mu)+j(\nu) \leq 4 n-6-l .
$$

Proof. Let $\beta$ be the usual dimension vector for $T_{n, n, n}$. Now $\alpha \circ \beta=\widetilde{c}_{\lambda, \mu, \nu}$ for some dimension vector $\alpha$. Let $\sigma=\langle\alpha, \cdot\rangle$. Because $\sigma$ is in a face of $\Sigma(Q, \beta)$ of codimension $l$, exactly $l+1$ distinct dimension vectors appear in the $\sigma$-stable decomposition of $\beta$. Suppose that the $\sigma$-stable decomposition of $\beta$ is

$$
\beta=c_{1} \cdot \beta_{1} \dot{+} c_{2} \cdot \beta_{2} \cdots \dot{+} c_{l+1} \cdot \beta_{l+1} .
$$

Whenever $\beta_{i}\left(x_{n}\right)=0$, then $\beta_{i}$ has support on one arm and the equation $\sigma\left(\beta_{i}\right)=0$ corresponds to the equation $\lambda_{j}=\lambda_{k}, \mu_{j}=\mu_{k}$ or $\nu_{j}=\nu_{k}$ for some $j \neq k$. Notice also that all $\beta_{j}$ 's correspond to linearly independent equations. It now follows that

$$
j(\lambda)+j(\mu)+j(\nu)+\#\left\{i \mid \beta_{i}\left(x_{n}\right)=0\right\} \leq 3 n-3 .
$$

Since $\sum_{j} c_{j} \beta_{j}\left(x_{n}\right)=n$, we have $\#\left\{j \mid \beta_{j}\left(x_{n}\right)>0\right\} \leq n$ and $\#\left\{j \mid \beta_{j}\left(x_{n}\right)=0\right\} \geq l+1-n$. Now (a) follows.

If $\widetilde{c}_{\lambda, \mu, \nu}>1$, then at least one of the $\beta_{i}$ 's is an imaginary Schur root, so $\beta_{i}\left(x_{n}\right) \geq 3$. This shows that $\#\left\{j \mid \beta_{j}\left(x_{n}\right)>0\right\} \leq n-2$ and (b) follows.

The cone $\mathbb{R}_{+} \Sigma(Q, \beta)$ has one 0 -dimensional face, namely $\{0\}$. This corresponds to the the 2 dimensional face of $\mathbb{R}_{+} \mathcal{K}_{n}$ consisting of all $(\lambda, \mu, \nu), \lambda=(a, \ldots, a), \mu=(b, \ldots, b), \nu=(a+b, \ldots, a+b)$. It would be also interesting to study the extremal rays of the cone $\mathbb{R}_{+} \Sigma(Q, \beta)$ (or equivalently the 3 -dimensional faces of $\left.\mathbb{R}_{+} \mathcal{K}_{n}\right)$. They span the cone $\Sigma(Q, \beta)$ (or the Klyachko cone). The codimension of the extremal rays is $3 n-4$. One interesting question is, whether $c_{\lambda, \mu}^{\nu}=1$ whenever $(\lambda, \mu, \nu)$ is on an extremal ray of $\mathbb{R}_{+} \mathcal{K}_{n}$ (here by extremal ray we mean a 3 -dimensional face of $\mathcal{K}_{n}$ ). We first give a positive result in this direction:

Corollary 7.12. If $(\lambda, \mu, \nu)$ is in an extremal ray of $\mathbb{R}_{+} \mathcal{K}_{n}$, and $n \leq 7$, then $c_{\lambda, \mu}^{\nu}=1$.

Proof. Suppose that $c_{\lambda, \mu}^{\nu}>1$. Then $\widetilde{c}_{\lambda, \mu, \nu^{\star}}=c_{\lambda, \mu}^{\nu}>1$ for some partition $\nu^{\star}$. Then $j(\lambda)+j(\mu)+$ $j\left(\nu^{\star}\right) \geq 6$ (this follows from Remark 7.4). So

$$
6 \leq 4 n-6-(3 n-4)
$$

by Corollary 7.11 and we deduce that $n \geq 8$. Contradiction. 
Example 7.13. We study $T_{8,8,8}$ with the weight

$$
\sigma=\begin{array}{llllllll}
1 & 0 & 0 & 0 & 1 & 0 & 0 & \\
0 & 0 & 1 & 0 & 0 & 1 & 0 & -3 \\
0 & 0 & 1 & 0 & 0 & 1 & 0 &
\end{array}
$$

The $\sigma$-stable decomposition of

$$
\beta=\begin{array}{rrrrrrrr}
1 & 2 & 3 & 4 & 5 & 6 & 7 & \\
1 & 2 & 3 & 4 & 5 & 6 & 7 & 8
\end{array}
$$

is

$$
\begin{aligned}
& \begin{array}{llllllllllllll}
1 & 1 & 1 & 1 & 2 & 2 & 2 & 0 & 0 & 0 & 0 & 1 & 1 & 1
\end{array} \\
& \begin{array}{llllllllllllllllll}
0 & 0 & 1 & 1 & 1 & 2 & 2 & 3 & \dot{+} & 0 & 0 & 1 & 1 & 1 & 1 & 1 & 1 \dot{+}
\end{array} \\
& \begin{array}{lllllllllllllll}
0 & 0 & 1 & 1 & 1 & 2 & 2 & 0 & 0 & 0 & 0 & 0 & 0 & 0
\end{array} \\
& \begin{array}{rrrrrrrrrrrrrr}
0 & 0 & 0 & 0 & 1 & 1 & 1 & 0 & 0 & 0 & 0 & 0 & 0 & 0
\end{array} \\
& \begin{array}{lllllllllllllllll}
0 & 0 & 0 & 0 & 0 & 0 & 0 & 1 & \dot{+} & 0 & 0 & 0 & 0 & 0 & 1 & 1 & 1 \dot{+}
\end{array} \\
& \begin{array}{lllllllllllllll}
0 & 0 & 1 & 1 & 1 & 1 & 1 & 0 & 0 & 1 & 1 & 1 & 1 & 1
\end{array} \\
& \begin{array}{rrrrrrrrrrrrrr}
0 & 0 & 0 & 0 & 0 & 0 & 0 & 0 & 0 & 0 & 0 & 1 & 1 & 1
\end{array} \\
& \begin{array}{lllllllllllllllll}
0 & 0 & 1 & 1 & 1 & 1 & 1 & 1 & \dot{+} & 0 & 0 & 0 & 0 & 0 & 1 & 1 & 1 \\
+
\end{array} \\
& \begin{array}{lllllllllllllll}
0 & 0 & 0 & 0 & 0 & 1 & 1 & 0 & 0 & 0 & 0 & 0 & 1 & 1
\end{array} \\
& \delta_{x_{2}} \dot{+} 2 \cdot \delta_{x_{3}} \dot{+} 3 \cdot \delta_{x_{4}}+\delta_{x_{6}}+2 \cdot \delta_{x_{7}} \dot{+} \\
& \delta_{y_{1}} \dot{+} 2 \cdot \delta_{y_{2}} \dot{+} \delta_{y_{4}} \dot{+} 2 \cdot \delta_{y_{5}} \dot{+} \delta_{y_{7}} \dot{+} \\
& \delta_{z_{1}} \dot{+} 2 \cdot \delta_{z_{2}} \dot{+} \delta_{z_{4}} \dot{+} 2 \cdot \delta_{z_{5}} \dot{+} \delta_{z_{7}} .
\end{aligned}
$$

Here for any vertex $p$ of the quiver $Q=T_{8,8,8} \delta_{p}$ denotes the dimension vector of the simple representation corresponding to the vertex $p$. In the $\sigma$-stable decomposition of $\beta$, there are 21 distinct Schur roots. The quiver $T_{8,8,8}$ has 22 vertices, so this proves that $\sigma$ is in an extremal ray of $\Sigma(Q, \beta)$. The LR-Richardson coefficient corresponding to $\beta$ and $\sigma$ is $\widetilde{c}_{\lambda, \mu, \nu}$ where

$$
\lambda=(2,1,1,1,1,0,0,0), \mu=(2,2,2,1,1,1,0,0), \nu=(2,2,2,1,1,1,0,0) .
$$

The value of $\widetilde{c}_{\lambda, \mu, \nu}$ is 2 . In fact, for any $N$ we have $\widetilde{c}_{N \lambda, N \mu, N \nu}=N+1$.

7.4. A multiplicative formula for Littlewood-Richardson coefficients. Let $\beta$ and $T_{n, n, n}$ as before.

Theorem 7.14. Suppose $\beta=\beta_{1}+\beta_{2}$ and $\beta_{1} \circ \beta_{2}=1$. Let $\alpha$ be another dimension vector with $\alpha \circ \beta=\widetilde{c}_{\lambda, \mu, \nu}$. Put $\sigma=\langle\alpha, \cdot\rangle$. The inequality $\sigma\left(\beta_{1}\right) \leq 0$ translates to

$$
\sum_{i \in I} \lambda_{i}+\sum_{i \in J} \mu_{i} \leq \sum_{i \in K} \nu_{i}
$$

where $I, J, K$ are subsets of $S=\{1,2, \ldots, n\}$ of the same cardinality. Suppose that equality in (21) holds for $(\lambda, \mu, \nu) \in\left(\mathbb{Z}^{n}\right)^{3}$. Define

$$
\begin{gathered}
\lambda^{*}=\left(\lambda_{i_{1}}, \cdots, \lambda_{i_{r}}\right), \quad I=\left\{i_{1}, i_{2}, \ldots, i_{r}\right\}, \\
\lambda^{\#}=\left(\lambda_{\widetilde{1}_{1}}, \cdots, \lambda_{\widetilde{1}_{n-r}}\right), \quad S \backslash I=\left\{\widetilde{1}_{1}, \widetilde{1}_{2}, \ldots, \widetilde{1}_{n-r}\right\}, \\
\mu^{*}=\left(\mu_{j_{1}}, \cdots, \mu_{j_{r}}\right), \quad J=\left\{j_{1}, j_{2}, \ldots, j_{r}\right\}, \\
\mu^{\#}=\left(\mu_{\widetilde{\jmath}_{1}}, \cdots, \mu_{\widetilde{\jmath}_{n-r}}\right), \quad S \backslash J=\left\{\widetilde{\mathrm{J}}_{1}, \widetilde{\mathrm{J}}_{2}, \ldots, \widetilde{\mathrm{J}}_{n-r}\right\}, \\
\nu^{*}=\left(\nu_{k_{1}}, \cdots, \nu_{j_{r}}\right), \quad K=\left\{k_{1}, k_{2}, \ldots, k_{r}\right\}, \\
\nu^{\#}=\left(\nu_{\widetilde{k}_{1}}, \cdots, \nu_{\widetilde{k}_{n-r}}\right), \quad S \backslash K=\left\{\widetilde{k}_{1}, \widetilde{k}_{2}, \ldots, \widetilde{k}_{n-r}\right\} .
\end{gathered}
$$


Then we have

$$
c_{\lambda, \mu}^{\nu}=c_{\lambda^{*}, \mu^{*}}^{\nu^{*}} c_{\lambda^{\#}, \mu^{\#}}^{\nu^{\#}} .
$$

Proof. If $\sigma$ is in the interior of the wall, then the $\sigma$-stable decomposition of $\beta$ is $\beta_{1} \dot{+} \beta_{2}$ and by Theorem 3.20 we get

$$
c_{\lambda, \mu}^{\nu}=\alpha \circ \beta=\left(\alpha \circ \beta_{1}\right)\left(\alpha \circ \beta_{2}\right)=c_{\lambda^{*}, \mu^{*}}^{\nu^{*}} c_{\lambda^{\#}, \mu^{\#}}^{\nu^{\#}} .
$$

Assume $\sigma$ is not in the interior of the wall. Suppose that the $\sigma$-stable decomposition of $\beta_{1}$ is

$$
c_{1} \cdot \gamma_{1} \dot{+} \cdots \dot{+} c_{r} \cdot \gamma_{r}
$$

and that the $\sigma$-stable decomposition of $\beta_{2}$ is

$$
d_{1} \cdot \delta_{1} \dot{+} \cdots \dot{+} d_{s} \cdot \delta_{s}
$$

Then the $\sigma$-stable decomposition of $\beta$ is

$$
c_{1} \cdot \gamma_{1} \dot{+} \cdots \dot{+} c_{r} \cdot \gamma_{r} \dot{+} d_{1} \cdot \delta_{1} \dot{+} \cdots \dot{+} d_{s} \cdot \delta_{s} .
$$

Note that $\left\{\gamma_{1}, \ldots, \gamma_{r}\right\}$ and $\left\{\delta_{1}, \ldots, \delta_{s}\right\}$ are disjoint because $\gamma_{i} \Perp \delta_{j}$ for all $i, j$. By Theorem 3.20 we get

$$
c_{\lambda, \mu}^{\nu}=\alpha \circ \beta=\prod\left(\alpha \circ\left(c_{i} \gamma_{i}\right)\right) \prod\left(\alpha \circ\left(d_{i} \delta_{i}\right)\right)=\left(\alpha \circ \beta_{1}\right)\left(\alpha \circ \beta_{2}\right)=c_{\lambda^{*}, \mu^{*}}^{\nu^{*}} \nu_{\lambda, \mu}^{\nu_{\#}^{\#}} .
$$

Example 7.15. For $n=8, \beta=\beta_{1}+\beta_{2}$ with

$$
\begin{aligned}
& \begin{array}{rrrrrrrrrrrrrr}
1 & 1 & 2 & 2 & 3 & 3 & 4 & 0 & 1 & 1 & 2 & 2 & 3 & 3
\end{array} \\
& \beta_{1}=1 \quad 1 \quad 2 \quad 2 \quad 3 \quad 3 \quad 3 \quad 4 \quad 5, \beta_{2}=0 \begin{array}{llllllll}
0 & 1 & 1 & 2 & 2 & 3 & 3 & 3
\end{array} \\
& \begin{array}{llllllllllllll}
0 & 0 & 1 & 2 & 3 & 3 & 4 & 1 & 2 & 2 & 2 & 2 & 3 & 3
\end{array}
\end{aligned}
$$

Now $\beta_{1} \circ \beta_{2}=\widetilde{c}_{321,321,3}^{(3)}=c_{321,321}^{552}$, so $\beta_{1}, \beta_{2}$ is a Schur sequence. The corresponding inequality for the Klyachko cone is

$$
\lambda_{1}+\lambda_{3}+\lambda_{5}+\lambda_{7}+\lambda_{8}+\mu_{1}+\mu_{3}+\mu_{5}+\mu_{7}+\mu_{8} \leq \nu_{1}+\nu_{2}+\nu_{4}+\nu_{5}+\nu_{6} .
$$

or equivalently

$$
\lambda_{2}+\lambda_{4}+\lambda_{6}+\mu_{2}+\mu_{4}+\mu_{6} \geq \nu_{3}+\nu_{7}+\nu_{8} .
$$

If now these inequalities are equalities, then

$$
c_{\lambda, \mu}^{\nu}=c_{\lambda^{*}, \mu^{*}}^{\nu^{*}} c_{\lambda^{\#}, \mu^{\#}}^{\nu^{\#}}
$$

where

$$
\lambda^{*}=\left(\lambda_{1}, \lambda_{3}, \lambda_{5}, \lambda_{7}, \lambda_{8}\right), \mu^{*}=\left(\mu_{1}, \mu_{3}, \mu_{5}, \mu_{7}, \mu_{8}\right), \nu^{*}=\left(\nu_{1}, \nu_{2}, \nu_{4}, \nu_{5}, \nu_{6}\right)
$$

and

$$
\lambda^{\#}=\left(\lambda_{2}, \lambda_{4}, \lambda_{6}\right), \mu^{\#}=\left(\mu_{2}, \mu_{4}, \mu_{6}\right), \nu^{\#}=\left(\nu_{3}, \nu_{7}, \nu_{8}\right) .
$$

For example, take $\lambda=\mu=(8,4,4,2,2,0,0,0), \nu=(10,8,7,4,3,3,3,2)$.

Then $c_{\lambda, \mu}^{\nu}=c_{\lambda^{*}, \mu^{*}}^{\nu^{*}} c_{\lambda^{\#}, \mu^{\#}}^{\nu^{\#}}$ where $\lambda^{*}=\mu^{*}=(8,4,2,0,0), \nu^{*}=(10,8,4,3,3), \lambda^{\#}=\mu^{\#}=(4,2,0)$, $\nu^{*}=(-7,3,2)$. Indeed, $c_{\lambda^{*}, \mu^{*}}^{\nu^{*}}=5, c_{\lambda^{\#}, \mu^{\#}}^{\nu^{\#}}=2$ and $c_{\lambda, \mu}^{\nu}=10$. 


\section{Appendix: BelKale's proof of Fulton's COnjecture}

At the AMS Summer Institute on Algebraic Geometry Meeting in Seattle, Belkale explained his geometric proof of Theorem 1.6 to the first author and how it generalizes to the more general quiver setting. What follows below is a reconstruction of that proof. We are grateful to Prakash Belkale for letting us include his proof in our paper.

For a nonnegative integer $d$, the Grassmannian of $d$-dimensional subspaces of the $n$-dimensional vector space $V$ is denoted by

$$
\operatorname{Gr}\left(\begin{array}{l}
V \\
d
\end{array}\right) \text {. }
$$

Suppose that $\alpha, \gamma$ are dimension vectors for a quiver $Q$. We define

$$
\begin{aligned}
\operatorname{Gr}\left(\begin{array}{c}
\alpha \\
\gamma
\end{array}\right) & =\prod_{x \in Q_{0}} \operatorname{Gr}\left(\begin{array}{c}
K^{\alpha(x)} \\
\gamma(x)
\end{array}\right) . \\
\operatorname{Hom}\left(K^{\alpha}, K^{\beta}\right) & =\prod_{x \in Q_{0}} \operatorname{Hom}\left(K^{\alpha(x)}, K^{\beta(x)}\right) .
\end{aligned}
$$

Following Schofield, we need to introduce the notion of the general rank of a morphism. For a morphism $\phi: V \rightarrow W$ between two representations of $Q$, define the rank function $\underline{\mathrm{rk}}(\phi) \in \mathbb{N}^{Q_{0}}$ by

$$
\underline{\mathrm{rk}}(\phi)(x)=\operatorname{dim}_{K} \phi(x)(V(x)), \quad x \in Q_{0} .
$$

Define the variety

$$
\begin{array}{r}
H(\alpha, \beta)=\left\{(V, W, \phi) \in \operatorname{Rep}(Q, \alpha) \times \operatorname{Rep}(Q, \beta) \times \operatorname{Hom}\left(K^{\alpha}, K^{\beta}\right) \mid\right. \\
\left.\forall a \in Q_{1} \phi(h a) V(a)=W(a) \phi(t a)\right\} .
\end{array}
$$

There is a natural projection $p: H(\alpha, \beta) \rightarrow \operatorname{Rep}(Q, \alpha) \times \operatorname{Rep}(Q, \beta)$ such that the fiber of $(V, W) \in$ $\operatorname{Rep}(Q, \alpha) \times \operatorname{Rep}(Q, \beta)$ can be identified with $\operatorname{Hom}_{Q}(V, W)$. Suppose that $Z \subseteq \operatorname{Rep}(Q, \alpha) \times \operatorname{Rep}(Q, \beta)$ is an irreducible closed subset. There exists an open dense subset $U \subseteq Z$ such $U$ is smooth and the fibers of $p^{-1}(U) \rightarrow U$ have constant dimension. Then $p^{-1}(U)$ is irreducible. The rank depends semi-continuously on $(V, W, \phi) \in p^{-1}(U)$. So there exists an open dense subset $U^{\prime} \subseteq p^{-1}(U)$ such that $\underline{\mathrm{rk}}(\phi)$ is constant on $U^{\prime}$. The value of this rank is called the general rank of a morphism $\phi: V \rightarrow W$ with $(V, W) \in Z$.

By symmetry, The Generalized Fulton Conjecture (Theorem 2.23) follows from

Theorem 7.16. If $\alpha \circ \beta=1$, then $\alpha \circ(n \beta)=1$ for all $n \geq 0$.

Proof. Assume that $\alpha \circ \beta=1$ and $\alpha \circ(n \beta)>1$ for some $n \geq 0$. Let us put $\sigma=-\langle\cdot, \beta\rangle$. Then

$$
\operatorname{dim} \operatorname{SI}(Q, \alpha)_{n \sigma}=\alpha \circ(n \beta) .
$$

We construct a quotient as in Section 2.8, Define the projective variety

$$
Y=\operatorname{Proj} \bigoplus_{n \geq 0} \operatorname{SI}(Q, \alpha)_{n \sigma}
$$

It has dimension $>0$. Let

$$
\pi: \operatorname{Rep}(Q, \alpha)_{\sigma}^{\mathrm{ss}} \rightarrow Y .
$$

be the GIT quotient with respect to $\sigma$, where $\operatorname{Rep}(Q, \alpha)_{\sigma}^{\text {ss }} \subseteq \operatorname{Rep}(Q, \alpha)$ is the dense, open subset of $\sigma$-semi-stable representations. Let $W \in \operatorname{Rep}(Q, \beta)$ in general position. The Schofield semi-invariant $c_{W} \in \operatorname{SI}(Q, \alpha)_{\sigma}$ is nonzero because $W$ is in general position. By assumption, $\operatorname{dim}_{K} \operatorname{SI}(Q, \alpha)_{\sigma}=\alpha \circ \beta=$ 1. $\operatorname{So} \operatorname{SI}(Q, \alpha)_{\sigma}$ is spanned by $c_{W}$. Also, if $W^{\prime} \in \operatorname{Rep}(Q, \beta)$ is any other representation then either $c_{W^{\prime}}$ is identically 0 or $c_{W^{\prime}}$ is equal to $c_{W}$ up to a scalar. By assumption, $\operatorname{dim} \operatorname{SI}(Q, \alpha)_{n \sigma}=\alpha \circ(n \beta)>1$ for some $n$. If $f_{1}, f_{2} \in \operatorname{SI}(Q, \alpha)_{n \sigma}$ are linearly independent, then they must be algebraically independent. This implies that the Krull dimension of the ring $\bigoplus_{n \geq 0} \operatorname{SI}(Q, \alpha)_{n \sigma}$ is at least 2, and the dimension of 
$Y$ is at least 1 . The equation $c_{W}=0$ defines a nonnegative divisor on the projective variety $Y$. Clearly this divisor is nonzero, because $c_{W}$ is not a constant. Let $y \in Y$ such that $C_{W}(y)=0$. Since $\pi$ is surjective, we can choose $V \in \pi^{-1}(y) \subseteq \operatorname{Rep}(Q, \alpha)_{\sigma}^{\text {ss }}$. It follows that $c_{W}(V)=0$. Let $D \subseteq \operatorname{Rep}(Q, \alpha)$ be an irreducible component of the divisor

$$
\left\{Z \in \operatorname{Rep}(Q, \alpha) \mid c_{W}(Z)=0\right\}
$$

which contains $V$. In conclusion, $D$ is a divisor of $\operatorname{Rep}(Q, \alpha)$ containing $\sigma$-semi-stable representations, and $c_{W}(V)=0$ for all $(V, W) \in D \times \operatorname{Rep}(Q, \beta)$.

Let $\gamma$ be the general rank of a homomorphism $\phi: V \rightarrow W$ where $(V, W) \in D \times \operatorname{Rep}(Q, \beta)$.

Define

$$
\begin{aligned}
\widetilde{M}=\left\{\left(V, W, V_{1}, W_{1}, \phi\right)\right. & \in \operatorname{Rep}(Q, \alpha) \times \operatorname{Rep}(Q, \beta) \times \operatorname{Gr}\left(\begin{array}{c}
\alpha \\
\alpha-\gamma
\end{array}\right) \times \operatorname{Gr}\left(\begin{array}{c}
\beta \\
\gamma
\end{array}\right) \times \operatorname{Hom}\left(K^{\alpha}, K^{\beta}\right) \mid \\
\phi & \left.: V \rightarrow W \text { is a morphism, } \forall x \in Q_{0} \phi\left(V_{1}(x)\right)=0 \text { and } \phi\left(K^{\alpha(x)}\right) \subseteq W_{1}(x)\right\} .
\end{aligned}
$$

and

$$
M=\left\{\left(V, W, V_{1}, W_{1}, \phi\right) \in \widetilde{M} \mid \phi\left(K^{\alpha(x)}\right)=W_{1}(x)\right\} .
$$

We have that $\widetilde{M}$ is a Zariski closed subset of

$$
\operatorname{Rep}(Q, \alpha) \times \operatorname{Rep}(Q, \beta) \times \operatorname{Gr}\left(\begin{array}{c}
\alpha \\
\alpha-\gamma
\end{array}\right) \times \operatorname{Gr}\left(\begin{array}{l}
\beta \\
\gamma
\end{array}\right) \times \operatorname{Hom}\left(K^{\alpha}, K^{\beta}\right),
$$

and $M$ is open in $\widetilde{M}$ and therefore it is locally closed. So $M$ is a variety. If $\left(V, W, V_{1}, W_{1}, \phi\right) \in M$ then $\phi: V \rightarrow W$ has kernel $V_{1}$, image $W_{1}$ and $\operatorname{rank} \gamma=\underline{\operatorname{dim}} W_{1}$.

Define

$$
\begin{aligned}
N:=\left\{\left(V_{1}, W_{1}, \phi\right) \in \operatorname{Gr}\left(\begin{array}{c}
\alpha \\
\alpha-\gamma
\end{array}\right) \times \operatorname{Gr}\left(\begin{array}{l}
\beta \\
\gamma
\end{array}\right) \times \operatorname{Hom}\left(K^{\alpha(x)}, K^{\beta(x)}\right) \mid\right. \\
\\
\left.\forall x \in Q_{0} \phi\left(V_{1}(x)\right)=0, \phi\left(K^{\alpha(x)}\right)=W_{1}(x)\right\} .
\end{aligned}
$$

Again $N$ is locally closed, hence a variety. The projection $p: M \rightarrow \operatorname{Gr}\left(\begin{array}{c}\alpha \\ \alpha-\gamma\end{array}\right) \times \operatorname{Gr}\left(\begin{array}{l}\beta \\ \gamma\end{array}\right)$ factors through a morphism $q: M \rightarrow N$ and the projection $r: N \rightarrow \operatorname{Gr}\left(\begin{array}{c}\alpha \\ \alpha-\gamma\end{array}\right) \times \operatorname{Gr}\left(\begin{array}{c}\beta \\ \gamma\end{array}\right)$.

Let $\left(V_{1}, W_{1}\right) \in \operatorname{Gr}\left(\begin{array}{c}\alpha \\ \alpha-\gamma\end{array}\right) \times \operatorname{Gr}\left(\begin{array}{l}\beta \\ \gamma\end{array}\right)$ and consider the fiber $r^{-1}\left(V_{1}, W_{1}\right)$. This can be seen as the set of all $\phi: K^{\alpha} / V_{1} \rightarrow W_{1}$ which induce an isomorphism at each vertex. So $r^{-1}\left(V_{1}, W_{1}\right) \cong \mathrm{GL}(Q, \gamma)$ has dimension

$$
\sum_{x \in Q_{0}} \gamma(x)^{2}
$$

Note that $r$ is actually a fiber bundle. We get

$$
\begin{aligned}
\operatorname{dim} N= & \operatorname{dim} \operatorname{Gr}\left(\begin{array}{c}
\alpha \\
\alpha-\gamma
\end{array}\right)+\operatorname{dim} \operatorname{Gr}\left(\begin{array}{l}
\beta \\
\gamma
\end{array}\right)+\sum_{x \in Q_{0}} \gamma(x)^{2}= \\
& =\sum_{x \in Q_{0}}\left(\gamma(x)(\alpha-\gamma)(x)+\gamma(x)(\beta-\gamma)(x)+\gamma(x)^{2}\right)=\sum_{x \in Q_{0}}(\gamma(x)(\alpha-\gamma)(x)+\gamma(x) \beta(x)) .
\end{aligned}
$$

The map $q: M \rightarrow N$ is a vector bundle. A fiber

$$
q^{-1}\left(V_{1}, W_{1}, \phi\right)
$$

is the set of all

$$
(V, W) \in \operatorname{Rep}(Q, \alpha) \times \operatorname{Rep}(Q, \beta)
$$


such that $V(a)\left(V_{1}(t a)\right) \subseteq V_{2}(h a)((\alpha-\gamma)(t a) \gamma(h a)$ linear constraints) for all $a$ and the restriction of $W(a)$ to $W_{1}(t a)$ is $\phi(h a) V(a) \phi(t a)^{-1}(\gamma(t a) \beta(h a)$ linear constraints) for all $a$. The dimension of $q^{-1}\left(V_{1}, W_{1}, \phi\right)$ is

$$
\operatorname{dim} \operatorname{Rep}(Q, \alpha)+\operatorname{dim} \operatorname{Rep}(Q, \beta)-\sum_{a \in Q_{1}}((\alpha-\gamma)(t a) \gamma(h a)-\gamma(t a) \beta(h a)) .
$$

Therefore,

$$
\begin{aligned}
& \operatorname{dim} M=\operatorname{dim} \text { fiber of } q+\operatorname{dim} N=\operatorname{dim} \operatorname{Rep}(Q, \alpha)+\operatorname{dim} \operatorname{Rep}(Q, \beta) \\
& -\sum_{a \in Q_{1}}((\alpha-\gamma)(t a) \gamma(h a)-\gamma(t a) \beta(h a))+\sum_{x \in Q_{0}}(\gamma(x)(\alpha-\gamma)(x)+\gamma(x) \beta(x))= \\
& \quad=\operatorname{dim} \operatorname{Rep}(Q, \alpha)+\operatorname{dim} \operatorname{Rep}(Q, \beta)-\langle\alpha-\gamma, \beta-\gamma\rangle .
\end{aligned}
$$

(Remember that $\langle\alpha, \beta\rangle=0)$. Since $M$ is a fiber bundle over the smooth irreducible variety $\operatorname{Gr}\left(\begin{array}{c}\alpha \\ \alpha-\gamma\end{array}\right) \times$ $\operatorname{Gr}\left(\begin{array}{l}\beta \\ \gamma\end{array}\right)$ with smooth irreducible fibers, we have that $M$ is smooth and irreducible.

Consider now the projection $s: M \rightarrow \operatorname{Rep}(Q, \alpha) \times \operatorname{Rep}(Q, \beta)$. Since $\gamma$ is the general rank of a homomorphism $\phi: V \rightarrow W$ for $(V, W) \in D \times \operatorname{Rep}(Q, \beta)$, we see that $\overline{s(M)}$ contains $D \times \operatorname{Rep}(Q, \beta)$. Since $\overline{s(M)}$ is irreducible and $D \times \operatorname{Rep}(Q, \beta)$ has codimension 1, we must have $\overline{s(M)}=D \times \operatorname{Rep}(Q, \beta)$ or $\overline{s(M)}=\operatorname{Rep}(Q, \alpha) \times \operatorname{Rep}(Q, \beta)$. The latter is impossible, because $\operatorname{hom}(\alpha, \beta)=0$ and $\gamma \neq 0$. Therefore $\overline{s(M)}=D \times \operatorname{Rep}(Q, \beta)$. The dimension of a general fiber of $s$ is

$$
\begin{aligned}
\operatorname{dim} M-\operatorname{dim} D \times \operatorname{Rep}(Q, \beta)=\operatorname{dim} M-\operatorname{dim} \operatorname{Rep}(Q, \alpha)-\operatorname{dim} \operatorname{Rep}(Q, \beta) & +1= \\
& =-\langle\alpha-\gamma, \beta-\gamma\rangle+1 .
\end{aligned}
$$

Choose $\left(V, W, V_{1}, W_{1}, \phi\right) \in M$ in general position. Then $(V, W) \in D \times \operatorname{Rep}(Q, \beta)$ is in general position. Then a general element in $\operatorname{Hom}_{Q}(V, W)$ has rank $\gamma$. The fiber $s^{-1}(V, W)$ is the set of all $\psi \in \operatorname{Hom}_{Q}(V, W)$ of rank $\gamma$. Therefore,

$$
\operatorname{dim} \operatorname{Ext}_{Q}(V, W)=\operatorname{dim} \operatorname{Hom}_{Q}(V, W)=\operatorname{dim} s^{-1}(V, W)=-\langle\alpha-\gamma, \beta-\gamma\rangle+1 .
$$

Suppose that $V_{1}^{\prime} \in \operatorname{Rep}(Q, \alpha-\gamma)$ and $W^{\prime} \in \operatorname{Rep}(Q, \beta)$ are both in general position. We know that a general representation of dimension $\beta$ has a $\gamma$-dimensional subrepresentation, because $\overline{s(M)}=$ $D \times \operatorname{Rep}(Q, \beta)$. So let $W_{1}^{\prime}$ be a $\gamma$-dimensional subrepresentation of $W^{\prime}$. Define $V^{\prime}=V_{1}^{\prime} \oplus W_{1}^{\prime}$ and let $\phi^{\prime}: V^{\prime} \rightarrow W^{\prime}$ be the projection $V^{\prime} \rightarrow W_{1}^{\prime} \subseteq W^{\prime}$. Now $\left(V^{\prime}, W^{\prime}, V_{1}^{\prime}, W_{1}^{\prime}, \phi^{\prime}\right) \in M$. Moreover $\operatorname{dim} \operatorname{Ext}\left(V_{1}^{\prime}, W^{\prime}\right)$ has the minimum possible value $\operatorname{ext}(\alpha-\gamma, \beta)$. The set of all $\left(V^{\prime}, W^{\prime}, V_{1}^{\prime}, W_{1}^{\prime}, \phi^{\prime}\right) \in M$ for which $\operatorname{dim} \operatorname{Ext}_{Q}\left(V_{1}^{\prime}, W^{\prime}\right)$ is minimal (or equivalently $\operatorname{dim} \operatorname{Hom}_{Q}\left(V_{1}^{\prime}, W^{\prime}\right)$ is minimal) is an open dense subset of $M$. Since $\left(V, W, V_{1}, W_{1}, \phi\right)$ was assumed to be in general position, we may assume $\operatorname{dim} \operatorname{Ext}_{Q}\left(V_{1}, W\right)=\operatorname{ext}(\alpha-\gamma, \beta)$.

From Theorem 2.19 follows that there exists a $\delta \hookrightarrow \alpha-\gamma$ such that

$$
\operatorname{ext}(\alpha-\gamma, \beta)=-\langle\delta, \beta\rangle
$$

Since $V \in D$ is in general position, we know that $V$ is $\sigma$-semi-stable. But $V$ has a $\delta$-dimensional subrepresentation $V_{2} \subseteq V_{1} \subseteq V$. Hence

$$
\operatorname{ext}(\alpha-\gamma, \beta)=-\langle\delta, \beta\rangle=\sigma(\delta) \leq 0
$$

by semi-stability. It follows that $\operatorname{ext}(\alpha-\gamma, \beta)=0$ and $\operatorname{Ext}_{Q}\left(V_{1}, W\right)=0$. From the long exact sequence of Ext's, the map $\operatorname{Ext}_{Q}\left(V_{1}, W\right) \rightarrow \operatorname{Ext}_{Q}\left(V_{1}, W / W_{1}\right)$ is surjective. So we also get $\operatorname{Ext}_{Q}\left(V_{1}, W / W_{1}\right)=0$. Now

$$
0=\operatorname{dim} \operatorname{Ext}_{Q}\left(V_{1}, W / W_{1}\right)=-\langle\alpha-\gamma, \beta-\gamma\rangle+\operatorname{dim} \operatorname{Hom}_{Q}\left(V_{1}, W / W_{1}\right)
$$


and so

$$
-\langle\alpha-\gamma, \beta-\gamma\rangle \leq 0
$$

On the other hand, $\operatorname{Hom}_{Q}(V, W) \neq 0$, so

$$
1 \leq \operatorname{dim} \operatorname{Hom}_{Q}(V, W)=\operatorname{dim}_{\operatorname{Ext}_{Q}}(V, W)=-\langle\alpha-\gamma, \beta-\gamma\rangle+1
$$

We conclude that

$$
-\langle\alpha-\gamma, \beta-\gamma\rangle=0 \text {. }
$$

From this follows that $\operatorname{Hom}_{Q}\left(V_{1}, W / W_{1}\right)=0$, by (22). We have an exact sequence

$$
\cdots \rightarrow \operatorname{Hom}_{Q}\left(V_{1}, W / W_{1}\right) \rightarrow \operatorname{Ext}_{Q}\left(V_{1}, W_{1}\right) \rightarrow \operatorname{Ext}_{Q}\left(V_{1}, W\right) \rightarrow \cdots
$$

Since the outer two are equal to 0 , we get $\operatorname{Ext}\left(V_{1}, W_{1}\right)=0$. This implies that $\operatorname{ext}(\alpha-\gamma, \gamma)=0$ and $(\alpha-\gamma) \hookrightarrow \alpha$. If $V^{\prime} \in \operatorname{Rep}(Q, \alpha) \backslash D$, then $V^{\prime}$ has an $(\alpha-\gamma)$-dimensional subrepresentation $V_{1}^{\prime}$. Define $W_{1}^{\prime}:=V^{\prime} / V_{1}^{\prime}$ and let $W_{2}^{\prime} \in \operatorname{Rep}(Q, \beta-\gamma)$ be any representation of dimension $\beta-\gamma$. Set $W^{\prime}=W_{1}^{\prime} \oplus W_{2}^{\prime}$

and let $\phi^{\prime}: V^{\prime} \rightarrow V^{\prime} / V_{1}^{\prime}=W_{1}^{\prime} \subseteq W^{\prime}$ be the projection. We have $\left(V^{\prime}, W^{\prime}, V_{1}^{\prime}, W_{1}^{\prime}, \phi^{\prime}\right) \in M$, so $\left(V^{\prime}, W^{\prime}\right) \in s(M)$ and $V^{\prime} \in D$. Contradiction!

\section{REFERENCES}

[1] S. Agnihotri, C. Woodward, Eigenvalues of products of unitary matrices and quantum Schubert calculus, Math. Res. Lett. 5 (1998), no. 6, 817-836.

[2] P. Belkale, Local systems on $\mathbb{P}^{1}-S$ for $S$ a finite set, Compositio Math. 129 (2001), no. 1, 67-86.

[3] P. Belkale, Geometric proofs of Horn and saturation conjectures, J. Algebraic Geom. 15 (2006), no. 1, $133-173$.

[4] P. Belkale, Geometric proof of a conjecture of Fulton, preprint.

[5] A. .S. Buch, The saturation conjecture (after A. Knutson and T. Tao), With an appendix by William Fulton, Enseign. Math. (2) 46 (2000), no. 1-2, 43-60.

[6] C. Chindris, Quivers, long exact sequences and Horn type inequalities, preprint, arXiv math.RT/0410423

[7] C. Chindris, Quivers, long exact sequences and Horn type inequalities II, preprint, 2004.

[8] C. Chindris, H. Derksen, J. Weyman, Non-log-concave Littlewood-Richardson coefficients, to appear in Comp. Math.

[9] W. Crawley-Boevey, Exceptional sequences of representations of quivers, Canadian Math. Soc. Conf. Proceedings, Vol 14 (1993), 117-124.

[10] W. Crawley-Boevey, Subrepresentations of general representations of quivers, Bull. London Math. Soc. 28 (1996), no. $4,363-366$.

[11] W. Crawley-Boevey, On matrices in prescribed conjugacy classes with no common invariant subspace and sum zero, Duke Math. J. 118 (2003), no. 2, 339-352.

[12] W. Crawley-Boevey, Ch. Geiss, Horn's problem and semi-stability for quiver representations, Representations of algebra. Vol. I, II, 40-48, Beijing Norm. Univ. Press, Beijing, 2002.

[13] H. Derksen, J. Weyman, Semi-invariants of quivers and saturation for Littlewood-Richardson coefficients, Journal of the AMS 13 (2000), 467-579.

[14] H. Derksen, J. Weyman, On the canonical decomposition of quiver representations, Compositio Math. 133 (2002), $245-265$.

[15] H. Derksen, J. Weyman, On the Littlewood-Richardson polynomials, J. Algebra 255 (2002), no. 2, $247-257$.

[16] H. Derksen, A. Schofield, J. Weyman, On the number of subrepresentations of a general quiver representation, to appear in J. London Math. Soc.

[17] S. Fomin, W. Fulton, C.-K. Li, Y.-T. Poon, Eigenvalues, singular values, and Littlewood-Richardson coefficients, Amer. J. Math. 127 (2005), no. 1, 101-127.

[18] W. Fulton, Eigenvalues of sums of Hermitian matrices (after A. Klyachko), Séminaire Bourbaki. Vol. $1997 / 98$. Astérisque No. 252, (1998), Exp. No. 845, 5, 255-269.

[19] W. Fulton, Eigenvalues, invariant factors, highest weights, and Schubert calculus, Bull. Amer. Math. Soc. 37, no. 3, 209-249.

[20] W. Fulton, Eigenvalues of majorized Hermitian matrices and Littlewood-Richardson coefficients, Special Issue: Workshop on Geometric and Combinatorial Methods in the Hermitian Sum Spectral Problem (Coimbra, 1999). Linear Algebra Appl. 319 (2000), no. 1-3, 23-36.

[21] A. Horn, Eigenvalues of sums of Hermitian matrices, Pacific J. Math. 12 (1962), 620-630.

[22] V. Kac, Infinite root systems, representations of graphs and Invariant Theory, Invent. Math. 56 (1980), 57-92.

[23] V. Kac, Infinite Root Systems, Representations of Graphs and Invariant Theory II, J. Algebra 78 (1982), 141-162. 
[24] A. D. King, Moduli of representations of finite dimensional algebras, Quart. J. Math. Oxford (2) 45 (1994), $515-530$.

[25] A. Kirillov, An invitation to the generalized saturation conjecture, Publ. Res. Inst. Math. Sci. 40 (2004), no. 4, $1147-1239$

[26] T. Klein, The multiplication of Schur-functions and extensions of p-modules, J. London Math. Soc. 43 (1968), 280-284.

[27] A. Klyachko, Stable bundles, representation theory and Hermitian operators, Selecta Math. (N.S.) 4 (1998), no. 3, 419-445.

[28] A. Knutson, T. Tao, The honeycomb model of $\mathrm{GL}_{n}(\mathbb{C})$ tensor products. I. Proof of the saturation conjecture, J. Amer. Math. Soc. 12 (1999), no. 4, 1055-1090.

[29] A. Knutson, T. Tao, C. Woodward, The honeycomb model of $\mathrm{GL}_{n}(\mathbb{C})$ tensor products. II. Puzzles determine facets of the Littlewood-Richardson cone, J. Amer. Math. Soc. 17 (2004), no. 1, 19-48.

[30] L. Le Bruyn, C. Procesi, Semisimple representations of quivers, Trans. Amer. Math. Soc. 317 (1990), no. 2, $585-598$.

[31] C.-K. Li, Y.-T. Poon, Off-diagonal submatrices of a Hermitian matrix, Proc. Amer. Math. Soc. 132 (2004), no. 10, $2849-2856$

[32] A. Okounkov, Why would multiplicities be log-concave?, The orbit method in geometry and physics (Marseille 2000), Progr. Math. 213, Birkhäuser Boston, MA, 2003,

[33] E. Rassart, A polynomiality property for Littlewood-Richardson coefficients, J. Combin. Theory Ser. A 107 (2004), no. $2,161-179$.

[34] A. Rudakov, Stability for an abelian category, J. Algebra 197 (1997), 231-245

[35] C. M. Ringel, Representations of K-species and bimodules, J. Algebra 41 (1976), 269-302.

[36] C. M. Ringel, Tame algebras and integral quadratic forms, Lecture Notes in Math. 1099, Springer, 1984.

[37] C. M. Ringel, The braid group action on the set of exceptional sequences of a hereditary Artin algebra, Abelian group theory and related topics (Oberwolfach, 1993), Contemp. Math. 171, Amer. Math. Soc., Providence, RI, 1994, 339-352.

[38] A. Schofield, Semi-invariants of Quivers, J. London Math. Soc. 43 (1991), 383-395.

[39] A. Schofield, General Representations of Quivers, Proc. London Math. Soc. (3) 65 (1992), 46-64.

[40] A. Schofield, Birational classification of moduli spaces of representations of quivers, Indag. Math., N.S. 12 (3), 407-432.

[41] A. Schofield, M. van den Bergh, Semi-invariants of quivers for arbitrary dimension vectors, Indag. Mathem., N.S. 12(1), 125-138.

[42] R. Vakil, Schubert Induction, to appear in Ann. of Math., arXiv:math.AG/0309227

[43] H. Weyl, Das asymtotische Verteilungsgesetz der Eigenwerte lineare partieller Differentialgleichungen, Math. Ann. 71 (1912), 441-479.

[44] C. Woodward, Gromov-Witten invariants of flag manifolds and products of conjugacy classes, Advances in algebraic geometry motivated by physics (Lowell, MA, 2000), 279-286, Contemp. Math., 276, AMS, 2001. 